\title{
Massive neutrinos and cosmology
}

\author{
Julien Lesgourgues ${ }^{a}$ and Sergio Pastor ${ }^{b}$ \\ ${ }^{a}$ Laboratoire de Physique Théorique LAPTH (CNRS-Université de Savoie) \\ B.P. 110, F-74941 Annecy-le-Vieux Cedex, France \\ ${ }^{\mathrm{b}}$ Instituto de Física Corpuscular (CSIC-Universitat de València) \\ Ed. Institutos de Investigación, Apdo. 22085, E-46071 Valencia, Spain
}

\begin{abstract}
The present experimental results on neutrino flavour oscillations provide evidence for non-zero neutrino masses, but give no hint on their absolute mass scale, which is the target of beta decay and neutrinoless double-beta decay experiments. Crucial complementary information on neutrino masses can be obtained from the analysis of data on cosmological observables, such as the anisotropies of the cosmic microwave background or the distribution of large-scale structure. In this review we describe in detail how free-streaming massive neutrinos affect the evolution of cosmological perturbations. We summarize the current bounds on the sum of neutrino masses that can be derived from various combinations of cosmological data, including the most recent analysis by the WMAP team. We also discuss how future cosmological experiments are expected to be sensitive to neutrino masses well into the sub-eV range.
\end{abstract}

Key words: Neutrino masses, Cosmology, Dark matter

PACS: 14.60.Pq, 95.35.+d, 98.80.Es

\section{Contents}

$1 \quad$ Introduction 3

$2 \quad$ Neutrino oscillations and absolute neutrino mass searches 5

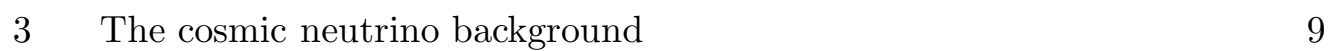

3.1 Basics on relic neutrinos, including neutrino decoupling 9

$\begin{array}{lll}3.2 & \text { Extra radiation and the effective number of neutrinos } & 13\end{array}$

Email addresses: lesgourg@lapp.in2p3.fr (Julien Lesgourgues), pastor@ific.uv.es (Sergio Pastor).

Preprint submitted to Elsevier Science 3 February 2008 
$4 \quad$ Massive neutrinos and cosmological perturbations 16

4.1 Observables targets: definition of the power spectra 18

\begin{tabular}{lll}
\hline 4.2 & Background evolution & 20
\end{tabular}

4.3 Gauge transformations and Einstein equations 21

4.4 Linear perturbation theorv in a neutrinoless Universe (pure $\Lambda$ CDM) 25

4.5 Linear perturbation theorv in presence of neutrinos ( $\Lambda \mathrm{MDM}) \quad 38$

$\begin{array}{lll}4.6 & \text { Summary of the neutrino mass effects } & 60\end{array}$

\begin{tabular}{|ll}
5 & Current observations and bounds
\end{tabular}

5.1 Statistical methods for parameter inference from cosmological data 67

$\begin{array}{lll}5.2 & \text { CMB anisotropies } & 71\end{array}$

\begin{tabular}{lll}
\hline 5.3 & Galaxv redshift survevs & 73
\end{tabular}

$\begin{array}{lll}5.4 & \text { Lvman- } \alpha \text { forest } & 76\end{array}$

\begin{tabular}{lll}
\hline 5.5 & Summary of current bounds & 77
\end{tabular}

\begin{tabular}{lll}
\hline 5.6 & Extra parameters & 79
\end{tabular}

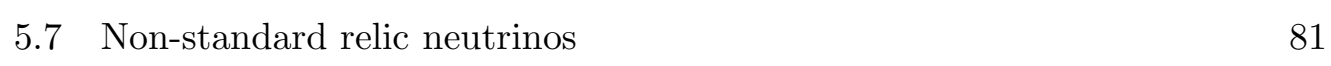

$6 \quad$ Future sensitivities and new experimental techniques 82

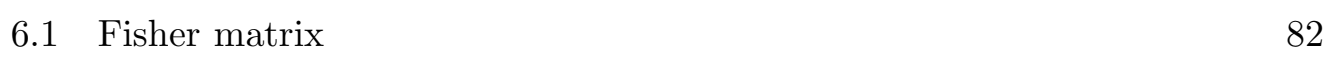

\begin{tabular}{|ll}
6.2 & Future CMB experiments
\end{tabular}

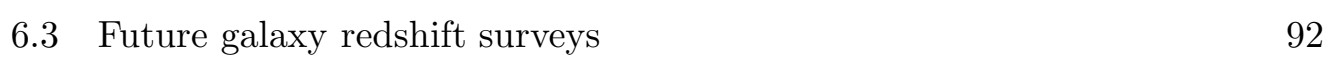

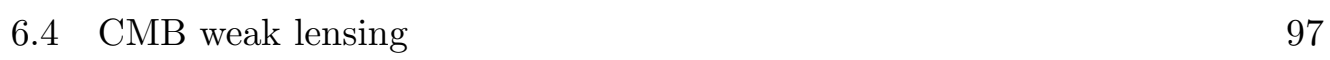

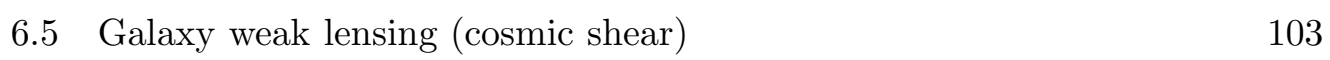

\begin{tabular}{lll}
\hline 6.6 & Galaxv cluster survevs & 107
\end{tabular}

\begin{tabular}{lll}
\hline Conclusions & 108
\end{tabular}

$\begin{array}{lr}\text { References } & 111\end{array}$ 


\section{Introduction}

Neutrino cosmology is a fascinating example of the fecund interaction between particle physics and astrophysics. At the present time, the researchers working on neutrino physics know that the advances in this field need a combined effort in the two areas.

From the point of view of cosmologists, the idea that massive neutrinos could play a significant role in the history of the Universe and in the formation of structures has been discussed for more than thirty years, first as a pure speculation. However, nowadays we know from experimental results on flavour neutrino oscillations that neutrinos are massive. At least two neutrino states have a large enough mass for being non-relativistic today, thus making up a small fraction of the dark matter of the Universe. At a stage in which cosmology reaches high precision thanks to the large amount of observational data, it is unavoidable to take into account the presence of massive neutrinos. In particular, the observable matter density power spectrum is damped on small scales by massive neutrinos. This effect can range from a few per cent for $0.05-0.1 \mathrm{eV}$ masses, the minimal values of the total neutrino mass compatible with oscillation data, up to $10-20 \%$ in the limit of three degenerate masses.

From the point of view of particle physicists, fixing the absolute neutrino mass scale (or, equivalently, the lightest neutrino mass once the data on flavour oscillations are taken into account) is the target of terrestrial experiments such as the searches for neutrinoless double beta decay or tritium beta decay experiments, a difficult task in spite of huge efforts and very promising scheduled experiments. At the moment, the best bounds come from the analysis of cosmological data, from the requirement that neutrinos did not wash out too much of the small-scale cosmological structures. Recently the cosmological limits on neutrino masses progressed in a spectacular way, in particular thanks to the precise observation of the Cosmic Microwave Background (CMB) anisotropies by the Wilkinson Microwave Anisotropy Probe (WMAP) satellite, and to the results of a new generation of very deep galaxy redshift surveys. Neutrino physicists look with anxiety at any new development in this field, since in the next years many cosmological observations will be available with unprecedented precision.

At this very exciting moment, which might be preceding a breaking discovery within a few years, our aim is to present here a clear and comprehensive review of the role of massive neutrinos in cosmology, including a description of the underlying theory of cosmological perturbations, a summary of the current bounds and a review of the sensitivities expected for future cosmological observations. Our main goal is to address this manuscript simultaneously to 
particle physicists and cosmologists: for each aspect, we try to avoid useless jargon and to present a self-contained summary. Compared to recent short reviews on the subject, such as Refs. $[1,2,3,4]$, we tried to present a more detailed discussion. At the same time, we will focus on the case of three flavour neutrinos with masses in accordance with the current non-cosmological data. A recent review on primordial neutrinos can be found in [5], while for many other aspects of neutrino cosmology we refer the reader to [6]. Finally, a more general review on the connection between particle physics and cosmology can be found in [7].

We begin in Sec. 2 with an introduction to flavour neutrino oscillations, and their implications for neutrino masses in the standard three-neutrino scenario. We also briefly review the limits on neutrino masses from laboratory experiments. Then, in Sec. 3, we describe some basic properties of the Cosmic Neutrino Background. According to the Big Bang cosmological model, each of the three flavour neutrinos were in thermal equilibrium in the Early universe, and then decoupled while still relativistic. We review the consequences of this simple assumption for the phase-space distribution and number density of the relic neutrinos, and we summarize how this standard picture is confirmed by observations from Big Bang Nucleosynthesis (BBN) and cosmological perturbations. Finally we explain why neutrinos are expected to constitute a fraction of the dark matter today.

In Sec. 4, we describe the impact of massive neutrinos on cosmological perturbations. This section is crucial for understanding the rest, since current and future bounds rely precisely on the observation of cosmological perturbations. Although the theory of cosmological perturbations is a very vast and technical topic, we try here to be accessible both to particle physicists willing to learn the field, and to cosmologists willing to understand better the specific role of neutrinos. We introduce the minimal number of concepts, technicalities and equations for understanding the main results related to neutrinos. Still this section is quite long because we wanted to make it self-contained, and to explain many intermediate steps that are usually hidden in most works on the subject.

In Sec. 4.1, we define the quantities that can be observed, i.e. those that we want to compute. In Sec. 4.2, we describe the evolution of homogeneous quantities and in Sec. 4.3 we define the general setup for the theory of cosmological perturbations. Then in Sec. 4.4 we described in a rather simplified way what the evolution of perturbations would look like in absence of neutrinos. For a cosmologist, these four sections are part of common knowledge and can be skipped. In Sec. 4.5, we present a detailed description of the impact of neutrinos on cosmological perturbations. This long section is the most technical part of this work, so the reader who wants to avoid technicalities and to know the results can go directly to Sec. 4.6, for a comprehensive summary of the 
effects of neutrino masses on cosmological observables.

Then, in Sec. 5 we review the current existing bounds on neutrino masses, starting from those involving only CMB observations, and then adding different sets of data on the distribution of the Large Scale Structure (LSS) of the Universe, which include those coming from galaxy redshift surveys and the Lyman- $\alpha$ forest. We explain why a unique cosmological bound on neutrino masses does not exist, and why there are significant variations from paper to paper, depending on the data included and the assumed cosmological model.

Finally, in Sec. 6 we describe the prospects for the next ten or fifteen years. We summarize the forecasts which can be found in the literature concerning the sensitivity to neutrino masses of future CMB experiments, galaxy redshift surveys, weak lensing observations (either from the CMB or from galaxy ellipticity) and galaxy cluster surveys. We conclude with some general remarks in Sec. 7.

\section{Neutrino oscillations and absolute neutrino mass searches}

Neutrinos have played a fundamental role in the understanding of weak interactions since they were postulated by W. Pauli in 1930 to safeguard energy conservation in beta decay processes. These chargeless leptons are massless in the framework of the succesful Standard Model (SM) of particle physics. However, this is an accidental prediction of the SM, and there are many wellmotivated extended models where neutrinos acquire mass and other non-trivial properties (see e.g. $[8,9,10,11]$ ). Thus the measurement of neutrino masses could give us some hints on the new fundamental theory, of which the SM is just the low-energy limit, and in particular on new energy scales.

It was realized by B. Pontecorvo in 1957 that if neutrinos were massive there could exist processes where the neutrino flavour is not conserved, that we call neutrino oscillations. For small neutrino masses, these oscillations actually take place on macroscopic distances and can be measured if we are able to detect neutrinos from distant sources, identify their flavour and compare the results with the theoretical predictions for the initial neutrino fluxes. Actually, after decades of experimental efforts in underground facilities, neutrinos have been detected from various natural or artificial sources. The former include the neutrinos produced in the nuclear reactions in the Sun, as secondary particles from the interaction of cosmic rays in the atmosphere of the Earth and even a few neutrinos were detected from a supernova explosion (SN1987A). We have also measured the neutrino fluxes originated in artificial sources such as nuclear reactors or accelerators. 


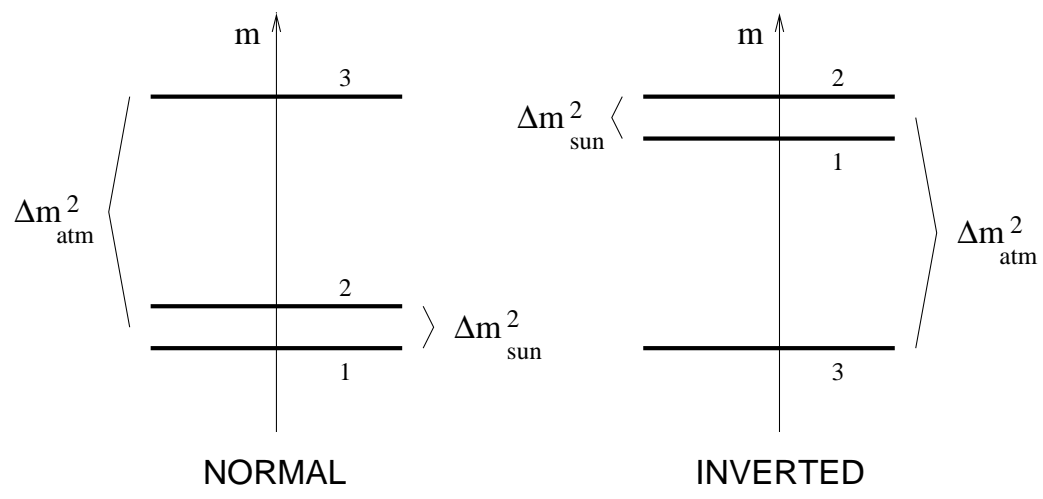

Fig. 1. The two neutrino schemes allowed if $\Delta m_{\mathrm{atm}}^{2} \gg \Delta m_{\text {sun }}^{2}$ : normal hierarchy $(\mathrm{NH})$ and inverted hierarchy $(\mathrm{IH})$.

Nowadays there exist compelling evidences for flavour neutrino oscillations from a variety of experimental data on solar, atmospheric, reactor and accelerator neutrinos. These are very important results, because the existence of flavour change implies that neutrinos mix and have non-zero masses, which in turn requires particle physics beyond the SM. There are many excellent reviews on neutrino oscillations and their implications, to which we refer the reader for more details (see e.g. the recent ones $[13,14]$ ).

We know that the number of light neutrinos sensitive to weak interactions (flavour or active neutrinos) equals three from the analysis of the invisible $Z$ boson width at LEP, $N_{\nu}=2.994 \pm 0.012$ [12], and the three flavour neutrinos $\left(\nu_{e}, \nu_{\mu}, \nu_{\tau}\right)$ are linear combinations of states with definite mass $\nu_{i}$, where $i$ is the number of massive neutrinos.

In a three-neutrino scenario flavour and mass eigenstates are related by the mixing matrix $U$, parametrized as $[12,14]$

$$
\left(\begin{array}{ccc}
c_{12} c_{13} & s_{12} c_{13} & s_{13} e^{-i \delta} \\
-s_{12} c_{23}-c_{12} s_{23} s_{13} e^{i \delta} & c_{12} c_{23}-s_{12} s_{23} s_{13} e^{i \delta} & s_{23} c_{13} \\
s_{12} s_{23}-c_{12} c_{23} s_{13} e^{i \delta} & -c_{12} s_{23}-s_{12} c_{23} s_{13} e^{i \delta} & c_{23} c_{13}
\end{array}\right) .
$$

Here $c_{i j}=\cos \theta_{i j}$ and $s_{i j}=\sin \theta_{i j}$ for $i j=12,23$ or 13 , and $\delta$ is a CP-violating phase. Together with the three masses, in total there are seven flavour parameters in the neutrino sector. This would be all if neutrinos were Dirac particles, like the charged leptons, and the total lepton number is conserved. Instead, if neutrinos are Majorana particles (i.e. a neutrino is its own antiparticle), the matrix $U$ is multiplied by a diagonal matrix of phases that can be taken as $\operatorname{diag}\left(1, e^{i \phi_{2} / 2}, e^{i\left(\phi_{3}+2 \delta\right) / 2}\right)$. These two phases do not show up in neutrino oscillations, but appear in lepton-number-violating processes such as neutrinoless double beta decay, as discussed later. 

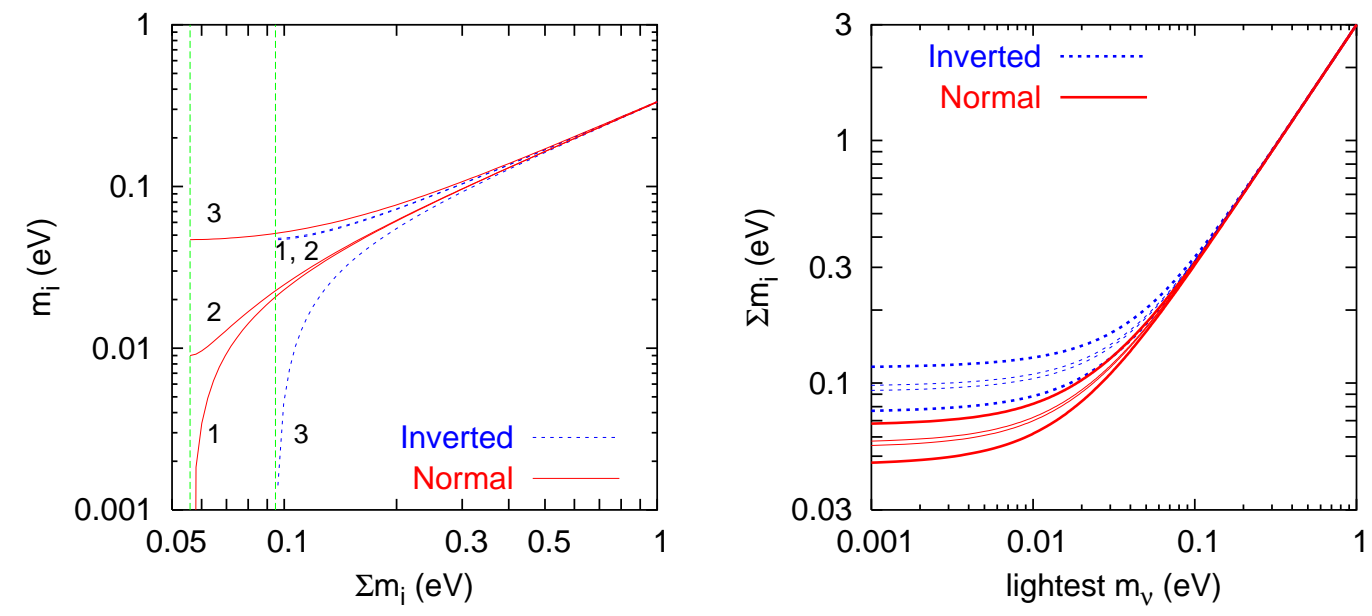

Fig. 2. Expected values of neutrino masses according to the values in Eq. (2). Left: individual neutrino masses as a function of the total mass for the best-fit values of the $\Delta m^{2}$. Right: ranges of total neutrino mass as a function of the lightest state within the $3 \sigma$ regions (thick lines) and for a future determination at the $5 \%$ level (thin lines).

Oscillation experiments can measure the differences of squared neutrino masses $\Delta m_{21}^{2}=m_{2}^{2}-m_{1}^{2}$ and $\Delta m_{31}^{2}=m_{3}^{2}-m_{1}^{2}$, the relevant ones for solar and atmospheric neutrinos, respectively. As a reference, we take the following $3 \sigma$ ranges of mixing parameters from an update of ref. [13],

$$
\begin{aligned}
\Delta m_{21}^{2} & =\left(7.9_{-0.8}^{+1.0}\right) \times 10^{-5} \mathrm{eV}^{2} \quad\left|\Delta m_{31}^{2}\right|=\left(2.2_{-0.8}^{+1.1}\right) \times 10^{-3} \mathrm{eV}^{2} \\
s_{12}^{2} & =0.30_{-0.06}^{+0.10} \quad s_{23}^{2}=0.50_{-0.16}^{+0.18} \quad s_{13}^{2} \leq 0.043
\end{aligned}
$$

Unfortunately oscillation experiments are insensitive to the absolute scale of neutrino masses, since the knowledge of $\Delta m_{21}^{2}>0$ and $\left|\Delta m_{31}^{2}\right|$ leads to the two possible schemes shown in Fig. 1, but leaves one neutrino mass unconstrained (see e.g. the discussion in the reviews $[14,15,16,17,18]$ ). These two schemes are known as normal (NH) and inverted (IH) hierarchies, characterized by the sign of $\Delta m_{31}^{2}$, positive and negative, respectively. For small values of the lightest neutrino mass $m_{0}$, i.e. $m_{1}\left(m_{3}\right)$ for $\mathrm{NH}(\mathrm{IH})$, the mass states follow a hierarchical scenario, while for masses much larger than the differences all neutrinos share in practice the same mass and then we say that they are degenerate. In general, the relation between the individual masses and the total neutrino mass can be found numerically, as shown in Fig. 2.

It is also possible that the number of massive neutrino states is larger than the number of flavor neutrinos. In such a case, in order to not violate the LEP results the extra neutrino states must be sterile, i.e. singlets of the SM gauge group and thus insensitive to weak interactions. At present, the results of the Liquid Scintillator Neutrino Detector (LSND) [19], an experiment that 
has measured the appearance of electron antineutrinos in a muon antineutrino beam, constitute an independent evidence of neutrino conversions at a larger mass difference than those in Eq. 2. In such a case, a fourth sterile neutrino is required with mass of $\mathcal{O}(\mathrm{eV})$ [13]. The LSND results will be checked by the ongoing MiniBoone experiment [20], whose first data are expected for 2006. In this review, we will mainly consider the three-neutrino scenario, but we briefly comment on the cosmological bounds on the four-neutrino mass schemes that include the LSND results in Sec. 5.6.

As we discuss in the next sections, cosmology is at first order sensitive to the total neutrino mass if all states have the same number density, providing information on $m_{0}$ but blind to neutrino mixing angles or possible CP violating phases. Thus cosmological results are complementary to terrestrial experiments such as beta decay and neutrinoless double beta decay, which are respectively sensitive to the effective masses

$$
\begin{aligned}
m_{\beta} & =\left(\sum_{i}\left|U_{e i}\right|^{2} m_{i}^{2}\right)^{1 / 2}=\left(c_{12}^{2} c_{13}^{2} m_{1}^{2}+s_{12}^{2} c_{13}^{2} m_{2}^{2}+s_{13}^{2} m_{3}^{2}\right)^{1 / 2}, \\
m_{\beta \beta} & =\left|\sum_{i} U_{e i}^{2} m_{i}\right|=\left|c_{12}^{2} c_{13}^{2} m_{1}+s_{12}^{2} c_{13}^{2} m_{2} e^{i \phi_{2}}+s_{13}^{2} m_{3} e^{i \phi_{3}}\right| .
\end{aligned}
$$

The search for a signal of non-zero neutrino masses in a beta decay experiment is, in principle, the best strategy for measuring directly the neutrino mass, since it involves only the kinematics of electrons (see [21] for a recent review). The current limits from tritium beta decay apply only to the range of degenerate neutrino masses, so that $m_{\beta} \simeq m_{0}$ (if unitarity of $U$ is assumed). The bound at 95\% CL is $m_{0}<2.05-2.3 \mathrm{eV}$ from the Troitsk [22] and Mainz [23] experiments, respectively. This value is expected to be improved by the KATRIN project [24] to reach a discovery potential for $0.3-0.35 \mathrm{eV}$ masses (or a sensitivity of $0.2 \mathrm{eV}$ at $90 \% \mathrm{CL}$ ).

The neutrinoless double beta decay $(Z, A) \rightarrow(Z+2, A)+2 e^{-}$(in short $\left.0 \nu 2 \beta\right)$ is a rare nuclear processes where lepton number is violated and whose observation would mean that neutrinos are Majorana particles. If the $0 \nu 2 \beta$ process is mediated by a light neutrino, the results from neutrinoless double beta decay experiments are converted into an upper bound or a measurement of the effective mass $m_{\beta \beta}$ in Eq. (3). A recent work [25] gives the following upper bounds at $99 \% \mathrm{CL}$

$$
\left|m_{\beta \beta}\right|<(0.44-0.62) h_{N} \mathrm{eV}
$$

where $h_{N}$ is a parameter that characterizes the uncertainties on the corresponding nuclear matrix elements (see [26] for a recent discussion and [15] 
for a review). The above range corresponds to the results of recent double beta decay experiments based on ${ }^{76} \mathrm{Ge}$, such as Heidelberg-Moscow [27] and IGEX [28], or ${ }^{130} \mathrm{Te}$ such as Cuoricino [29] (see e.g. [30] for results from other isotopes). In addition, there exists a claim of a positive $0 \nu 2 \beta$ signal which would correspond to the approximate range $0.1<\left|m_{\beta \beta}\right| / \mathrm{eV}<0.9[31]$. Future $0 \nu 2 \beta$ projects will improve the current sensitivities down to values of the order $\left|m_{\beta \beta}\right| \sim 0.01-0.05 \mathrm{eV}$ [15]. An experimental detection of $0 \nu 2 \beta$, in combination with results from beta decay or cosmology, will also help us to discriminate between the two neutrino mass spectra and to pin down the values of the neutrino flavour parameters, as discussed for instance in $[32,33]$.

There are other ways to obtain information on the absolute scale of neutrino masses, such as the measurement of the time-of-flight dispersion of a supernova neutrino signal (at most sensitive to masses of order some $\mathrm{eV}$, see [34] and references therein), but to conclude this section let us note that the sum of neutrino masses is restricted to the approximate range

$$
0.056(0.095) \mathrm{eV} \lesssim \sum_{i} m_{i} \lesssim 6 \mathrm{eV}
$$

where the upper limit comes exclusively from tritium beta decay results and the lower limit reflects the minimum values of the total neutrino mass in the normal (inverted) hierarchy.

\section{The cosmic neutrino background}

\subsection{Basics on relic neutrinos, including neutrino decoupling}

The existence of a relic sea of neutrinos is a generic prediction of the standard hot big bang model, in number only slightly below that of relic photons that constitute the CMB. Produced at large temperatures by frequent weak interactions, cosmic neutrinos were kept in equilibrium until these processes became ineffective in the course of the expansion. While coupled to the rest of the primeval plasma, neutrinos had a momentum spectrum with an equilibrium Fermi-Dirac form with temperature $T$,

$$
f_{\mathrm{eq}}(p)=\left[\exp \left(\frac{p-\mu_{\nu}}{T}\right)+1\right]^{-1} .
$$

Here we have included a neutrino chemical potential $\mu_{\nu}$ that would exist in the presence of a neutrino-antineutrino asymmetry, but it was shown in $[35,36,37]$ that the stringent $\mathrm{BBN}$ bounds on $\mu_{\nu_{e}}$ apply to all flavours, since neutrino 
oscillations lead to approximate flavour equilibrium before BBN. The current bounds on the common value of the neutrino degeneracy parameter $\xi_{\nu} \equiv \mu_{\nu} / T$ are $-0.05<\xi_{\nu}<0.07$ at $2 \sigma[38]$ (relaxed to $-0.13<\xi_{\nu}<0.3$ in presence of extra relativistic degrees of freedom $[39,40])$. Thus the contribution of a relic neutrino asymmetry can be safely ignored.

As the universe cools, the weak interaction rate $\Gamma_{\nu}$ falls below the expansion rate given by the Hubble parameter $H$ and one says that neutrinos decouple from the rest of the plasma. An estimate of the decoupling temperature can be found by equating the thermally averaged value of the weak interaction rate

$$
\Gamma_{\nu}=\left\langle\sigma_{\nu} n_{\nu}\right\rangle
$$

where $\sigma_{\nu} \propto G_{F}^{2}$ is the cross section of the electron-neutrino processes with $G_{F}$ the Fermi constant and $n_{\nu}$ is the neutrino number density, with the expansion rate

$$
H=\sqrt{\frac{8 \pi \rho}{3 M_{P}^{2}}},
$$

where $\rho$ is the total energy density and $M_{P}$ is the Planck mass. If we approximate the numerical factors to unity, with $\Gamma_{\nu} \approx G_{F}^{2} T^{5}$ and $H \approx T^{2} / M_{P}$, we obtain the rough estimate $T_{\mathrm{dec}} \approx 1 \mathrm{MeV}$. More accurate calculations give slightly higher values of $T_{\mathrm{dec}}$ which are flavour dependent since electron neutrinos and antineutrinos are in closer contact with $e^{ \pm}$, as shown e.g. in [6].

Although neutrino decoupling is not described by a unique $T_{\text {dec }}$, it can be approximated as an instantaneous process. The standard picture of instantaneous neutrino decoupling is very simple (see e.g. [41,42]) and reasonably accurate. In this approximation, the spectrum in Eq. (6) is preserved after decoupling, since both neutrino momenta and temperature redshift identically with the universe expansion. In other words, the number density of non-interacting neutrinos remains constant in a comoving volume since the decoupling epoch.

We have seen in Sec. 2 that active neutrinos cannot possess masses much larger than $1 \mathrm{eV}$, so they were ultra-relativistic at decoupling. This is the reason why the momentum distribution in Eq. (6) does not depend on the neutrino masses, even after decoupling, i.e. there is no neutrino energy in the exponential of $f_{\text {eq }}(p)$.

When calculating quantities related to relic neutrinos, one must consider the various possible degrees of freedom per flavour. If neutrinos are massless or Majorana particles, there are two degrees of freedom for each flavor, one for 
neutrinos (one negative helicity state) and one for antineutrinos (one positive helicity state). Instead, for Dirac neutrinos there are in principle twice more degrees of freedom, corresponding to the two helicity states. However, the extra degrees of freedom should be included in the computation only if they are populated and brought into equilibrium before the time of neutrino decoupling. In practice, the Dirac neutrinos with the "wrong-helicity" states do not interact with the plasma at temperatures of the $\mathrm{MeV}$ order and have a vanishingly small density with respect to the usual left-handed neutrinos (unless neutrinos have masses close to the keV range, as explained in Sec. 6.4 of [6], but such a large mass is excluded for active neutrinos). Thus the relic density of active neutrinos does not depend on their nature, either Dirac or Majorana particles.

Shortly after neutrino decoupling the photon temperature drops below the electron mass, favouring $e^{ \pm}$annihilations that heat the photons. If one assumes that this entropy transfer did not affect the neutrinos because they were already completely decoupled, it is easy to calculate the ratio between the temperatures of relic photons and neutrinos $T_{\gamma} / T_{\nu}=(11 / 4)^{1 / 3} \simeq 1.40102$. Any quantity related to relic neutrinos can be calculated at later times with the spectrum in Eq. (6) and $T_{\nu}$. For instance, the number density per flavour is fixed by the temperature,

$$
n_{\nu}=\frac{3}{11} n_{\gamma}=\frac{6 \zeta(3)}{11 \pi^{2}} T_{\gamma}^{3}
$$

which leads to a present value of 113 neutrinos and antineutrinos of each flavour per $\mathrm{cm}^{3}$. Instead, the energy density for massive neutrinos should in principle be calculated numerically, with two well-defined analytical limits,

$$
\begin{aligned}
& \rho_{\nu}\left(m_{\nu} \ll T_{\nu}\right)=\frac{7 \pi^{2}}{120}\left(\frac{4}{11}\right)^{4 / 3} T_{\gamma}^{4} \\
& \rho_{\nu}\left(m_{\nu} \gg T_{\nu}\right)=m_{\nu} n_{\nu}
\end{aligned}
$$

Thus we see that the contribution of massive neutrinos to the energy density in the non-relativistic limit is a function of the mass (or the sum of masses if all neutrino states have $m_{i} \gg T_{\nu}$ ).

In a more accurate analysis of neutrino decoupling, the standard picture described above is modified: the processes of neutrino decoupling and $e^{ \pm}$annihilations are sufficiently close in time so that some relic interactions between $e^{ \pm}$and neutrinos exist. These relic processes are more efficient for larger neutrino energies, leading to non-thermal distortions in the neutrino spectra and a slightly smaller increase of the comoving photon temperature, as noted in a series of works (see the full list given in the review [6]). A proper calculation of the process of non-instantaneous neutrino decoupling 

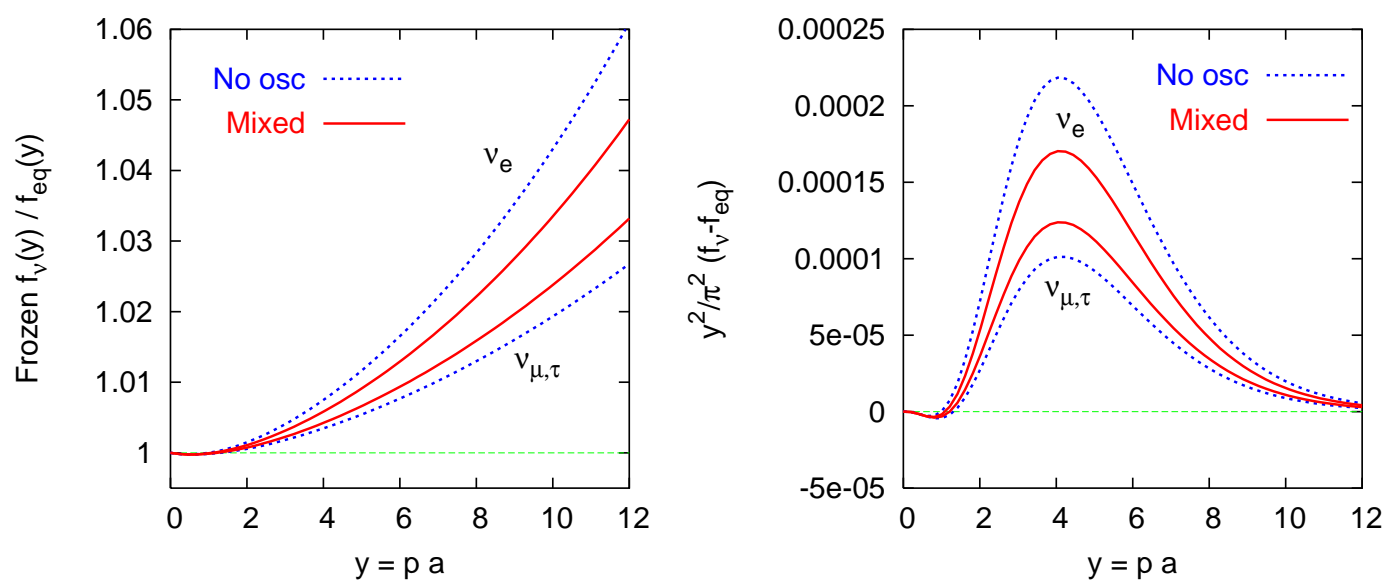

Fig. 3. The upper (lower) lines are the frozen distortions of the electron (muon or tau) neutrino spectra as a function of the comoving momentum $y$, calculated with (solid) and without (dotted) the effect of flavour oscillations. Left: real neutrino distribution functions normalized to the equilibrium one. Right: contribution of the distortions to the comoving number density. Here the scale factor was normalized so that $a(t) \rightarrow 1 / T_{\gamma}$ at large temperatures.

demands solving the Boltzmann equations for the neutrino spectra, a set of integro-differential kinetic equations that are difficult to solve numerically. The momentum-dependent calculations were carried out in refs. [43,44,45], while the inclusion of finite temperature QED corrections to the electromagnetic plasma was done in [46].

A recent work [47] has considered the effect of flavour neutrino oscillations on the neutrino decoupling process (see also [48]). The frozen values of the neutrino distributions are shown in Fig. 3, for the cases calculated with and without the effect of flavour oscillations. One can see that the distortions grow with the neutrino momentum, and their contribution to the number density of neutrinos is maximal around $y=4$. For the best-fit values of the mixing parameters in Eq. (2), Ref. [47] found an increase in the neutrino energy densities of $0.73 \%$ and $0.52 \%$ for $\nu_{e}$ 's and $\nu_{\mu, \tau}$ 's, respectively. At the same time, after $e^{ \pm}$annihilations the comoving photon temperature is a factor 1.3978 larger, instead of 1.40102 in the approximation of instantaneous decoupling. Note, however, that for any cosmological epoch when neutrino masses can be relevant one should consider the spectra of the neutrino mass eigenstates $\nu_{1,2,3}$ instead of flavour eigenstates $\nu_{e, \mu, \tau}$. In principle, for numerical calculations of the cosmological perturbation evolution such as those done by the Boltzmann codes CMBFAST [49] or CAMB [50], one should include the full distribution function of each mass eigenstate, including the distortions shown in Fig. 3. It is only when the neutrinos are relativistic or when one is interested in an observable that does not depend on neutrino masses (such as the number density) that the effect of the distortion can be simply integrated over momentum. 
For instance, the contribution of relativistic relic neutrinos to the total energy density is taken into account just by using $N_{\text {eff }}=3.046$ as defined later in Eq. (11). In practice, the distortions calculated in Ref. [47] only have small consequences on the evolution of cosmological perturbations, and for many purposes they can be safely neglected.

\subsection{Extra radiation and the effective number of neutrinos}

Neutrinos fix the expansion rate during the cosmological era when the Universe is dominated by radiation. Their contribution to the total radiation content can be parametrized in terms of the effective number of neutrinos $N_{\text {eff }}[51,52]$, through the relation

$$
\rho_{\mathrm{R}}=\left[1+\frac{7}{8}\left(\frac{4}{11}\right)^{4 / 3} N_{\mathrm{eff}}\right] \rho_{\gamma},
$$

where $\rho_{\gamma}$ is the energy density of photons, whose value today is known from the measurement of the CMB temperature. This equation is valid when neutrino decoupling is complete and holds as long as all neutrinos are relativistic.

In Sec. 2 we saw that from accelerator data the number of active neutrinos is three, while in the previous subsection we learned from the analysis of neutrino decoupling these three active neutrinos contribute as $N_{\text {eff }}=3.046$. Any departure of $N_{\text {eff }}$ from this last value would be due to non-standard neutrino features or to the contribution of other relativistic relics.

A detailed discussion of cosmological scenarios where $N_{\text {eff }}$ is not fixed to three can be found in the reviews [6,53], while the particular case of active-sterile neutrino mixing was recently analyzed in [54]. Since in the present work we focus on the standard case of three active neutrinos, here we only give a brief review of the most recent bounds on $N_{\text {eff }}$ from cosmological data.

The value of $N_{\text {eff }}$ is constrained at the BBN epoch from the comparison of theoretical predictions and experimental data on the primordial abundances of light elements, which also depend on the baryon-to-photon ratio $\eta_{\mathrm{b}}=n_{\mathrm{b}} / n_{\gamma}$ (or baryon density). The main effect of $N_{\text {eff }}$ is to fix the Hubble expansion rate through its contribution to the total energy density. This in turn changes the freezing temperature of the neutron-to-proton ratio, therefore producing a different abundance of ${ }^{4} \mathrm{He}$.

The BBN bounds on $N_{\text {eff }}$ have been recently reanalyzed taking in input the value of the baryon density derived from the WMAP first year data [55] $\eta_{\mathrm{CMB}}=6.14 \pm 0.25$. In Fig. 4 we show the results from [40], where the al- 


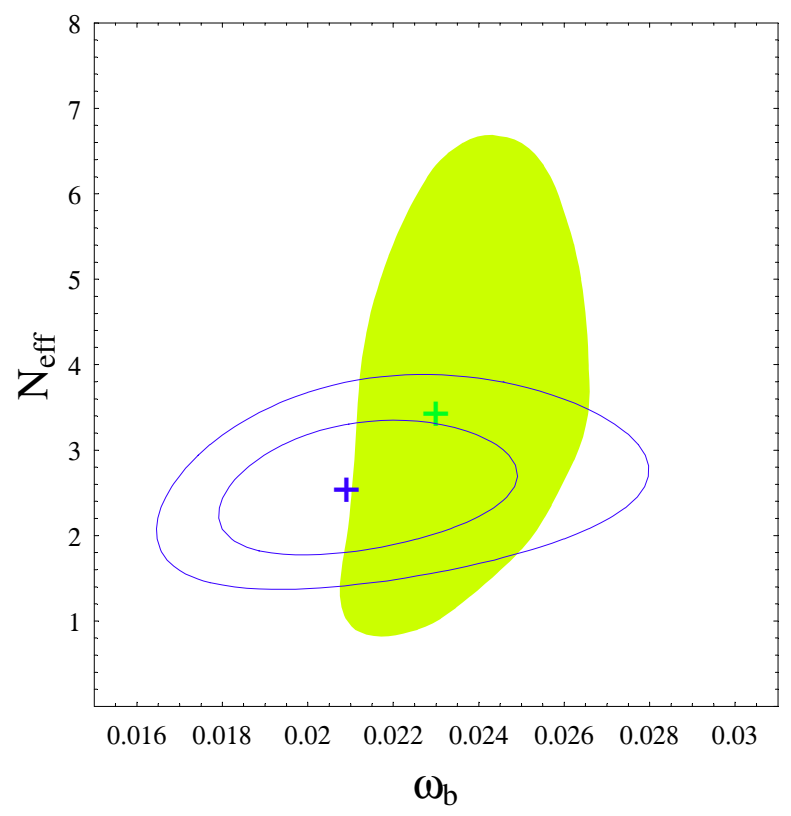

Fig. 4. Bounds on $N_{\text {eff }}$ from BBN including D and ${ }^{4} \mathrm{He}$ (contours at $68 \%$ and 95\% CL) and from a combined analysis of BBN (D only) and CMB data. Here $\omega_{\mathrm{b}}=\Omega_{\mathrm{b}} h^{2} \simeq 10^{10}\left(\eta_{\mathrm{b}} / 274\right)$. This Figure is taken from Ref. [40].

lowed range $N_{\text {eff }}=2.5_{-0.9}^{+1.1}(95 \%$ CL) was inferred from data on light element abundances (see also $[56,57,58]$ and $[59]$ for a recent review on BBN). This range is perfectly compatible with the standard prediction of 3.046. However, the reader should be cautious in the interpretation of the $\mathrm{BBN}$ allowed range for $N_{\text {eff }}$ such as that in Fig. 4. It is well-known that the main problem when deriving the primordial abundances from observations in astrophysical sources is the existence of systematics not accounted for, in particular for ${ }^{4} \mathrm{He}$ (a discussion of the observational situation can be found in Ref. [60]). For instance, the detailed BBN analysis in [61] gives the allowed range $1.90<N_{\text {eff }}<3.77$ for the ${ }^{4} \mathrm{He}$ "box" range in [60].

Independent bounds on the radiation content of the universe at a later epoch can be extracted from the analysis of the power spectrum of CMB anisotropies. We will describe later the neutrino effects on the CMB spectrum. Assuming a minimal set of cosmological parameters and a flat universe, Ref. [62] found the value $N_{\text {eff }}=3.5_{-2.1}^{+3.3}(95 \% \mathrm{CL})$ (see also $\left.[56,57,63]\right)$ from the combination of WMAP first year data with other CMB and LSS data. More recently, it has been shown that the addition of Supernovae and new CMB data leads to a reduction of the allowed range: $N_{\text {eff }}=4.2_{-1.7}^{+1.2}$ [64] (with the latest BOOMERANG data) and $N_{\text {eff }}=3.3_{-4.4}^{+0.9}[65]$ (with WMAP three year data). 


\subsection{Massive neutrinos as dark matter}

A priori, massive neutrinos are excellent candidates for contributing to the dark matter density, in particular because we are certain that they exist, in contrast with other candidate particles. Together with CMB photons, relic neutrinos can be found anywhere in the Universe with a number density of 339 neutrinos and antineutrinos per $\mathrm{cm}^{3}$. Depending on the value of the mass, this density is enhanced when neutrinos cluster into gravitational potential wells, although recent analyses show that the overdensity is limited to small factors (see e.g. [66]).

In addition, it is easy to have a neutrino contribution of order unity to the present value of the energy density of the Universe, just by considering $\mathrm{eV}$ neutrino masses. In such a case, we saw before that all neutrinos should approximately share the same mass $m_{0}$ and their energy density in units of the critical value of the energy density (see Eq. (21)) is ${ }^{1}$

$$
\Omega_{\nu}=\frac{\rho_{\nu}}{\rho_{\mathrm{c}}}=\frac{\sum_{i} m_{i}}{93.14 h^{2} \mathrm{eV}}
$$

where $h$ is the present value of the Hubble parameter in units of $100 \mathrm{~km} \mathrm{~s}^{-1}$ $\mathrm{Mpc}^{-1}$ and $\sum_{i} m_{i}=3 m_{0}$. Even if the three neutrinos are non-degenerate in mass, Eq. (12) can be safely applied. Indeed, we know from neutrino oscillation data that at least two of the neutrino states are non-relativistic today, since both $\left(\Delta m_{31}^{2}\right)^{1 / 2} \simeq 0.047 \mathrm{eV}$ and $\left(\Delta m_{21}^{2}\right)^{1 / 2} \simeq 0.009 \mathrm{eV}$ are larger than the temperature $T_{\nu} \simeq 1.96 \mathrm{~K} \simeq 1.7 \times 10^{-4} \mathrm{eV}$. If the third neutrino state is very light and still relativistic, its relative contribution to $\Omega_{\nu}$ is negligible and Eq. (12) remains an excellent approximation of the total density.

If we demand that neutrinos should not be heavy enough to overclose the Universe $\left(\Omega_{\nu}<1\right)$, we obtain an upper bound $m_{0} \lesssim 15 \mathrm{eV}$ for the absolute neutrino mass scale $m_{0}=\sum_{i} m_{i} / 3$ (fixing $h=0.7$ ). This argument was used many years ago by Gershtein and Zeldovich [67] (see also [68]). Since from present analysis of cosmological data we know that the approximate contribution of matter is $\Omega_{\mathrm{m}} \simeq 0.3$, the neutrino masses should obey the stronger bound $m_{0} \lesssim 5 \mathrm{eV}$.

Dark matter particles with a large velocity dispersion such as that of neutrinos are called hot dark matter (HDM). The role of neutrinos as HDM particles has been widely discussed since the 1970s, and the reader can find a historical review in Ref. [69]. It was realized in the mid-1980s (see e.g. [70,71,72]) that

$\overline{1}$ For high precision, the relation between $\left(m_{1}, m_{2}, m_{3}\right)$ and $\Omega_{\nu} h^{2}$ must be evaluated numerically using the distorted distributions described in [47]. 
HDM affects the evolution of cosmological perturbations in a particular way: it erases the density contrasts on wavelengths smaller than a mass-dependent free-streaming scale (we will discuss in more detail the effects of massive neutrinos on the evolution of cosmological perturbations in Secs. 4.5 and 4.6). In a universe dominated by HDM, this suppression is in contradiction with various observations. For instance, large objects such as superclusters of galaxies form first, while smaller structures like clusters and galaxies form via a fragmentation process. This top-down scenario is at odds with the fact that galaxies seem older than clusters.

Given the failure of HDM-dominated scenarios, the attention then turned to cold dark matter (CDM) candidates, i.e. particles which were non-relativistic at the epoch when the universe became matter-dominated, which provided a better agreement with observations. Still in the mid-1990s it appeared that a small mixture of HDM in a universe dominated by CDM fitted better the observational data on density fluctuations at small scales than a pure CDM model (see e.g. [73]). However, within the presently favoured $\Lambda$ CDM model dominated at late times by a cosmological constant (or some form of dark energy) there is no need for a significant contribution of HDM. Instead, one can use the available cosmological data to find how large the neutrino contribution can be. In Sec. 4, we will explain the effect of neutrino masses on cosmological observables. In Sec. 5, we will review the upper bounds on the sum of all neutrino masses which have been derived from current data.

\section{Massive neutrinos and cosmological perturbations}

Let us start with some generalities on the theory of cosmological perturbations. This field has been thoroughly investigated over the past thirty years, and many excellent reviews have been written on the subject (see for instance Refs. $[42,74,75,76,77])$. Many equations in this section are reminiscent of those in Ma \& Bertschinger [74] and Bertschinger [77] - however, with several sign differences, since we choose the metric signature to be $(+,-,-,-)$. This signature tends to be the most popular nowadays in cosmology.

In our Universe, the metric and the energy-momentum tensor are inhomogeneous. Their perturbations, given by

$$
\begin{aligned}
\delta g_{\mu \nu}(\mathbf{x}, t) & =g_{\mu \nu}(\mathbf{x}, t)-\bar{g}_{\mu \nu}(t) \\
\delta T_{\mu \nu}(\mathbf{x}, t) & =T_{\mu \nu}(\mathbf{x}, t)-\bar{T}_{\mu \nu}(t)
\end{aligned}
$$

are known to be small in the early Universe, typically $10^{5}$ times smaller than the background quantities, as shown by CMB anisotropies. As we shall see in 
this section, after photon decoupling, the matter perturbations grow by gravitational collapse and reach the non-linear regime, starting with the smallest scales. However, the linear perturbation theory is a good tool both for describing the early Universe at any scales, and the recent universe on the largest scales. The most reliable observations in cosmology are those involving mainly linear (or quasi-linear) perturbations. In particular, current cosmological neutrino mass bounds are based on such observations. Therefore, our goal in this section is to describe the evolution of linear cosmological perturbations. The great advantage of linear theory is, as usual, to obtain independent equations of evolution for each Fourier mode. Note that the Fourier decomposition must be performed with respect to the comoving coordinate system: so, the quantity $(2 \pi / k)$ is the comoving wavelength of a perturbation of wavevector $\mathbf{k}$, while the physical wavelength is given by

$$
\lambda=a(t) \frac{2 \pi}{k},
$$

where $a(t)$ is the scale factor of the Universe. For each mode $\mathbf{k}$, the perturbation amplitudes evolve under some equations of motion (which depend only on the modulus $k$, since the background is isotropic), and on top of this evolution, the physical wavelength is stretched according to the Universe expansion.

For concision, we will restrict ourselves to the main-stream standard cosmological model: the inflationary $\Lambda \mathrm{CDM}$ scenario, whose background evolution is reviewed in Sec. 4.2. In Sec. 5 we will briefly comment on some results based on more exotic scenarios. However, the theory of cosmological perturbations is so rich that in order to present a short and pedagogical summary, it is necessary to start from strong assumptions concerning the underlying cosmological background. Therefore, we will assume that at early times (e.g. at $\mathrm{BBN}$ ) the Universe contains a stochastic background of Gaussian, adiabatic ${ }^{2}$ and nearly scale-invariant primordial perturbations, as predicted by inflation, and as strongly suggested by CMB observations. Since the perturbations are stochastic and Gaussian, what we will call "amplitude" should often be understood as "variance": the linear equations of evolution give the evolution of each individual mode $\delta f_{\mathbf{k}}$ of a perturbation $\delta f$, and also, more interestingly, that of the root mean square $\left\langle|\delta f|^{2}\right\rangle_{k}^{1 / 2}$, obtained by averaging over all modes of fixed wavenumber $k$. It is this last quantity which is the target of most observations, and which carries information on the cosmological parameters.

The perturbations defined in Eqs. (13) contain many degrees of freedom: the

$\overline{2}$ If we relax the assumption of adiabaticity, i.e. if we allow for isocurvature modes in the early Universe, the neutrino background can play a very specific role and provides some non-trivial initial conditions for the evolution of cosmological perturbations. In this review, we will not cover this possibility and refer the interested reader to Refs. [78,79,80,81,82,83,84]. 
homogeneity and isotropy of the background implies that $\bar{g}_{\mu \nu}$ and $\bar{T}_{\mu \nu}$ are diagonal, but in general this is not true at the level of perturbations. However, we will see in Sec. 4.3 that some of these degrees of freedom are just artifacts of the relativistic perturbation theory set-up; moreover, only a fraction of the physical degrees of freedom contribute significantly to the CMB and LSS observables. So, we will see at the end of Sec. 4.3 that the problem can be reduced to the integration of a small number of linear equations of evolution.

In order to perform this integration, one must specify the properties of each fluid contributing to the energy density. Apart from the cosmological constant, the standard $\Lambda \mathrm{CDM}$ scenario includes contributions from photons, neutrinos, baryons and cold dark matter. In Sec. 4.4, we will review the behavior of the most relevant perturbations under the assumption that neutrinos are absent, in order to better identify the specific role of neutrinos in Sec. 4.5. In Sec. 4.6, we will summarize the effects of neutrino masses on cosmological observables, which are important for understanding the present bounds. Thus, the reader already familiar with cosmological perturbation theory in $\Lambda \mathrm{CDM}$ and $\Lambda \mathrm{MDM}$ $(\Lambda$ Mixed Dark Matter) models can go directly to the last subsection.

\subsection{Observables targets: definition of the power spectra}

Our goal is to compute observable quantities like

- the CMB temperature anisotropy power spectrum, defined as the angular two-point correlation function of CMB maps $\delta T / \bar{T}(\hat{n})$ ( $\hat{n}$ being a direction in the sky). This function is usually expanded in Legendre multipoles

$$
\left\langle\frac{\delta T}{\bar{T}}(\hat{n}) \frac{\delta T}{\bar{T}}\left(\hat{n}^{\prime}\right)\right\rangle=\sum_{l=0}^{\infty} \frac{(2 l+1)}{4 \pi} C_{l} P_{l}\left(\hat{n} \cdot \hat{n}^{\prime}\right),
$$

where $P_{l}(x)$ are the Legendre polynomials. So, for Gaussian fluctuations, all the information is encoded in the multipoles $C_{l}$ which probe correlations on angular scales $\theta=\pi / l$. Since for each neutrino family the mass is already known to be at most of the order of $1 \mathrm{eV}$ (see Sec. 5), the transition to the non-relativistic regime is expected to take place after the time of recombination between electrons and nucleons, i.e. after photon decoupling. The shape of the CMB spectrum is related mainly to the physical evolution before recombination. Therefore, the CMB will only be marginally affected by the neutrino mass (we will see later that there is only an indirect effect through the modified background evolution). For this reason, we will only mention in this review some essential aspects of the CMB temperature spectrum, without entering into details. For the effects on CMB of heavier neutrinos (with masses of a few eV), we refer the reader to Ref. [85]. 
- the CMB polarization anisotropy power spectra bring interesting complementary information to the temperature one, but for the same reasons as above, plus the fact that current polarization measurements are still rather imprecise, we will not discuss polarization in this section.

- the matter power spectrum, observed with various techniques described in the next section (directly or indirectly, today or in the near past), probes the current Large Scale Structure of the Universe. It is defined as the twopoint correlation function of non-relativistic matter fluctuations in Fourier space

$$
P(k, z)=\left\langle\left|\delta_{\mathrm{m}}(k, z)\right|^{2}\right\rangle
$$

where $\delta_{\mathrm{m}}=\delta \rho_{\mathrm{m}} / \bar{\rho}_{\mathrm{m}}$. In the case of several fluids (e.g. CDM, baryons and non-relativistic neutrinos), the total matter perturbation can be expanded as

$$
\delta_{\mathrm{m}}=\frac{\sum_{i} \bar{\rho}_{i} \delta_{i}}{\sum_{i} \bar{\rho}_{i}}
$$

Since the energy density is related to the mass density of non-relativistic matter through $E=m c^{2}, \delta_{\mathrm{m}}$ represents indifferently the energy or mass power spectrum. When the redshift $z$ is not explicitly written, we assume that $P(k)$ refers to the matter power spectrum evaluated today (at $z=0$ ). The shape of the matter power spectrum is the key observable for constraining small neutrino masses with cosmological methods. Therefore the main purpose of this section is to review the physics responsible for its shape, in a pedagogical but sufficiently detailed way, starting with linear structure formation in absence of neutrinos (Sec. 4.4), and then including the impact of free-streaming massive neutrinos (Secs. 4.5, 4.6).

- finally, one can build some cross-correlation power spectra (which measure the angular or spatial correlation between two different types of observables). These correlations are in principle very interesting to extract from observations, since they provide some additional information beyond that contained in the self-correlation power spectra $C_{l}$ or $P(k)$. For instance, the cross-correlation between CMB temperature and polarization maps is nonzero, since the two signals are affected by the same physical inhomogeneities close to the last-scattering surface. The CMB temperature maps are also correlated to some extent to the matter distribution at low redshift, due to the late integrated Sachs-Wolfe effect (that we will briefly introduce in Sec. 4.4.7). Finally, different measurements of the matter distribution in different overlapping redshift ranges or with different techniques are likely to be correlated with each other. The measurement of various cross-correlation power spectra is expected to be crucial in the future, but since nowadays it only plays a marginal role in neutrino mass determinations [86], we will not 
introduce the corresponding formalism in this review.

\subsection{Background evolution}

In most of this section, we will use the conformal time $\tau$ instead of the proper time $t$. The two are related through $d \tau=d t / a(t)$. A dot will denote a derivative with respect to $\tau$. For instance, the Hubble parameter reads $H=d(\ln a) / d t=\dot{a} / a^{2}$. In absence of spatial curvature, the background Friedmann metric reduces to

$$
d s^{2}=a(\tau)^{2}\left[d \tau^{2}-\delta_{i j} d x^{i} d x^{j}\right]
$$

and the Friedmann equation reads

$$
H^{2}=\frac{8 \pi G}{3}\left(\rho_{\gamma}+\rho_{\mathrm{cdm}}+\rho_{\mathrm{b}}+\rho_{\nu}+\rho_{\Lambda}\right)
$$

where the homogeneous density of photons $\rho_{\gamma}$ scales like $a^{-4}$, that of nonrelativistic matter ( $\rho_{\mathrm{cdm}}$ for CDM and $\rho_{\mathrm{b}}$ for baryons) like $a^{-3}$, that of neutrinos $\rho_{\nu}$ interpolates between the two behaviors at the time of the nonrelativistic transition, and finally the cosmological constant density $\rho_{\Lambda}$ is of course time-independent. At any time, the critical density $\rho_{c}$ is defined as $\rho_{\mathrm{c}}=3 H^{2} / 8 \pi G$, and the current value $H_{0}$ (or reduced value $h$ ) of the Hubble parameter gives the critical density today

$$
\rho_{\mathrm{c}}^{0}=1.8788 \times 10^{-29} h^{2} \mathrm{~g} \mathrm{~cm}^{-3} .
$$

The normalization of the scale factor $a$ is arbitrary. If we first neglect neutrino masses, the photon and neutrino densities can be combined into a total radiation density $\rho_{\mathrm{r}}=\rho_{\gamma}+\rho_{\nu}$ scaling like $a^{-4}$, while the CDM and baryons combine into a matter density $\rho_{\mathrm{m}}=\rho_{\mathrm{cdm}}+\rho_{\mathrm{b}}$ scaling like $a^{-3}$. Then, we can choose the normalization of $a$ such that $\rho_{\mathrm{m}}=3 /\left(8 \pi G a^{3}\right)$ in order to obtain a particularly simple Friedmann equation valid during radiation domination (RD) and matter domination (MD)

$$
(\dot{a})^{2}=a+a_{\mathrm{eq}},
$$

where $a_{\mathrm{eq}}$ is the scale factor at equality, when $\rho_{\mathrm{m}}=\rho_{\mathrm{r}}$. There is a simple solution,

$$
a=\frac{\tau^{2}}{4}+\sqrt{a_{\mathrm{eq}}} \tau=\frac{\tau}{4}\left(\tau+2(\sqrt{2}+1) \tau_{\mathrm{eq}}\right)
$$



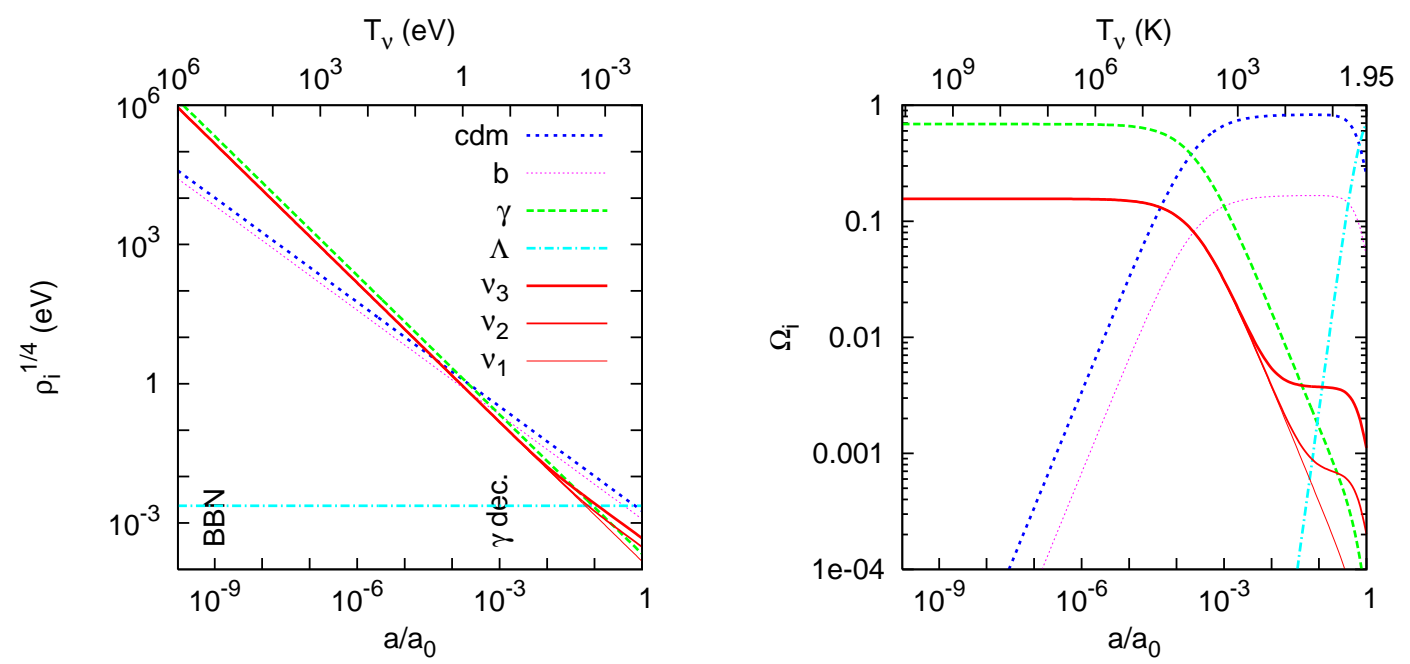

Fig. 5. Evolution of the background densities from the time when $T_{\nu}=1 \mathrm{MeV}$ (soon after neutrino decoupling) until now, for each component of a flat $\Lambda$ MDM model with $h=0.7$ and current density fractions $\Omega_{\Lambda}=0.70, \Omega_{\mathrm{b}}=0.05, \Omega_{\nu}=0.0013$ and $\Omega_{\mathrm{cdm}}=1-\Omega_{\Lambda}-\Omega_{\mathrm{b}}-\Omega_{\nu}$. The three neutrino masses are distributed according to the Normal Hierarchy scheme (see Sec. 2) with $m_{1}=0, m_{2}=0.009 \mathrm{eV}$ and $m_{3}=0.05 \mathrm{eV}$. On the left plot we show the densities to the power $1 / 4$ (in $\mathrm{eV}$ units) as a function of the scale factor. On the right plot, we display the evolution of the density fractions (i.e., the densities in units of the critical density). We also show on the top axis the neutrino temperature (on the left in $\mathrm{eV}$, and on the right in Kelvin units). The density of the neutrino mass states $\nu_{2}$ and $\nu_{3}$ is clearly enhanced once they become non-relativistic. On the left plot, we also display the characteristic times for the end of BBN and for photon decoupling or recombination.

where $\tau_{\text {eq }}=2(\sqrt{2}-1) \sqrt{a_{\text {eq }}}$ is the value of the conformal time at equality. At low redshift (typically $z<0.5$ ), the cosmological constant density takes over, causing a departure from the above solution, with an acceleration of the scale factor. Finally, if we include the effect of small neutrino masses, the solution is also slightly modified, since the non-relativistic transition of each neutrino species amounts in converting a fraction of radiation into matter. This can be seen in Fig. 5, where we plot the evolution of background densities for a $\Lambda \mathrm{MDM}$ model in which the three neutrino masses follow the Normal Hierarchy scheme (see Sec. 2) with $m_{1}=0, m_{2}=0.009 \mathrm{eV}$ and $m_{3}=0.05 \mathrm{eV}$.

\subsection{Gauge transformations and Einstein equations}

In the real Universe all physical quantities (densities, curvature...) are functions of time and space. Thanks to the covariance of general relativity, they can be described in principle in any coordinate system, without changing the physical predictions. The problem is that in order to obtain simple equa- 
tions of evolution, we wish to use a linear perturbation theory, in which the true physical quantities are artificially decomposed into a homogeneous background and some small perturbations. This is artificial because the homogeneous quantities are defined as spatial averages over hypersurfaces of simultaneity: $\bar{f}(t)=\langle f(t, \mathbf{x})\rangle_{\mathbf{x}}$. Any change of coordinate system which

(1) mixes time and space (therefore, redefining hypersurfaces of simultaneity, and changing the way to perform spatial averages), and

(2) remains small everywhere, so that the differences between true quantities and spatial averages are still small perturbations,

gives a new set of perturbations (new equations of evolution, new initial conditions), although the physical quantities (i.e., the total ones) are the same. This ambiguity is called the gauge freedom in the context of relativistic perturbation theory.

Of course, using a linear perturbation theory is only possible when there exists at least one system of coordinates in which the Universe looks approximately homogeneous. We know that this is the case at least until the time of photon decoupling: in some reference frames, the CMB anisotropies do appear as small perturbations. It is a necessary condition for using linear theory to be in such a frame; however, this condition is vague and leaves a lot of gauge freedom, i.e. many possible ways to slice the spacetime into hypersurfaces of simultaneity.

We can also notice that the definition of hypersurfaces of simultaneity is not ambiguous at small distances, as long as different observers can exchange light signals in order to synchronize their clocks. Intuitively, we see that the gauge freedom is an infrared problem, since on very large distances (larger than the Hubble distance) the word "simultaneous" does not have a clear meaning. The fact that the gauge ambiguity is only present on large scales emerges naturally from the mathematical framework describing gauge transformations.

Formally, a gauge transformation is described by a quadrivector field $\epsilon^{\mu}(\mathbf{x}, t)$ (see e.g. Ref. [74]). When the latter is infinitesimal, the Lorentz scalars, vectors and tensors describing the perturbations are shifted by the Lie derivative along $\epsilon$,

$$
\delta A_{\mu \nu \ldots}(\mathbf{x}, t) \rightarrow \delta A_{\mu \nu \ldots}(\mathbf{x}, t)+\mathcal{L}_{\epsilon}\left[\delta A_{\mu \nu \ldots}(\mathbf{x}, t)\right]
$$

Since there are four degrees of freedom (d.o.f.) in this transformation - the four components of $\epsilon^{\mu}$ - we see that among the ten d.o.f. of the perturbed Einstein equation $\delta G_{\mu \nu}=8 \pi G \delta T_{\mu \nu}$, four represent gauge modes, and six represent physical degrees of freedom. 
In addition, it can be shown that this equation contains three decoupled sectors. In other words, when the metric and the energy-momentum tensor are parametrized in an adequate way, the ten equations can be decomposed into three systems independent of each other:

(1) four equations relate four scalars in the perturbed metric $\delta g_{\mu \nu}$ to four scalars in $\delta T_{\mu \nu}$,

(2) four equations relate two transverse 3-vectors in the perturbed metric (4 d.o.f. in total) to two transverse 3-vectors in $\delta T_{\mu \nu}$,

(3) two equations relate one transverse traceless $3 \times 3$-tensor in the perturbed metric (2 d.o.f. in total) to a similar tensor in $\delta T_{\mu \nu}$.

Moreover, this decomposition is left invariant by gauge transformations. These three types of variables are called scalar, vector and tensor modes; physically, they describe respectively the generalization of Newtonian gravity, gravitomagnetism, and gravitational waves. It can be shown that two scalar d.o.f. and two vector d.o.f. are gauge modes, so each of the three sectors contains only two physical d.o.f. In principle, all modes can contribute to the CMB anisotropy maps, but CMB observations show that the vector and tensor contributions are negligible (at least for temperature anisotropies). As for the LSS of the Universe, it is related to the mass/energy density distribution of non-relativistic matter, i.e., again to the scalar sector. Therefore, in this report, we will focus only on scalar perturbations, not because the cosmological backgrounds of vector/tensor modes are insensitive to neutrino properties, but because they are not likely to be observed with great precision in a near future.

It is possible to build some gauge-invariant combinations, and to reduce the Einstein equation into a set of gauge-invariant equations. This is not the most economic way to proceed: one can simply choose an arbitrary gauge-fixing condition, i.e., a prescription that will limit the number of effective degrees of freedom to that of physical modes only, and make all calculations into that gauge. When the same problem is studied in two different gauges, the solutions can look very different on large wavelength; for instance, the total energy density perturbation $\delta \rho(t, k)$ for a given time and wavenumber can appear as growing in one gauge, and constant in another gauge, although the two solutions describe the same Universe. However, physical observables - like the matter density perturbations probed by galaxy surveys or temperature/polarization anisotropies probed by CMB experiments - are always limited to small scales, at most of the order of the Hubble length. On those scales, the predictions arising from different gauge choices always coincide with each other.

Throughout our discussion, we choose to work in the longitudinal gauge, which is probably the most popular for studying cosmological perturbations. In this 
gauge, one requires that the non-diagonal metric perturbations vanish. This eliminates two scalar degrees of freedom, and the remaining ones are defined in such a way that Eq. (19) reads

$$
d s^{2}=g_{\mu \nu} d x^{\mu} d x^{\nu}=a^{2}(\tau)\left[(1+2 \phi) d \tau^{2}-(1-2 \psi) \delta_{i j} d x^{i} d x^{j}\right]
$$

For the energy-momentum tensor, the four scalar degrees of freedom can be identified as

$$
\begin{aligned}
\delta T_{0}^{0} & =\delta \rho \\
\delta T_{i}^{0} & =(\bar{\rho}+\bar{p}) v_{i}^{\|}, \\
\delta T_{j}^{i} & =-\delta p \delta_{j}^{i}+\Sigma_{j}^{i \|},
\end{aligned}
$$

where $\delta \rho$ is the energy density perturbation, $\delta p$ the pressure perturbation, $v_{i}^{\|}=$ $\partial_{i} \tilde{v}$ the longitudinal component of the velocity field, and $\Sigma_{j}^{i \|}=\left(\partial_{i} \partial_{j}-\frac{1}{3} \delta_{i j} \nabla^{2}\right) \tilde{\sigma}$ the traceless and longitudinal-divergence component of the $3 \times 3$ tensor $\delta T_{j}^{i}$. For these last two degrees of freedom, we could write the equations in terms of the two potentials $\tilde{v}$ and $\tilde{\sigma}$. However, it is more conventional to deal with the two quantities $(\theta, \sigma)$ which represent respectively the velocity divergence and the shear stress (or anisotropic stress)

$$
\begin{aligned}
\theta & \equiv \sum_{i} \partial_{i} v_{i}=\nabla^{2} \tilde{v} \\
(\bar{\rho}+\bar{p}) \nabla^{2} \sigma & \equiv-\sum_{i, j}\left(\partial_{i} \partial_{j}-\frac{1}{3} \nabla^{2} \delta_{i j}\right) \Sigma_{j}^{i \|}=-\frac{2}{3} \nabla^{4} \tilde{\sigma}
\end{aligned}
$$

Let us recall that the four scalar components of the energy-momentum tensor are not gauge-invariant: we define the above quantities in the longitudinal gauge, but in another gauge, for instance, $\delta T_{0}^{0}$ could behave differently than the above $\delta \rho$, especially on super-Hubble scales.

We can now write the perturbed Einstein equation for the scalar sector in the longitudinal gauge,

$$
\begin{aligned}
\delta G_{0}^{0}= & 2 a^{-2}\left\{-3\left(\frac{\dot{a}}{a}\right)^{2} \phi-3 \frac{\dot{a}}{a} \dot{\psi}+\nabla^{2} \psi\right\}=8 \pi G \delta \rho \\
\delta G_{i}^{0}= & 2 a^{-2} \partial_{i}\left\{\frac{\dot{a}}{a} \phi+\dot{\psi}\right\}=8 \pi G(\bar{\rho}+\bar{p}) v_{i}, \\
\delta G_{j}^{i}= & -2 a^{-2}\left\{\left[\left(2 \frac{\ddot{a}}{a}-\left(\frac{\dot{a}}{a}\right)^{2}\right) \phi+\frac{\dot{a}}{a}(\dot{\phi}+2 \dot{\psi})+\ddot{\psi}+\frac{1}{3} \nabla^{2}(\phi-\psi)\right] \delta_{j}^{i}\right. \\
& \left.-\frac{1}{2}\left(\partial^{i} \partial_{j}-\frac{1}{3} \nabla^{2} \delta_{j}^{i}\right)(\phi-\psi)\right\}
\end{aligned}
$$




$$
=8 \pi G\left(-\delta p \delta_{j}^{i}+\Sigma_{j}^{i}\right)
$$

Using the variables $\delta \equiv \delta \rho / \bar{\rho}, \delta p, \theta, \sigma$, and switching to comoving Fourier space, we obtain

$$
\begin{aligned}
-3\left(\frac{\dot{a}}{a}\right)^{2} \phi-3 \frac{\dot{a}}{a} \dot{\psi}-k^{2} \psi & =4 \pi G a^{2} \bar{\rho} \delta \\
-k^{2}\left(\frac{\dot{a}}{a} \phi+\dot{\psi}\right) & =4 \pi G a^{2}(\bar{\rho}+\bar{p}) \theta \\
\left(2 \frac{\ddot{a}}{a}-\left(\frac{\dot{a}}{a}\right)^{2}\right) \phi+\frac{\dot{a}}{a}(\dot{\phi}+2 \dot{\psi})+\ddot{\psi}-\frac{k^{2}}{3}(\phi-\psi) & =4 \pi G a^{2} \delta p \\
k^{2}(\phi-\psi) & =12 \pi G a^{2}(\bar{\rho}+\bar{p}) \sigma
\end{aligned}
$$

Through the Bianchi identities, the Einstein equation implies the conservation of the total energy-momentum tensor. Actually, the energy-momentum tensor of each uncoupled fluid is conserved, and obeys the continuity equation

$$
\dot{\delta}=(1+w)(\theta+3 \dot{\psi})
$$

and the Euler equation

$$
\dot{\theta}=\frac{\dot{a}}{a}(3 w-1) \theta-\frac{\dot{w}}{1+w} \theta-k^{2} \phi-k^{2} \sigma-\frac{w}{1+w} k^{2} \delta
$$

where we assumed an equation of state $p(x, t)=w \rho(x, t)$ for the fluid, so that $w=\bar{p} / \bar{\rho}=\delta p / \delta \rho$.

\subsection{Linear perturbation theory in a neutrinoless Universe (pure $\Lambda$ CDM)}

\subsubsection{Perfect fluids}

Intuitively, in perfect fluids, microscopic interactions enforce a "collective behavior" of the particles. More precisely, they guarantee that the stress tensor $T_{i j}$ is isotropic and diagonal, and that the energy-momentum tensor can be simply described in terms of functions of time and space: the density $\rho$, the pressure $p$ and the bulk velocity $U^{\mu}$,

$$
T^{\mu \nu}=-p g^{\mu \nu}+(\rho+p) U^{\mu} U^{\nu}
$$

Here, $U^{\mu}=d x^{\mu} / \sqrt{d s^{2}}$ is the 4-velocity. As long as the bulk 3-velocity $v^{i}=$ $d x^{i} / d \tau$ remains first-order in perturbations, we can approximate the 4 -velocity 
by $U^{\mu}=d x^{\mu} /[a(1+\phi) d \tau]$ and obtain:

$$
U^{\mu}=\left(a^{-1}[1-\phi], a^{-1} v^{i}\right), \quad T_{0}^{0}=\rho, \quad T_{0}^{i}=v^{i}, \quad T_{i}^{i}=-p
$$

where $T_{i}^{i}$ is not summed over the repeated index. In a pure $\Lambda$ CDM model, the Universe contains photons, baryons and cold dark matter. Strictly speaking, the perfect fluid approximation does not apply to this situation, since on the one hand photons become collisionless after decoupling, and on the other hand cold dark matter is collisionless at any time (so it cannot even be called a fluid). However, we will see in the next paragraphs that we can use Eqs. $(41,42)$ in an effective way.

First, after its own decoupling time, the cold dark matter component is always non-relativistic and collisionless. As long as the bulk motion of CDM particles can be described by a single-valued flow, the CDM energy-momentum tensor is formally identical to that of a perfect fluid with density $\rho_{\mathrm{m}}$ and zero pressure.

Second, let us discuss the case of photons and baryons. If our purpose was to review the CMB physics, it would be crucial to introduce separately the exact form of the photon and baryon energy-momentum tensors. However, in this report, we only wish to provide a simplified description of large scale structure formation. For this purpose, we can view the baryon and photon components before recombination as forming a single tightly-coupled fluid, obeying to Eq. (41) with the equation of state of ordinary radiation: $p_{\mathrm{r}}=\frac{1}{3} \rho_{\mathrm{r}}$ (this assumes that the baryon density is negligible with respect to the photon density, which is only a crude approximation around the time of equality and recombination). The local value of $\rho_{\mathrm{r}}$ is related to the local value of the photon blackbody temperature. The bulk velocity of this fluid remains small enough to use Eqs. (42). After recombination, the photons are irrelevant for structure formation, and we will simply discard this component; the baryons behave like a non-relativistic collisionless medium, but for simplicity we will neglect their density with respect to that of cold dark matter.

Finally, since we are only interested in scalar perturbations, we can reduce the bulk velocities of radiation and CDM into irrotational vector fields, and define two scalars $\theta_{\mathrm{r}}$ and $\theta_{\mathrm{m}}$ as in Eq. (29).

In summary, we can solve the Einstein equations with the following perturbed energy-momentum tensor

$$
\begin{aligned}
\delta T_{0}^{0} & =\delta \rho_{\mathrm{r}}+\delta \rho_{\mathrm{m}} \\
\partial^{i}\left(\delta T_{i}^{0}\right) & =\left(\bar{\rho}_{\mathrm{r}}+\bar{p}_{\mathrm{r}}\right) \theta_{\mathrm{r}}+\bar{\rho}_{\mathrm{m}} \theta_{\mathrm{m}}=\frac{4}{3} \bar{\rho}_{\mathrm{r}} \theta_{\mathrm{r}}+\bar{\rho}_{\mathrm{m}} \theta_{\mathrm{m}},
\end{aligned}
$$




$$
\delta T_{i}^{i}=-\delta p_{\mathrm{r}}=-\frac{1}{3} \delta \rho_{\mathrm{r}}
$$

\subsubsection{Jeans length}

In a spatially flat Friedmann Universe, a physical process switched on at a time $t_{i}$ and propagating at a velocity $v$ along radial geodesics $(v d t=a(t) d x)$ can only affect wavelengths smaller than a causal horizon, defined as

$$
d\left(t_{i}, t\right)=a(t) \int_{t_{i}}^{t} d x=a(t) \int_{t_{i}}^{t} \frac{v d t^{\prime}}{a\left(t^{\prime}\right)}
$$

This horizon is simply the maximal physical distance on which the signal can propagate between $t_{i}$ and $t$ (sometimes, is it defined with a factor two bigger than above). The so-called particle horizon $d_{H}$ obeys this definition in the particular case of a signal traveling at the speed of light, $v=1$ (we adopted units in which $c=1$ ). If both $t_{i}$ and $t$ are chosen during the matter or radiation dominated stage, and if $t \gg t_{i}$, it is straightforward to show that the particle horizon can be approximated by the Hubble length $R_{H}(t)=1 / H(t)$, up to a numerical factor of order one. Indeed, for a power-law expansion $a(t) \propto t^{n}$ with $n<1$, one has

$$
R_{H}(t)=\frac{t}{n}, \quad d_{H}\left(t \gg t_{i}\right) \simeq \frac{t}{1-n} .
$$

Similarly, the characteristic velocity $c_{s}$ under which acoustic perturbations propagate before photon decoupling defines a characteristic length $d_{s}\left(t_{i}, t\right)$, called the sound horizon. For a constant sound speed, and under the conditions described above for $t_{i}$ and $t$, the sound horizon is obviously given (up to a numerical factor of order one) by the ratio $c_{s} / H(t)$, which is called the Jeans length. The exact definition of the Jeans wavenumber and Jeans length is usually

$$
k_{J}(t)=\left(\frac{4 \pi G \bar{\rho}(t) a^{2}(t)}{c_{s}^{2}(t)}\right)^{1 / 2}, \quad \lambda_{J}(t)=2 \pi \frac{a(t)}{k_{J}(t)}=2 \pi \sqrt{\frac{2}{3}} \frac{c_{s}(t)}{H(t)},
$$

where the numerical factors - which are unimportant for the purpose of understanding the physics- are simply dictated by the particular form of the Newtonian equation of evolution of the density contrast of a single uncoupled perfect fluid with constant sound speed,

$$
\ddot{\delta}+\frac{\dot{a}}{a} \dot{\delta}+\left(k^{2}-k_{J}^{2}\right) c_{s}^{2} \delta=0
$$


which derives from the combination of the continuity, Euler and Poisson equations.

Our physical expectation is that for a fluid of sound speed $c_{s}$, modes with $k>k_{J}$ will oscillate with a pulsation $\omega=k c_{s}$, due to the competition between the gas pressure and the gravitational compression. These modes are said to be Jeans stable. On the other hand, for modes $k<k_{J}$, pressure cannot causally resist to gravitational compression, and density perturbations should in principle grow monotonically. This Jeans instability explains some basic phenomena in the inhomogeneous Universe: before recombination, the photonbaryon fluid has a sound speed of order $c_{s} \sim c / \sqrt{3}$ and oscillates on scales smaller than $\lambda_{J}$; after recombination, $c_{s}$ and $\lambda_{J}$ become vanishingly small, $k_{J}$ grows to infinity and structures form.

\subsubsection{Radiation domination}

Deep inside the radiation era, we have seen that the radiation component can be approximated by a perfect fluid of sound speed $c_{s}$, due to the tight coupling between baryon and photons. This self-gravitating fluid oscillates inside the Jeans length (or sound horizon). The cold dark matter evolves as a test fluid in this background, until the time (close to equality) at which its gravitational back-reaction becomes important. However, for simplicity, we will work at first order in the expansion parameter $\bar{\rho}_{\mathrm{m}} / \bar{\rho}_{\mathrm{r}}$.

In this limit, it is possible to derive an analytic expression for the evolution of $\delta_{\mathrm{r}}$ and $\delta_{\mathrm{m}}$. The absence of anisotropic stress implies that the two metric fluctuations are equal, $\phi=\psi$. The full continuity, Euler and Einstein equations for the radiation and matter fluid read

$$
\begin{aligned}
& \dot{\delta}_{\mathrm{m}}=\theta_{\mathrm{m}}+3 \dot{\phi}, \dot{\delta}_{\mathrm{r}}=\frac{4}{3} \theta_{\mathrm{r}}+4 \dot{\phi} \\
& \dot{\theta}_{\mathrm{m}}=-\frac{\dot{a}}{a} \theta_{\mathrm{m}}-k^{2} \phi, \dot{\theta}_{\mathrm{r}}=-\frac{k^{2}}{4} \delta_{\mathrm{r}}-k^{2} \phi \\
&-3 \frac{\dot{a}}{a} \dot{\phi}-\left(3\left(\frac{\dot{a}}{a}\right)^{2}+k^{2}\right) \phi=4 \pi G a^{2}\left(\bar{\rho}_{\mathrm{m}} \delta_{\mathrm{m}}+\bar{\rho}_{\mathrm{r}} \delta_{\mathrm{r}}\right) \\
&-k^{2}\left(\dot{\phi}+\frac{\dot{a}}{a} \phi\right)=4 \pi G a^{2}\left(\bar{\rho}_{\mathrm{m}} \theta_{\mathrm{m}}+\frac{4}{3} \bar{\rho}_{\mathrm{r}} \theta_{\mathrm{r}}\right) \\
& \ddot{\phi}+3 \frac{\dot{a}}{a} \dot{\phi}+\left(2 \frac{\ddot{a}}{a}-\left(\frac{\dot{a}}{a}\right)^{2}\right) \phi=4 \pi G a^{2}\left(\frac{1}{3} \bar{\rho}_{\mathrm{r}} \delta_{\mathrm{r}}\right)
\end{aligned}
$$

and only five out of these seven equations are independent. In addition, one out of the five independent equations is just a constraint equation, so the system admits four independent solutions. In order to find them, let us first notice that since we have two fluids, we can have differences in the number 
density contrasts, usually parametrized by the entropy perturbation

$$
\eta=\frac{3}{4} \delta_{\mathrm{r}}-\delta_{\mathrm{m}}
$$

The pressure perturbation $\delta p=\delta p_{\mathrm{r}}$ can be uniquely decomposed in the basis of the total density perturbation $\delta \rho=\delta \rho_{\mathrm{r}}+\delta \rho_{\mathrm{m}}$ and entropy perturbation $\eta$,

$$
\delta p=\left[3\left(1+\frac{3 \bar{\rho}_{\mathrm{m}}}{4 \bar{\rho}_{\mathrm{r}}}\right)\right]^{-1}\left(\delta \rho+\bar{\rho}_{\mathrm{m}} \eta\right) .
$$

This defines the effective sound speed in the two-fluid as

$$
c_{\text {eff }}^{2}=\left[3\left(1+\frac{3 \bar{\rho}_{\mathrm{m}}}{4 \bar{\rho}_{\mathrm{r}}}\right)\right]^{-1}=\frac{\dot{\bar{p}}}{\dot{\bar{\rho}}} .
$$

We can reduce the system (50)-(54) to a pair of second-order coupled equations, obtained from the combinations $\left[(54)-c_{\mathrm{eff}}^{2}(52)\right]$ and $\left[k^{2}(52)-3 \dot{a} / a(53)\right]$, as follows

$$
\begin{aligned}
& \ddot{\phi}+3 \frac{\dot{a}}{a}\left(1+c_{\mathrm{eff}}^{2}\right) \dot{\phi}+\frac{1}{a}\left[3\left(1+\frac{\bar{\rho}_{\mathrm{r}}}{\bar{\rho}_{\mathrm{m}}}\right) c_{\mathrm{eff}}^{2}-\frac{\bar{\rho}_{\mathrm{r}}}{\bar{\rho}_{\mathrm{m}}}\right] \phi+k^{2} c_{\mathrm{eff}}^{2} \phi=\frac{3}{2 a} c_{\mathrm{eff}}^{2} \eta, \\
& \frac{1}{3 c_{\mathrm{eff}}^{2}} \ddot{\eta}+\frac{\dot{a}}{a} \dot{\eta}+\frac{k^{2}}{4} \frac{\bar{\rho}_{\mathrm{m}}}{\bar{\rho}_{\mathrm{r}}} \eta=\frac{k^{4}}{6} a \frac{\bar{\rho}_{\mathrm{m}}}{\bar{\rho}_{\mathrm{r}}} \phi .
\end{aligned}
$$

Once the four independent solutions have been found, the density contrasts can be obtained e.g. from combining Eqs. (54) and (58)

$$
\begin{aligned}
\delta_{\mathrm{r}} & =\frac{3 c_{\mathrm{eff}}^{2}}{4 \pi G a^{2} \bar{\rho}_{\mathrm{r}}}\left(\frac{3}{2 a} \eta-3 \frac{\dot{a}}{a} \dot{\phi}-\left(3\left(\frac{\dot{a}}{a}\right)^{2}+k^{2}\right) \phi\right), \\
\delta_{\mathrm{m}} & =\frac{9 c_{\mathrm{eff}}^{2}}{16 \pi G a^{2} \bar{\rho}_{\mathrm{r}}}\left(\frac{3}{2 a} \eta-3 \frac{\dot{a}}{a} \dot{\phi}-\left(3\left(\frac{\dot{a}}{a}\right)^{2}+k^{2}\right) \phi\right)-\eta,
\end{aligned}
$$

and the velocity gradients follow from Eqs. (51). The evolution of the scale factor can be taken from equation (23). Deep inside the radiation era, we can write $a, c_{\text {eff }}^{2}$ and the two equations $\left[\tau^{2} \times(58)\right]$ and (59) at first order in $\bar{\rho}_{\mathrm{m}} / \bar{\rho}_{\mathrm{r}}=\tau / \tau_{\text {eq }}$,

$$
\tau^{2} \ddot{\phi}+4 \tau \dot{\phi}+\left(\tau^{2} \omega^{2}\right) \phi=0, \quad \ddot{\eta}+\frac{1}{\tau} \dot{\eta}=\frac{3 \omega^{4} \tau^{2}}{2} \phi
$$

where $\omega=k c_{\text {eff }}=k / \sqrt{3}$. In this limit, the first equation decouples: this just confirms that the radiation fluid is self-gravitating, and the CDM behaves as 
a test fluid. Two independent solutions of the system, both satisfying $\eta(\tau \rightarrow$ $0)=0$, read

$$
\begin{aligned}
& \phi=(\omega \tau)^{-2}\left\{C(k)\left(\frac{\sin \omega \tau}{\omega \tau}-\cos \omega \tau\right)+D(k)\left(-\sin \omega \tau-\frac{\cos \omega \tau}{\omega \tau}\right)\right\}, \\
& \eta=-3\left\{C(k)\left(\int_{0}^{\omega \tau} \frac{\cos x-1}{x} d x-\frac{1}{2}(\cos \omega \tau-1)\right)\right. \\
&\left.+D(k)\left(\int_{0}^{\omega \tau} \frac{\sin x}{x} d x+\frac{1}{2} \sin \omega \tau\right)\right\} .
\end{aligned}
$$

The solution proportional to $C(k)$ represents the growing adiabatic (or isentropic) mode, the other one is the decaying adiabatic mode. There are two other obvious solutions $(\phi, \eta)=(0, A(k))$ and $(\phi, \eta)=(0, B(k) \ln \tau)$ which stand respectively for the isocurvature growing and decaying mode. For the growing adiabatic mode, we get from Eqs. (60) and (61) that the radiation and matter density contrasts evolve like

$$
\begin{gathered}
\delta_{\mathrm{r}}=-2 C(k)\left\{\frac{2 \sin \omega \tau-\omega \tau \cos \omega \tau}{\omega \tau}-2 \frac{\sin \omega \tau-\omega \tau \cos \omega \tau}{(\omega \tau)^{3}}\right\}, \\
\delta_{\mathrm{m}}=3 C(k)\left\{-\frac{\sin \omega \tau}{\omega \tau}+\frac{\sin \omega \tau-\omega \tau \cos \omega \tau}{(\omega \tau)^{3}}+\frac{1}{2}+\int_{0}^{\omega \tau} \frac{\cos x-1}{x} d x\right\} .
\end{gathered}
$$

The integral is given by

$$
\int_{0}^{\omega \tau} \frac{\cos x-1}{x} d x=\operatorname{Ci}(\omega \tau)-\mathcal{C}-\ln (\omega \tau)
$$

where $\mathrm{Ci}(x)$ is the cosine integral and $\mathcal{C} \simeq 0.5772$ is the Euler constant. For $x>1$, the function $\mathrm{Ci}(x)$ describes damped oscillations around zero. Let us summarize the physics described by these solutions. During the radiation era and far outside the sound horizon, all quantities appear as frozen in the longitudinal gauge, with

$$
\delta_{\mathrm{m}}=\frac{3}{4} \delta_{\mathrm{r}}=-\frac{3}{2} \phi=-\frac{1}{2} C(k) . \quad[\mathrm{RD}, \quad \omega \tau=k \tau / \sqrt{3} \ll 1]
$$

Then, inside the sound horizon, the evolution of the radiation and matter perturbations are radically different from each other. The self-gravitating radiation component oscillates on scales smaller than its Jeans length, and Eq. (64) represents a first-order approximation to the acoustic oscillations observed in the CMB anisotropy spectrum. Instead, the cold dark matter has 
a considerably smaller Jeans length and does not fluctuate on its own. The gravitational driving force only induces damped oscillations on $\delta_{\mathrm{m}}$, on top of a net gravitational clustering effect. This clustering is not very efficient, because the gravitational potential fluctuations are damped by the acoustic oscillations and do not feel significant back-reaction from the CDM component. In average, the growth of the CDM perturbations is proportional to the logarithm of conformal time

$$
\delta_{\mathrm{m}} \simeq 3 C(k)[1 / 2+\mathcal{C}+\ln (\omega \tau)] . \quad[\mathrm{RD}, \quad \omega \tau=k \tau / \sqrt{3} \gg 1]
$$

\subsubsection{Matter domination}

Deep into the matter domination era, when $a \propto \tau^{2}$, the effective sound speed is vanishingly small and Eq. (58) simply reduces to

$$
\ddot{\phi}+3 \frac{\dot{a}}{a} \dot{\phi}=0
$$

with solutions

$$
\phi=\tilde{C}(k)+\tau^{-5} \tilde{D}(k)
$$

The matter density contrast then follows from Eq. (52), which simplifies into

$$
-\left(\frac{12}{\tau^{2}}+k^{2}\right) \phi-\frac{6}{\tau} \dot{\phi}=\frac{3}{\tau^{2}} \delta_{\mathrm{m}}
$$

and we finally obtain

$$
\delta_{\mathrm{m}}=-\left(4+\frac{k^{2} \tau^{2}}{3}\right) \tilde{C}(k)+\left(\frac{k^{2} \tau^{2}}{3}-6\right) \tau^{-5} \tilde{D}(k)
$$

We can identify the solution proportional to $\tilde{C}(k)$ (resp. $\tilde{D}(k))$ with the adiabatic growing (resp. decaying) mode. A detailed and precise matching between the solutions (66), (67) -valid deep inside the radiation era- and the solution (71) -valid deep inside the matter era- would require some significant work in order to take into account various effects close to the time of equality. However, a crude matching is sufficient for catching the essential behavior of the matter power spectrum. From (66), (67) and (71) we obtain that at any time $\tau$ belonging to the MD stage,

$$
\delta_{\mathrm{m}}(k, \tau)=\left(4+\frac{k^{2} \tau^{2}}{3}\right) \frac{\delta_{\mathrm{m}}\left(k, \tau_{\mathrm{eq}}\right)}{4+k^{2} \tau_{\mathrm{eq}}^{2} / 3},
$$


where $\delta_{\mathrm{m}}\left(k, \tau_{\text {eq }}\right)$ is equal to Eq. (64) evaluated at $\tau=\tau_{\text {eq. }}$. Since we are interested only in modes which are inside the Hubble radius in the recent Universe, we can take the limit $k^{2} \tau^{2} \gg 1$. During matter domination, it is also possible to replace $\tau$ by $2 /(a H)$. Then, we obtain

$$
\delta_{\mathrm{m}}\left(k, \tau_{M}\right)=\frac{k^{2}}{3(a H)^{2}} \times \frac{\delta_{\mathrm{m}}\left(k, \tau_{\mathrm{eq}}\right)}{1+k^{2} \tau_{\mathrm{eq}}^{2} / 12} .
$$

\subsubsection{Dark energy domination}

After the matter dominated era, the dark energy component becomes the dominant contribution to the energy density of the Universe. In this era, the evolution of the gravitational potential is still given by Eq. (54), but now the effective mass term does not cancel. When the dark energy corresponds to the contribution of a cosmological constant with equation of state parameter $w=-1\left(P_{\Lambda}=-\rho_{\Lambda}\right)$,

$$
\ddot{\phi}+3 \frac{\dot{a}}{a} \dot{\phi}+\left(8 \pi G a^{2} \bar{\rho}_{\Lambda}\right) \phi=0
$$

and the potential decays proportionally to a scale-independent damping factor $g(\tau)=\phi(\tau) / \phi\left(\tau_{M}\right)$, where $\tau_{M}$ can be any time during matter domination. This factor can be found by numerical integration. In the case of a cosmological constant, a fairly good analytic approximation of $g\left(\tau_{0}\right)$ - where $\tau_{0}$ is the present time - is provided in Ref. [87]

$$
g\left(\tau_{0}\right) \simeq \Omega_{\mathrm{m}}^{0.2} /\left[1+0.003\left(\Omega_{\Lambda} / \Omega_{\mathrm{m}}\right)^{4 / 3}\right] .
$$

The modes probed by LSS experiments are all deep inside the Hubble radius during the end of matter domination and during $\Lambda$ domination. On those scales, the Poisson equation of Newtonian gravity (which corresponds to Eq. (52) with $k \gg a H$ ) gives a simple relation between the gravitational fluctuations and the matter perturbations,

$$
\Delta \phi=4 \pi G \bar{\rho}_{\mathrm{m}} \delta_{\mathrm{m}} \quad \Longleftrightarrow \quad \delta_{\mathrm{m}}=-\frac{k^{2} \phi}{4 \pi G a^{2} \bar{\rho}_{\mathrm{m}}}=-\frac{2 k^{2} \phi}{3 \Omega_{\mathrm{m}}(a H)^{2}}
$$

So, $\delta_{\mathrm{m}}$ is proportional to $a \phi$, which proves that the decay of the gravitational potential slows down the growth of matter fluctuations as a function of the scale factor. Also, we immediately obtain a relation between $\delta_{\mathrm{m}}$ during $\Lambda$ domination and matter domination

$$
\delta_{\mathrm{m}}(\tau)=\left[\frac{a\left(\tau_{M}\right) H\left(\tau_{M}\right)}{a(\tau) H(\tau)}\right]^{2} \frac{g(\tau)}{\Omega_{\mathrm{m}}(\tau)} \delta_{\mathrm{m}}\left(\tau_{M}\right)
$$


Finally, Eqs. (64), (72) and (77) give us the expression of matter perturbations on sub-Hubble scales during $\Lambda$ domination,

$$
\begin{aligned}
& \delta_{\mathrm{m}}(k, \tau)=\frac{g(\tau) k^{2} C(k)}{\Omega_{\mathrm{m}}(\tau)[a(\tau) H(\tau)]^{2}} \times \\
& \frac{\left[-(\sin k x) /(k x)+(\sin k x-k x \cos k x) /(k x)^{3}+\frac{1}{2}+\operatorname{Ci}(k x)-\mathcal{C}-\ln (k x)\right]}{\left[1+(k x)^{2} / 12\right]}
\end{aligned}
$$

where $x \equiv \tau_{\text {eq }} / \sqrt{3}$. The matter power spectrum is defined as $P(k, \tau)=$ $\left\langle\left|\delta_{\mathrm{m}}(k, \tau)\right|^{2}\right\rangle$. The previous equation allows us to relate it to the primordial power spectrum, usually parametrized by a power-law

$$
k^{3}\left\langle|\phi(k, \tau \rightarrow 0)|^{2}\right\rangle=\frac{k^{3}}{9}\left\langle|C(k)|^{2}\right\rangle \equiv A k^{n_{s}-1}
$$

where the index $n_{s}$ is the scalar tilt. We can compute the two asymptotes for the modes which were far outside/inside the sound horizon at the time of equality,

$$
\begin{array}{ll}
P(k, \tau) \simeq \frac{g(\tau)^{2}}{\Omega_{\mathrm{m}}(\tau)^{2}[a(\tau) H(\tau)]^{4}} \times \frac{A k^{n_{s}}}{4}, & k \tau_{\text {eq }} / \sqrt{3} \ll 1, \\
P(k, \tau) \simeq \frac{g(\tau)^{2}}{\Omega_{\mathrm{m}}(\tau)^{2}[a(\tau) H(\tau)]^{4}} \times \frac{9[12]^{2} A k^{n_{s}}[\ln k x]^{2}}{[k x]^{4}}, & k \tau_{\mathrm{eq}} / \sqrt{3} \gg 1 .
\end{array}
$$

Because of the crude approximations performed around the time of equality, this solution is not accurate, in particular for the global amplitude and for the behavior near $k \sim \sqrt{3} / \tau_{\text {eq }}$. However, the shape of the two asymptotes reflects the behavior of the true power spectrum obtained by a full numerical simulation, which is close to the BBKS [88] fitting formula

$$
\begin{aligned}
P(k, \tau) & \simeq \frac{g(\tau)^{2}}{\Omega_{\mathrm{m}}(\tau)^{2}[a(\tau) H(\tau)]^{4}} \frac{9 A k^{n_{s}}}{25} T(q)^{2} \\
T(q) & \equiv \frac{\ln (1+2.34 q)}{2.34 q}\left[1+3.89 q+(16.1 q)^{2}+(5.46 q)^{3}+(6.71 q)^{4}\right]^{-1 / 4} \\
q & \equiv \frac{k}{\Omega_{\mathrm{m}} h^{2} \exp \left(-2 \Omega_{b}\right) \mathrm{Mpc}} .
\end{aligned}
$$

Note also that the cosmological constant not only damps the perturbations $\delta \rho_{\mathrm{m}}$ but also implies a smaller fraction $\Omega_{\mathrm{m}}$. The net effect is that today and for fixed $a_{0} H_{0}$, the power spectrum normalization is enhanced by the cosmological constant (i.e. $g\left(\tau_{0}\right) / \Omega_{\mathrm{m}}\left(\tau_{0}\right)>1$ if $\Omega_{\Lambda}>0$ ). 


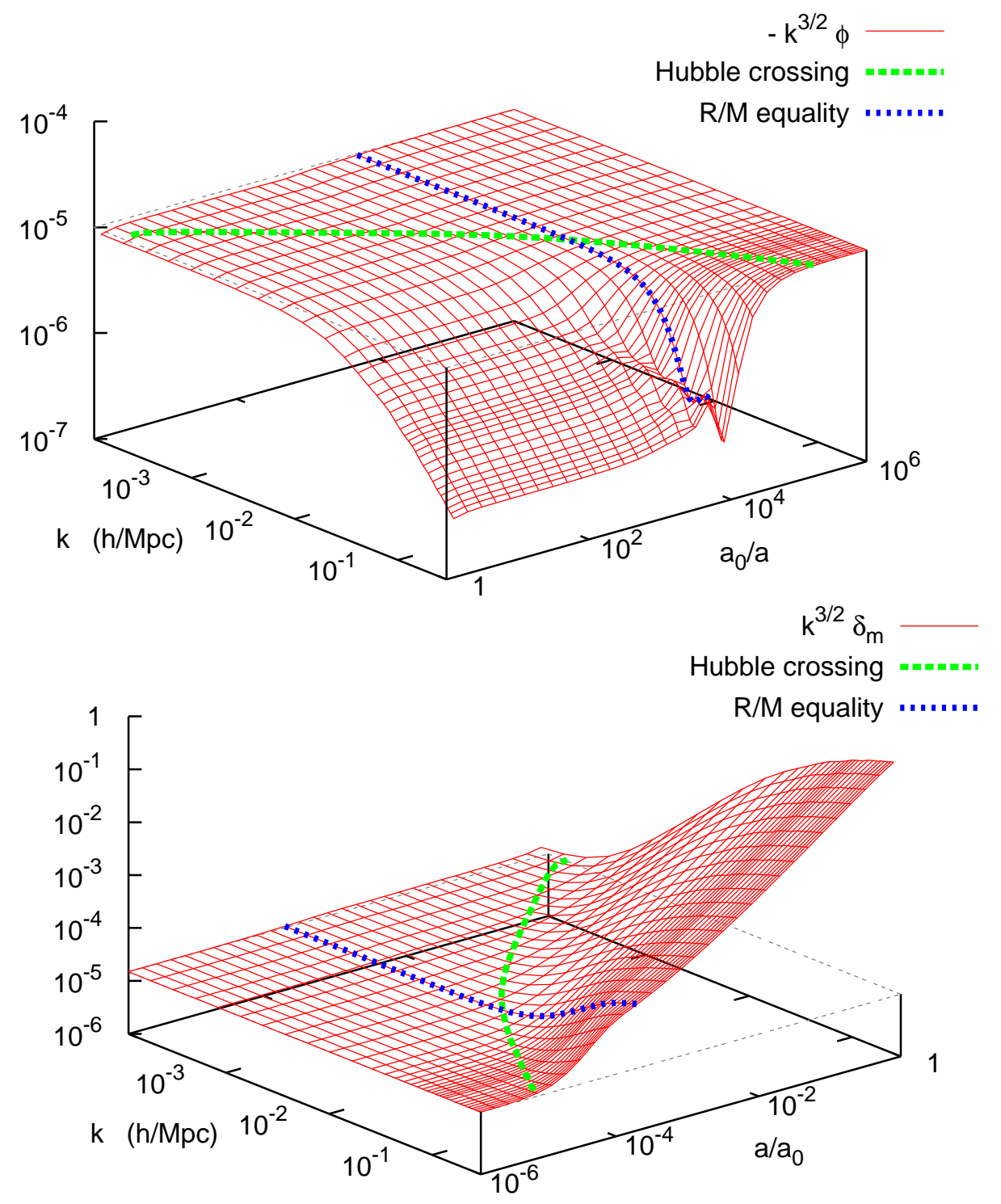

Fig. 6. Evolution of the longitudinal metric perturbations $\phi=\psi$ (top) and the matter density perturbations $\delta_{\mathrm{m}}=\delta \rho_{\mathrm{m}} / \bar{\rho}_{\mathrm{m}}$ (bottom) in a neutrinoless $\Lambda$ CDM model, obtained numerically with a Boltzmann code, as a function of the scale factor $a$ and Fourier wavenumber $k$. In order to get a better view, we chose opposite time axes in the two plots: time is evolving from back to front in the upper plot, and from front to back in the lower plot. The initial condition was set arbitrarily to $k^{3 / 2} \phi=-10^{-5}$.

\subsubsection{Numerical results}

We show in Fig. 6 the evolution of $\psi=\phi$ and $\delta_{\mathrm{m}}$ for a neutrinoless $\Lambda \mathrm{CDM}$ model, obtained numerically by solving the exact Einstein and conservation equations. We run the public code CMBFAST [49] based on the synchronous gauge, and convert the output into the longitudinal gauge variables used throughout this section. We start from a flat primordial spectrum with amplitude $k^{3 / 2} \phi=10^{-5}$ (let us recall that the "amplitudes" calculated by the code should be interpreted in the real Universe as the variance of random Gaussian 

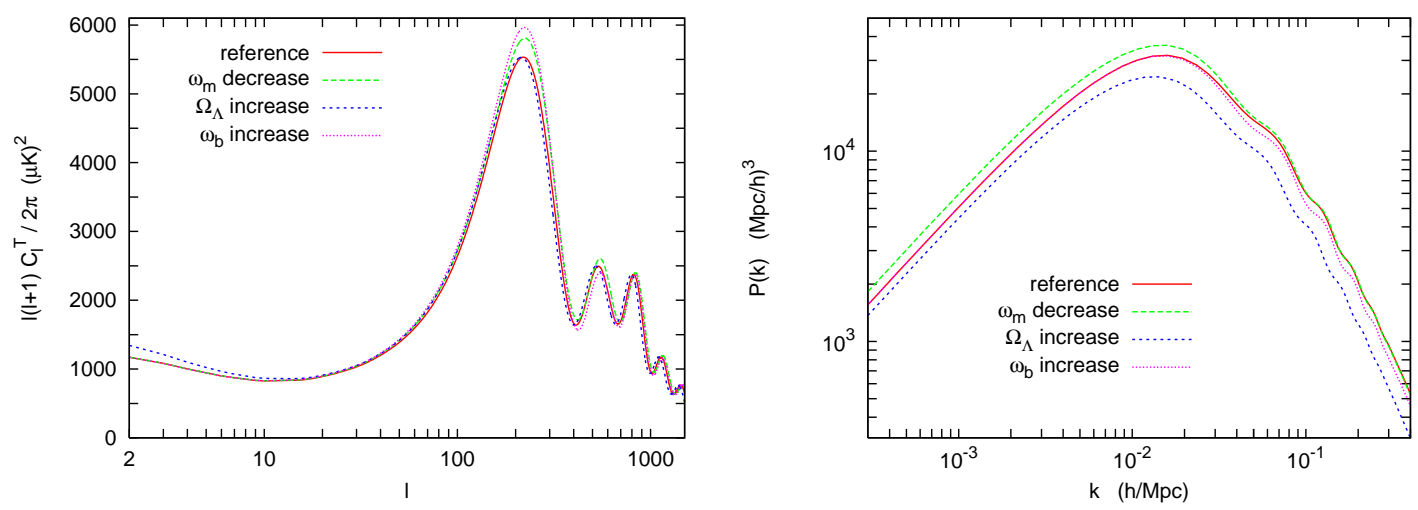

Fig. 7. (Red solid lines) The power spectrum of CMB temperature anisotropies in multipole space (left), and the LSS matter power spectrum evaluated today in Fourier space (right), for a reference neutrinoless $\Lambda$ CDM model with $\omega_{\mathrm{m}}=0.13$, $\Omega_{\Lambda}=0.74, \omega_{\mathrm{b}}=0.022, n_{s}=0.95, \tau=0.08$. (Dashed and dotted lines) Same power spectra for three models in which we keep all parameters fixed but one: either $\omega_{\mathrm{m}}=0.12$, or $\Omega_{\Lambda}=0.80$, or $\omega_{\mathrm{b}}=0.026$. The changes in the power spectra illustrate the effects labeled from (1) to (4) in Sec. 4.4.7, as explained in details at the end of this subsection.

perturbations). We show in Fig. 7 the CMB temperature anisotropy and LSS power spectra for the same cosmological model (but now with the primordial spectrum amplitude preferred by current data)

The global evolution is consistent with the analytical solutions found in the previous subsections. Indeed, for metric perturbations $\psi=\phi$ (upper plot, time evolving from back to front), we see that during radiation domination, super-Hubble modes are constant, and sub-Hubble modes are damped (with small oscillations). During matter domination, all modes are constant, so the shape imprinted near the time of equality survives until today (at least, as long as perturbations remain in the linear regime). During $\Lambda$ domination, there is a small scale-independent damping of all perturbations, hardly seen on the figure. For matter density perturbations $\delta_{\mathrm{m}}$ (lower plot, time evolving from front to back), we see that during radiation domination, super-Hubble modes are constant, and sub-Hubble modes grow slowly (asymptotically like the logarithm of $a$ ). During matter domination, super-Hubble modes are still constant, while sub-Hubble modes grow linearly with $a$. The smallest scales are the first ones to reach the condition of non-linearity $\delta_{\mathrm{m}} \sim 1$, and large scales are still linear today. During $\Lambda$ domination, $\delta_{\mathrm{m}}$ grows a bit more slowly as a function of $a$.

\subsubsection{Parameter dependence}

A minimal flat $\Lambda$ CDM models without neutrinos can be described with six parameters: the cosmological constant fraction $\Omega_{\Lambda}$, the total non-relativistic matter density $\omega_{\mathrm{m}}=\Omega_{\mathrm{m}} h^{2}$, the baryon density $\omega_{b}=\Omega_{b} h^{2}$, the primordial spectrum amplitude $A$ and tilt $n_{s}$, the optical depth to reionization $\tau$. These 
parameters control various physical effect which are responsible for the shape of the observable power spectra:

(1) The time of Radiation/Matter equality. In the above parameter basis, the time of equality between $\rho_{\mathrm{m}}$ and $\rho_{\mathrm{r}}$ is fixed by $\omega_{\mathrm{m}}$ only, since $\left(a_{\mathrm{eq}} / a_{0}\right)=\rho_{\mathrm{r}}^{0} / \rho_{\mathrm{m}}^{0}$ (the script ${ }^{0}$ means "evaluated today"), and $\rho_{\mathrm{r}}^{0}$ is fixed by the CMB temperature. A late equality implies more metric perturbation damping for modes entering the Hubble radius during radiation domination (see Fig. 6). For the LSS matter power spectrum, the consequence is the following: since on sub-Hubble scales $\delta_{\mathrm{m}}$ grows more efficiently during $\mathrm{MD}$ than during $\mathrm{RD}$ (because $\psi$ does not decay), it is clear that when $\omega_{\mathrm{m}}$ decreases (late equality) the matter power spectrum is suppressed on small scales relatively to large scales. Also, the global normalization increases, because of the factor $\omega_{\mathrm{m}}^{-2}$ in Eqs. $(80,81)$. So, the net effect of postponing equality is to amplifies $P(k)$ only for small $k$. For the CMB, the effect is opposite and counter-intuitive: small-scale perturbations are boosted. This is related to the dilation effect before recombination, and to the early Integrated Sachs-Wolfe effect just after recombination. In simple words, the rapid decay of $\psi(k, \tau)$ just after Hubble crossing during RD tends to boost the beginning of the first acoustic oscillation through gravitational redshift effects. So, a later equality induces higher CMB peaks, especially for the first one.

(2) The time of Matter $/ \Lambda$ equality. If the cosmological constant is larger, equality between matter and $\Lambda$ takes place earlier. We have seen that after that time, $\psi$ decays and $\delta_{\mathrm{m}}$ grows more slowly. Thus the matter power spectrum normalization is slightly suppressed by a long $\Lambda$ domination. The CMB spectrum is affected in a more subtle way: the time-variation of the metric perturbations leads to a net redshifting of the CMB photons traveling across a gravitational potential well at small $z$ (late Integrated Sachs-Wolfe effect). Therefore, the nearby distribution of galaxy cluster leaves an imprint in the large-scale CMB spectrum: the small- $l$ multipoles are enhanced (the larger is $\Lambda$, the more significant is the enhancement). This effect is difficult to measure experimentally due to cosmic variance (on large scales, we see only a few realization of the stochastic mode amplitudes, and we expect a large scattering of the data points around the theoretically predicted variance).

(3) The physical scale of the sound horizon at equality, and the angular scale of the sound horizon at recombination. From the previous sections it is clear that the characteristic scale in the matter power spectrum is fixed by the sound horizon at the time of equality (i.e., essentially, the Hubble scale $R_{H}\left(\tau_{\text {eq }}\right)$ divided by $\left.\sqrt{3}\right)$. Since this scale can be computed by extrapolating the present value of the Hubble parameter back in time, it depends on $h, \Omega_{\Lambda}$, and on the time of 
equality. In our parameter basis, it means that changing any of the two parameters $\omega_{\mathrm{m}}, \Omega_{\Lambda}$ shifts the matter power spectrum horizontally. The characteristic scale of the oscillations in the CMB power spectrum is set by the sound horizon $d_{s}\left(\tau_{\text {rec }}\right)$ at recombination. The time $\tau_{\text {rec }}$ is more or less fixed by thermodynamics, but the sound horizon is an integral over $c_{s} d t / a(t)$ between 0 and $\tau_{\text {rec }}$. This integral depends on the time of equality, and on the baryon density at late times (through $c_{s}$ ). In addition, the actual observable quantity is the angular scale of this sound horizon on the last scattering surface, which is given, for fixed $d_{s}\left(\tau_{\text {rec }}\right)$, by $h$ and $\Omega_{\Lambda}$. We conclude that the observed angular scale of the peaks constrains a combination of the three parameters $\Omega_{\Lambda}, \omega_{\mathrm{m}}$ and $\omega_{b}$.

(4) The balance between gravity and pressure in the tightly-coupled photon-baryon fluid. In this section, for simplicity, we assumed that before recombination the energy density of the tightly-coupled photonbaryon fluid was dominated by that of photons. Actually, when the baryon density becomes significant (near the time of equality), the fluid is more affected by the gravitational compression -which is more efficient for non-relativistic baryons than for relativistic photons- relatively to the competing photon pressure. Inside a gravitational potential well of given amplitude, the zero-point of oscillations (i.e. the instantaneous value of $\delta T / T$ for which gravity and pressure exactly cancel each other) is displaced. The main effect in the CMB spectrum is an increase of the first and third peaks, but not of the second one. As far as the matter power spectrum is concerned, we should remember that $P(k)$ probes the total contribution from baryonic and cold dark matter perturbations. In the limit of a small CDM contribution $\left(\omega_{b} / \omega_{\mathrm{m}} \rightarrow 1\right)$, the small-scale matter power spectrum has a low amplitude, because it does not benefit from the slow growth of CDM perturbations before equality on sub-Hubble scales (visible in Fig. 6). Also, in this limit, the oscillatory structure of $\delta_{b}$ at the time of recombination survives until now, and the matter power spectrum exhibits visible wiggles. In the opposite limit $\omega_{b} / \omega_{\mathrm{m}} \rightarrow 0$, which we adopted in our analytical approximate solution, the small-scale power spectrum is higher, and the baryonic oscillations in $P(k)$ are small (still, they are visible in Fig. 7 between $k=0.05 h^{-1} \mathrm{Mpc}$ and $k=0.2 h^{-1}$ $\mathrm{Mpc}$, and they have actually been observed recently, see Ref. [89]).

(5) The amplitude of primordial perturbations $(A)$, defined in Eq. (79), obviously fixes the global normalization of all spectra.

(6) The tilt of primordial perturbations $\left(n_{s}\right)$, defined in Eq. (79), fixes the balance between large and small scale amplitudes. Together with the previous parameter it provides interesting constraints on inflationary models. 
(7) The optical depth to reionization $(\tau)$ is a rather simplified way of parameterizing the reionization of the Universe at redshift of order 10 to 20 (depending on the model). This parameter has no effect on the matter power spectrum, but it damps the CMB temperature fluctuations for scales which are inside the Hubble horizon at the time of reionization, leaving small- $l$ temperature multipoles unchanged.

In Fig. 7, we illustrate graphically the consequence of varying either $\omega_{\mathrm{m}}, \Omega_{\Lambda}$ or $\omega_{\mathrm{b}}$, while keeping all the other cosmological parameters fixed. When we decrease $\omega_{\mathrm{m}}$, the relevant effects are (1) and (3): equality is postponed, boosting the first CMB peak as well as $P(k)$ for small $k$; simultaneously, there is a tiny horizontal shift of both spectra. When we increase $\Omega_{\Lambda}$, we can see the effects (2) and (3): the smallest CMB temperature multipoles increase, the $P(k)$ normalization decreases and both spectra are shifted to the left. When $\omega_{\mathrm{b}}$ is increased, the effects (3) and (4) are taking place: the first CMB peak increases, while the second one decreases slightly; $P(k)$ is slightly suppressed on small scales; finally, the scale of CMB peaks is slightly modified.

We have seen that $\left(A, n_{s}, \tau\right)$ have very specific effects, while $\left(\Omega_{\Lambda}, \omega_{\mathrm{m}}, \omega_{b}\right)$ have intricate effects. Nevertheless, since there are seven effects for six parameters, it is in principle easy to measure all of them from CMB and LSS data, assuming a flat $\Lambda$ CDM model. However, the neutrinoless model described in this section for pedagogical purposes is unable to provide a good fit to the data: it is time now to include neutrinos.

\subsection{Linear perturbation theory in presence of neutrinos ( $\Lambda M D M)$}

We will now restore the neutrino component, which is expected to behave at late times (after the non-relativistic transition) as an extra sub-dominant dark matter component: the standard cosmological scenario should actually be called $\Lambda$ Mixed Dark Matter $(\Lambda \mathrm{MDM})$.

The impact of neutrinos on cosmological perturbations was first clearly explained in 1980 by Bond, Efstathiou and Silk [70]. Later, during the 1980's, a very large number of papers investigated this issue in more details, and it would be difficult to provide an exhaustive list of references (see e.g. those given in $[6,90]$ ). Despite their interest, many of these papers are not of practical use nowadays because they generally assumed a neutrino-dominated universe with no CDM or $\Lambda$ components. For instance, the interested reader can take a look at Refs. [71,72] and go through the historical summary of Ref. [69].

Let us first introduce some notations and formalism -which are very close to those of Ma \& Bertschinger [74], but have been adapted to our metric signature $(+,-,-,-)$. The momentum of individual neutrinos can be labeled 
by $P_{i}$, which is the canonical conjugate of the comoving coordinate $x^{i}$. It differs from the proper momentum $p_{i}$ measured by a comoving observer (with fixed $x^{i}$ coordinates) by

$$
P_{i}=a(1-\psi) p_{i}
$$

Note that in absence of metric perturbations, $P_{i}$ would remain constant, while $p_{i}$ would decrease with the expansion like $a^{-1}$. Usually, the formulas are conveniently expressed in terms of the third variable $q_{i} \equiv a p_{i}$, which can be viewed as the proper momentum "corrected" from the effect of homogeneous expansion. The proper energy of a single neutrino measured by a comoving observer is equal to $\left(p^{2}+m^{2}\right)^{1 / 2}$. We define the related quantity $\epsilon$

$$
\epsilon=a\left(p^{2}+m^{2}\right)^{1 / 2}=\left(q^{2}+a^{2} m^{2}\right)^{1 / 2} .
$$

From $P_{\mu} P^{\mu}=m^{2}$ and $P_{i}=(1-\psi) q_{i}$, we get $P_{0}=(1+\phi) \epsilon$. Finally, we recall that by definition the phase-space distribution $f$ gives the number of particles in an infinitesimal phase-space volume: $d N=f\left(x^{i}, P_{j}, \tau\right) d^{3} x^{i} d^{3} P_{j}$.

\subsubsection{Collisionless fluids}

In the approximation of a homogeneous Universe, the phase-space distribution of neutrinos would be perfectly isotropic,

$$
f\left(x^{i}, P_{j}, \tau\right)=f_{0}(P, \tau)
$$

where $f_{0}$ is the Fermi-Dirac distribution already introduced in Eq. (6). Then, the energy-momentum tensor would be diagonal with an isotropic pressure term

$$
\begin{gathered}
T_{0}^{0}=\bar{\rho}_{\nu}=\frac{4 \pi}{a^{4}} \int q^{2} d q \epsilon f_{0}(q), \\
T_{i}^{i}=-\bar{p}_{\nu}=-\frac{4 \pi}{3 a^{4}} \int q^{2} d q \frac{q^{2}}{\epsilon} f_{0}(q) .
\end{gathered}
$$

Note that $f_{0}$ as a function of $q$ is time-independent,

$$
f_{0}(q)=\frac{1}{e^{q / a T_{\nu}}+1}
$$

because after neutrino decoupling the product of the neutrino temperature $T_{\nu}$ by the scale factor $a$ is a constant number. 
Spatial perturbations in the metric will induce variations in the neutrino phase-space distribution depending on time, space and momentum. The energymomentum tensor will be perturbed accordingly. With respect to a perfect fluid, the main difference is is that, in absence of microscopic interactions, there is no reason for the stress tensor $\delta T_{i j}$ to be isotropic at first order in perturbations. Therefore, the scalar sector of $\delta T_{\mu \nu}$ contains an extra degree of freedom, the anisotropic stress $\sigma$.

This can be understood in physical terms. First, strongly-interacting fluids have bulk motions: the coarse-grained fluid has a unique velocity in a given point. In the local rest-frame, an observer can measure the pressure e.g. by enclosing some particles in a small rubber balloon. At first order in perturbation, the balloon will expand or contract isotropically, because anisotropies arise from velocity gradients which can be shown to be second order in perturbations. In contrast, in collisionless fluids, there are no bulk motions: at a given point, there can be flows of particles in all directions. Inhomogeneities and anisotropies are described perturbatively by a function $\Psi \ll 1$ such that

$$
f\left(x^{i}, P_{j}, \tau\right)=f_{0}(q)\left[1+\Psi\left(x^{i}, q_{j}, \tau\right)\right] \quad \text { with } \quad P_{j}=(1-\Psi) q_{j},
$$

and anisotropic pressure can now appear at first order in perturbations. Indeed, the energy-momentum tensor can be computed from the phase-space distribution $f$ and the 4-momentum $P_{\mu}$

$$
T_{\mu \nu}=\int d P_{1} d P_{2} d P_{3}(-g)^{-1 / 2} \frac{P_{\mu} P_{\nu}}{P^{0}} f\left(x^{i}, P_{j}, \tau\right),
$$

where $(-g)^{-1 / 2}=a^{-4}(1-\phi+3 \psi)$. At first order in perturbations, this gives

$$
\begin{aligned}
& T_{0}^{0}\left(x^{i}\right)=a^{-4} \int q^{2} d q d \Omega \epsilon f_{0}(q)\left[1+\Psi\left(x^{i}, q \hat{n}_{j}, \tau\right)\right], \\
& T_{i}^{0}\left(x^{i}\right)=a^{-4} \int q^{2} d q d \Omega q \hat{n}_{i} f_{0}(q) \Psi\left(x^{i}, q \hat{n}_{j}, \tau\right), \\
& T_{j}^{i}\left(x^{i}\right)=-a^{-4} \int q^{2} d q d \Omega \frac{q^{2}}{\epsilon} \hat{n}_{i} \hat{n}_{j} f_{0}(q)\left[1+\Psi\left(x^{i}, q \hat{n}_{j}, \tau\right)\right],
\end{aligned}
$$

where $d \Omega$ is the differential of the momentum direction $\hat{n}_{j}=q_{j} / q$. It is then straightforward to obtain the perturbed components of the energy-momentum tensor

$$
\begin{aligned}
\delta \rho_{\nu} & =a^{-4} \int q^{2} d q d \Omega \epsilon f_{0} \Psi, & \delta P_{\nu} & =\frac{1}{3} a^{-4} \int q^{2} d q d \Omega \frac{q^{2}}{\epsilon} f_{0} \Psi, \\
\delta T_{i \nu}^{0} & =a^{-4} \int q^{2} d q d \Omega q \hat{n}_{i} f_{0} \Psi, & \Sigma_{j \nu}^{i} & =-a^{-4} \int q^{2} d q d \Omega \frac{q^{2}}{\epsilon}\left(\hat{n}_{i} \hat{n}_{j}-\frac{1}{3} \delta_{i j}\right) f_{0} \Psi .
\end{aligned}
$$




\subsubsection{Free-streaming}

We have seen that in perfect fluids, sound waves can propagate at the sound speed on scales smaller than the sound horizon. Sound waves cannot propagate in a collisionless fluid, but the individual particles free-stream with a characteristic velocity -for neutrinos, in average, the thermal velocity $v_{\text {th }}$. So, it is possible to define an horizon as the typical distance on which particles travel between time $t_{i}$ and $t$. During MD and RD and for $t \gg t_{i}$, this horizon is, as usual, asymptotically equal to $v_{\text {th }} / H$, up to a numerical factor of order one (see section 4.4.2). Exactly as we defined the Jeans length, we can define the free-streaming length by taking Eq. (48) and replacing $c_{s}$ by $v_{\text {th }}$

$$
k_{F S}(t)=\left(\frac{4 \pi G \bar{\rho}(t) a^{2}(t)}{v_{\mathrm{th}}^{2}(t)}\right)^{1 / 2}, \quad \lambda_{F S}(t)=2 \pi \frac{a(t)}{k_{F S}(t)}=2 \pi \sqrt{\frac{2}{3}} \frac{v_{\text {th }}(t)}{H(t)} .
$$

As long as neutrinos are relativistic, they travel at the speed of light and their free-streaming length is simply equal to the Hubble radius. When they become non-relativistic, their thermal velocity decays like

$$
v_{\text {th }} \equiv \frac{\langle p\rangle}{m} \simeq \frac{3 T_{\nu}}{m}=\frac{3 T_{\nu}^{0}}{m}\left(\frac{a_{0}}{a}\right) \simeq 150(1+z)\left(\frac{1 \mathrm{eV}}{m}\right) \mathrm{km} \mathrm{s}^{-1},
$$

where we used for the present neutrino temperature $T_{\nu}^{0} \simeq(4 / 11)^{1 / 3} T_{\gamma}^{0}$ and $T_{\gamma}^{0} \simeq 2.726 \mathrm{~K}$. This gives for the free-streaming wavelength and wavenumber during matter or $\Lambda$ domination

$$
\begin{aligned}
& \lambda_{F S}(t)=7.7 \frac{1+z}{\sqrt{\Omega_{\Lambda}+\Omega_{m}(1+z)^{3}}}\left(\frac{1 \mathrm{eV}}{m}\right) h^{-1} \mathrm{Mpc}, \\
& k_{F S}(t)=0.82 \frac{\sqrt{\Omega_{\Lambda}+\Omega_{m}(1+z)^{3}}}{(1+z)^{2}}\left(\frac{m}{1 \mathrm{eV}}\right) h \mathrm{Mpc}^{-1},
\end{aligned}
$$

where $\Omega_{\Lambda}$ (resp. $\Omega_{m}$ ) is the cosmological constant (resp. matter) density fraction evaluated today. So, after the non-relativistic transition and during matter domination, the free-streaming length continues to increase, but only like $(a H)^{-1} \propto t^{1 / 3}$, i.e. more slowly than the scale factor $a \propto t^{2 / 3}$. Therefore, the comoving free-streaming length $\lambda_{F S} / a$ actually decreases like $\left(a^{2} H\right)^{-1} \propto t^{-1 / 3}$. As a consequence, for neutrinos becoming non-relativistic during matter domination, the comoving free-streaming wavenumber passes through a minimum $k_{\mathrm{nr}}$ at the time of the transition, i.e. when $m=\langle p\rangle=3 T_{\nu}$ and $a_{0} / a=(1+z)=$ $2.0 \times 10^{3}(\mathrm{~m} / 1 \mathrm{eV})$. This minimum value is found to be

$$
k_{\mathrm{nr}} \simeq 0.018 \Omega_{\mathrm{m}}^{1 / 2}\left(\frac{m}{1 \mathrm{eV}}\right)^{1 / 2} h \mathrm{Mpc}^{-1} .
$$


The physical effect of free-streaming is to damp small-scale neutrino density fluctuations: neutrinos cannot be confined into (or kept outside of) regions smaller than the free-streaming length, for obvious kinematic reasons. We will see that the metric perturbations are also damped on those scales by gravitational back-reaction. On scales much larger than the free-streaming scale, the neutrino velocity can be effectively considered as vanishing, and after the non-relativistic transition the neutrino perturbations behave like cold dark matter perturbations. In particular, modes with $k<k_{\mathrm{nr}}$ are never affected by free-streaming and evolve like in a pure $\Lambda \mathrm{CDM}$ model.

\subsubsection{Vlasov equation}

The neutrino equation of evolution is the collisionless Boltzmann equation, or Vlasov equation, which just expresses the conservation of the number of particles along the phase-space trajectories

$$
\frac{D f}{d \tau}=\frac{\partial f}{\partial \tau}+\frac{d x^{i}}{d \tau} \frac{\partial f}{\partial x^{i}}+\frac{d q}{d \tau} \frac{\partial f}{\partial q}+\frac{d n_{i}}{d \tau} \frac{\partial f}{\partial n_{i}}=0 .
$$

We can expand $f$ like in Eq. (89) and keep only first-order perturbations. Using the fact that $d x^{i} / d \tau=P^{i} / P^{0}=-P_{i} / P_{0}=-q_{i} / \epsilon$ at order zero, that $d q / d \tau=$ $q \dot{\psi}+\epsilon \hat{n}_{i} \partial_{i} \phi$ at order one (from the geodesic equation $P^{0} \dot{P}^{0}+\Gamma_{\mu \nu}^{0} P^{\mu} P^{\nu}=0$ ), and that $d n_{i} / d \tau=0$ at order zero (in absence of metric perturbations, the momenta keep a fixed orientation), we obtain

$$
f_{0} \dot{\Psi}-\frac{q_{i}}{\epsilon} f_{0} \partial_{i} \Psi+\left(q \dot{\psi}+\epsilon \hat{n}_{i} \partial_{i} \phi\right) \frac{\partial f_{0}}{\partial q}=0
$$

or in Fourier space and after dividing by $f_{0}$

$$
\dot{\Psi}-i \frac{q}{\epsilon}(\vec{k} \cdot \hat{n}) \Psi=-\left(\dot{\psi}+i \frac{\epsilon}{q}(\vec{k} \cdot \hat{n}) \phi\right) \frac{\partial \ln f_{0}}{\partial \ln q} .
$$

This equation gives the response of the neutrino phase-space distribution to metric perturbations.

\subsubsection{Neutrino perturbations during the relativistic regime}

When neutrinos are ultra-relativistic $(\epsilon=q)$, the Vlasov equation reduces to

$$
\dot{\Psi}-i(\vec{k} \cdot \hat{n}) \Psi=-(\dot{\psi}+i(\vec{k} \cdot \hat{n}) \phi) \frac{\partial \ln f_{0}}{\partial \ln q}
$$


The homogeneous equation does not involve $q$, while the source term has a definite $q$-dependence, fixed once and for all by the shape of the homogeneous distribution. This simply reflects the fact that neutrinos at a given point and in a given direction are all redshifted or blueshifted by the same amount, so that their spectrum always remain that of a blackbody, with a temperature shift $\delta T_{\nu}\left(x^{i}, \hat{n}_{j}, \tau\right)$ at a given point and in a given direction

$$
\begin{aligned}
f\left(x^{i}, q, \hat{n}_{j}, \tau\right) & =\left(\exp \left[\frac{q}{a\left(T_{\nu}+\delta T_{\nu}\right)}\right]+1\right)^{-1} \\
& =f_{0}(q)+\frac{\partial f_{0}}{\partial T_{\nu}}(q) \delta T_{\nu}\left(x^{i}, \hat{n}_{j}, \tau\right) .
\end{aligned}
$$

Since $f_{0}$ is a function of $q / a T_{\nu}$, one has $\partial f_{0} / \partial T_{\nu}=-\left(q / T_{\nu}\right) \partial f_{0} / \partial q$, and

$$
f\left(x^{i}, q, \hat{n}_{j}, \tau\right)=f_{0}(q)-\frac{\partial f_{0}}{\partial \ln q}(q) \frac{\delta T_{\nu}}{T_{\nu}}\left(x^{i}, \hat{n}_{j}, \tau\right) .
$$

Identifying this result with the definition of the phase-space perturbation $\Psi$, we get

$$
\Psi\left(x^{i}, q, \hat{n}_{j}, \tau\right)=-\frac{\partial \ln f_{0}}{\partial \ln q}(q) \frac{\delta T_{\nu}}{T_{\nu}}\left(x^{i}, \hat{n}_{j}, \tau\right) .
$$

We now see explicitly that the presence of the function $\frac{\partial \ln f_{0}}{\partial \ln q}$ in the Vlasov equation means precisely that the spectrum remains Planckian. Since for relativistic neutrinos the local $q$-dependence is trivial, we only need to compute the evolution of the mean momentum, which is a function of position, direction and time. One can define the quantity $F_{\nu}\left(x^{i}, \hat{n}_{j}, \tau\right)$ as

$$
F_{\nu}\left(x^{i}, \hat{n}_{j}, \tau\right) \equiv \frac{\int q^{2} d q q f_{0}(q)\left[1+\Psi\left(x^{i}, \hat{n}_{j}, \tau\right)\right]}{\int q^{2} d q q f_{0}(q)}-1
$$

for which the Vlasov equation reads in Fourier space (after integrating by part)

$$
\dot{F}_{\nu}-i(\vec{k} \cdot \hat{n}) F_{\nu}=4(\dot{\psi}+i(\vec{k} \cdot \hat{n}) \phi) .
$$

We can further reduce the dimensionality of the problem by noticing that the evolution depends only on the direction $\hat{n}$ through the angle $\vec{k} \cdot \hat{n}$, which is a natural consequence of the isotropy of the homogeneous background. We perform a Legendre expansion with respect to this angle,

$$
F_{\nu}(\vec{k}, \hat{n}, \tau)=\sum_{l=0}^{\infty}(-i)^{l}(2 l+1) F_{\nu l}(\vec{k}, \tau) P_{l}(\hat{k} \cdot \hat{n}) .
$$


By comparing Eqs. (92) and (93) (with $\epsilon=q$ ) with the above definitions, it is straightforward to show that the density contrast (as well as $\delta p_{\nu}=\frac{1}{3} \delta \rho_{\nu}$ ) is given by the monopole: $\delta_{\nu}=F_{\nu 0}$, the velocity gradient by the dipole: $\theta_{\nu}=$ $\frac{3}{4} k F_{\nu 1}$, and the shear (or anisotropic stress) by the quadrupole: $\sigma_{\nu}=-\frac{1}{2} F_{\nu 2}$. The Vlasov equation now reduces to its most "concentrated" form: an infinite hierarchy of coupled equation for the multipoles $F_{\nu l}(\vec{k}, \tau)$

$$
\begin{aligned}
\dot{\delta}_{\nu} & =\frac{4}{3} \theta_{\nu}+4 \dot{\psi}, \\
\dot{\theta}_{\nu} & =-\frac{k^{2}}{4} \delta_{\nu}-k^{2} \sigma_{\nu}-k^{2} \phi, \\
\dot{F}_{\nu l} & =\frac{k}{2 l+1}\left[(l+1) F_{\nu(l+1)}-l F_{\nu(l-1)}\right], \quad l \geq 2 .
\end{aligned}
$$

Note that the first two equations consistently reproduce the continuity and Euler equations (39)-(40).

After introducing all this formalism, it is possible to understand the physical evolution of relativistic neutrino perturbations. We assume isentropic (adiabatic) initial conditions during radiation domination and on super-Hubble scales: so, we start from $\delta_{\nu}=\delta_{\gamma}$ and from time-independent metric fluctuations. On super-Hubble scales, the neutrino distribution is static, with a local density (and temperature) perturbation proportional to the metric fluctuation. An observer would see locally a quasi-isotropic temperature distribution: the dipole is suppressed by a factor $k / a H=k \tau$, and the $l$-th multipole by $(k \tau)^{l}$. At leading order in $(k \tau)$, the Einstein equations (35) and (36) immediately give

$$
\delta_{\nu}=\delta_{\gamma}=-2 \phi=4 \frac{\delta T_{\nu}}{T_{\nu}}, \quad \theta_{\nu}=\theta_{\gamma}=-\frac{1}{2} k^{2} \tau \phi
$$

while the expressions of $\psi$ and $\sigma_{\nu}$ require more work (actually, the usual way to obtain them is to solve the Einstein equations in the long wavelength limit in the synchronous gauge, and then to convert the result into longitudinal gauge variables). The result is found to be (see Ref. [74])

$$
\psi=\left(1+\frac{2}{5} R_{\nu}\right) \phi, \quad \sigma_{\nu}=-\frac{1}{15}(k \tau)^{2} \phi, \quad \text { with } \quad R_{\nu} \equiv \frac{\bar{\rho}_{\nu}}{\bar{\rho}_{\nu}+\bar{\rho}_{\gamma}} .
$$

For three standard non-relativistic neutrinos, on has $R_{\nu} \simeq 0.405$ and $\psi \simeq$ $1.162 \phi$. After Hubble crossing, i.e. on scales smaller than the free-streaming length, the various multipoles are populated one after each other, at the expense of the low multipoles which get reduced. Physically, this corresponds to the fact that an observer would see locally the superposition of a growing number of neutrino flows, coming from all directions. Since the flows come from 

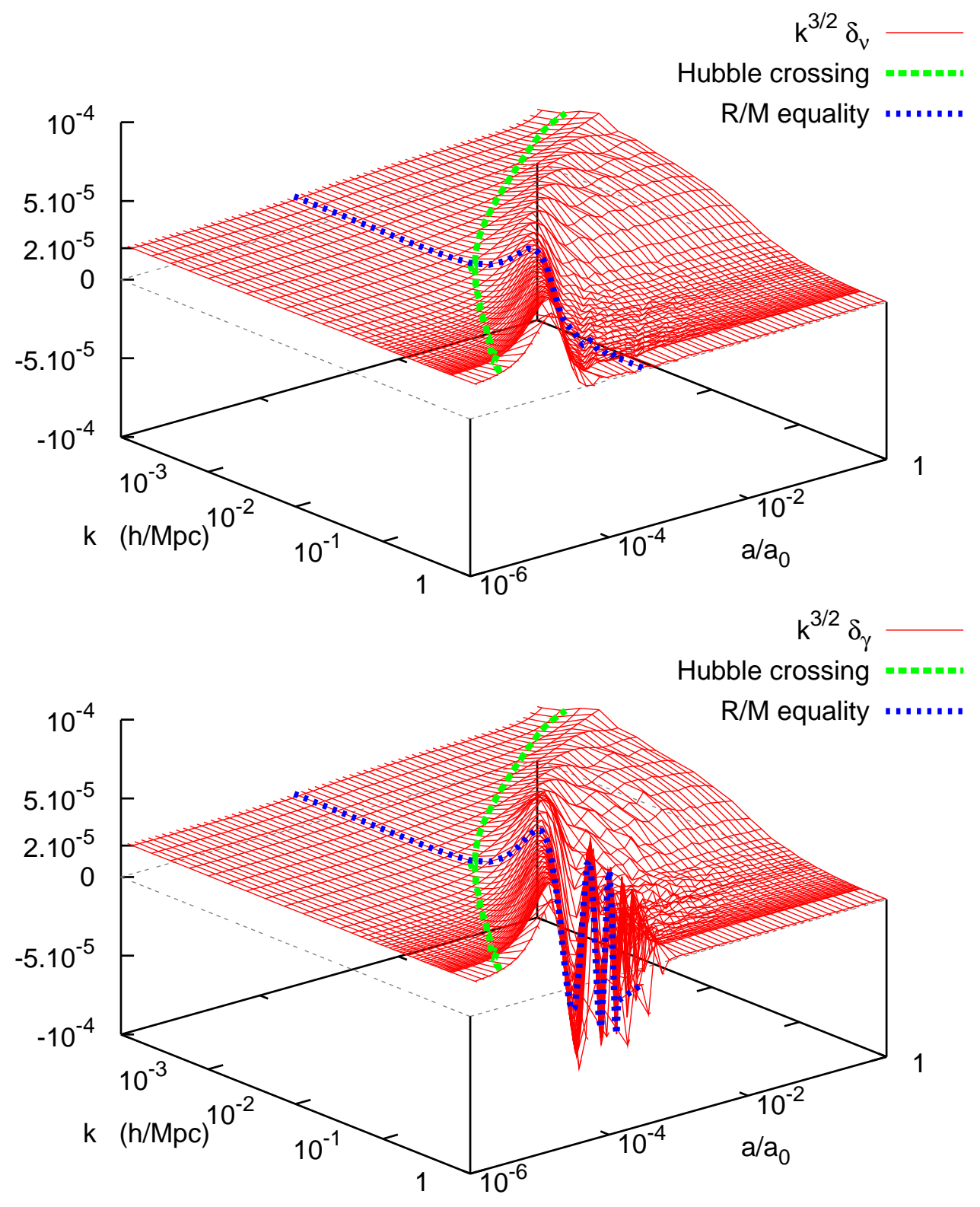

Fig. 8. Evolution of (longitudinal gauge) massless neutrino density perturbations $\delta_{\nu}=\delta \rho_{\nu} / \bar{\rho}_{\nu}$ (top), compared with those of the photon density $\delta_{\gamma}=\delta \rho_{\gamma} / \bar{\rho}_{\gamma}$ (bottom), in a $\Lambda$ CDM model with three massless neutrinos, as a function of the scale factor $a$ and Fourier wavenumber $k$, obtained numerically with a Boltzmann code. Time is evolving from left $\left(a / a_{0}=10^{-6}\right)$ to right $\left(a / a_{0}=1\right)$, and the initial condition is set arbitrarily to $k^{3 / 2} \phi=-10^{-5}$. Unlike the photon density contrast, which oscillates between Hubble crossing and equality, the neutrino density contrast is immediately damped after the first half-oscillation. After equality, $\delta_{\nu}$ and $\delta_{\gamma}$ remain constant: they are preserved from gravitational collapse by free-streaming and relativistic pressure.

a random distribution of over- and under-densities, the local density contrast tends to average to zero. 


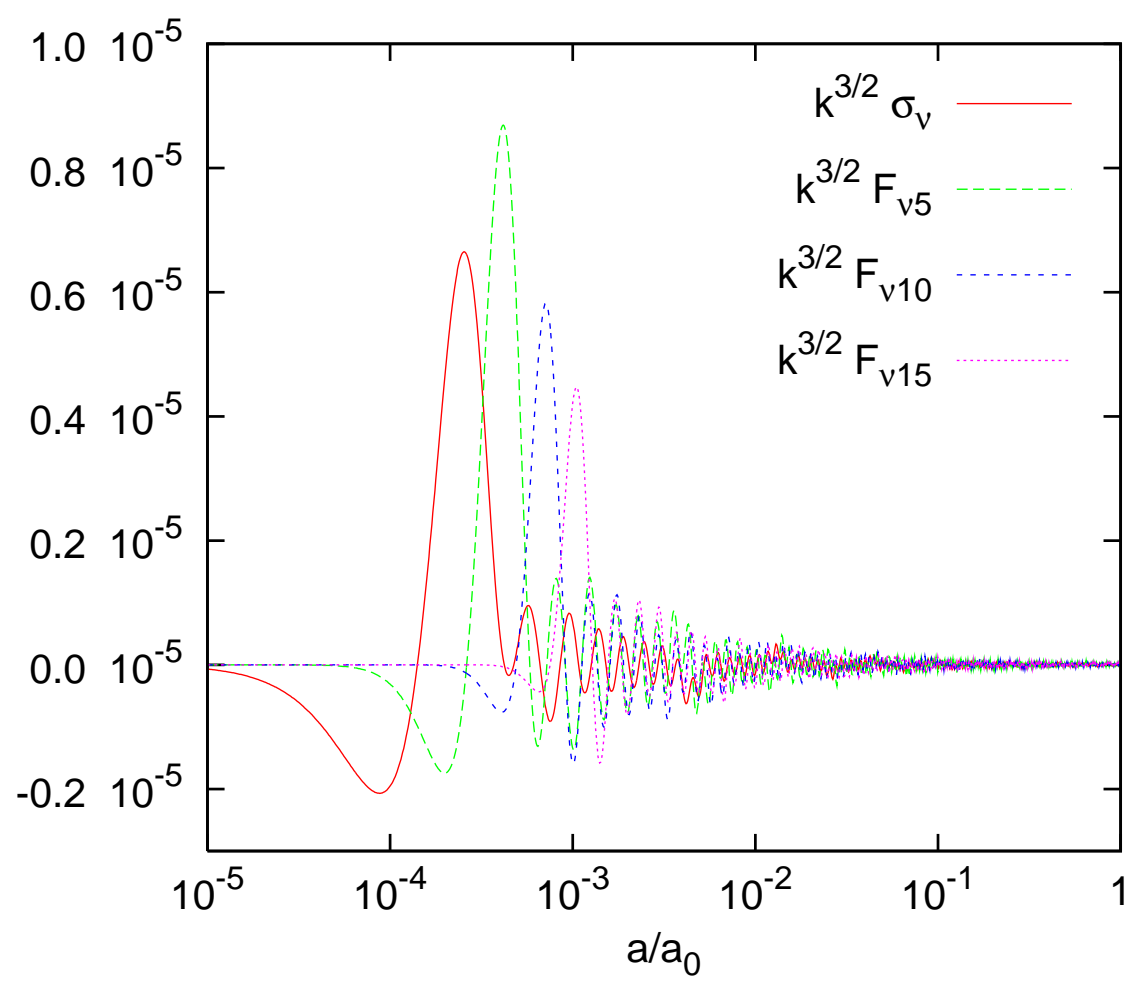

Fig. 9. Evolution of some of the first neutrino multipoles $F_{\nu l}$ as a function of the scale factor, for the mode $k=0.1 \mathrm{~h} \mathrm{Mpc}^{-1}$ which enters into the Hubble radius close to the end of radiation domination, when $a / a_{0}=2.5 \times 10^{-5}$. We imposed an initial condition $k^{3 / 2} \phi=-10^{-5}$. We see that after Hubble crossing, the multipoles are populated one after each other, reflecting the fact that an observer would see locally a superposition of a growing number of neutrino flows, coming from all directions.

It is useful for pedagogical purpose to compare the evolution equation of neutrinos with that of photons before recombination. For this purpose, we combine the continuity and Euler equations into a second-order equation

$$
\ddot{\delta}_{\nu}=-\frac{1}{3} k^{2} \delta_{\nu}-\frac{4}{3} k^{2} \phi+4 \ddot{\psi}-\frac{4}{3} k^{2} \sigma_{\nu}
$$

In absence of shear, this equation would be similar to the photon equation of evolution before recombination and in the limit of small baryon-to-photon ratio $R_{b}=\bar{\rho}_{b} / \bar{\rho}_{\gamma}$. The terms are easy to identify: $-\frac{1}{3} k^{2} \delta_{\nu}$ is the force arising from relativistic pressure, $-\frac{4}{3} k^{2} \phi$ the gravitational force, and $4 \ddot{\psi}$ the dilation effect: a local increase of $\psi$ is equivalent to a local decrease of the scale factor and of the photon/neutrino wavelength, and hence to an increase of the blackbody temperature and density. In the case of photons, in the tight-coupling limit, the microscopic interactions guarantee that $\sigma_{\gamma}$ vanishes (even if $R_{b} \ll 1$ ), and the equivalent of the above equation is that of an harmonic oscillator driven by metric fluctuations. In the case of neutrinos, the shear becomes important 
inside the Hubble length, and couples the above equation to the whole multipole hierarchy; qualitatively, the shear acts like viscosity in a fluid; instead of experiencing driven oscillations, the neutrino overdensity $\delta_{\nu}$ is damped, while the power is transfered to higher multipoles. This behavior is illustrated in Figs. 8 and 9 which derive from exact numerical simulations (using the code CMBFAST [49]). In particular, one can see explicitly on Fig. 8 that in the region where the photon density contrast experiences acoustic oscillations, the neutrino density contrasts is damped.

It is possible to gain even more intuition by going back to the relativistic Vlasov equation (101), for which the full solution can be decomposed into those of the homogeneous and inhomogeneous differential equations. The general solution of the homogeneous equation is actually trivial

$$
\Psi(\vec{k}, \hat{n}, q, \tau)=-\frac{\delta T_{\nu}}{T_{\nu}}(\vec{k}, \hat{n}) e^{i(\vec{k} \cdot \hat{n}) \tau} \frac{\partial \ln f_{0}}{\partial \ln q}
$$

It corresponds to the free propagation of plane waves of temperature perturbations, i.e. to free-streaming. In the $F_{\nu l}$ basis, this yields $F_{\nu l}(\vec{k}, \tau) \propto j_{l}(k \tau)$, where the $j_{l}$ 's are the spherical Bessel functions of the first kind, and the contribution to $\delta_{\nu}$ is equal to $[\sin (k \tau) /(k \tau)]$ times a constant of integration. As expected, this contribution tends to zero after Hubble crossing (because of the averaging between various flows), while higher multipoles are populated one after each other, with a peak around $\tau \sim l / k$ (the numerical solution of Fig. 9 looks slightly more complicated than plain Bessel functions: this is due to the small time-variation of $\phi$ and $\psi$ caused by neutrino shear). On top of these terms, the full solution receives a contribution from the solution of the inhomogeneous equation. During radiation domination, $\psi$ and $\phi$ decay, and this solution is complicated. However, it is clear that it should also decay. Instead, during matter domination, $\psi$ and $\phi$ are frozen, and the solution is obvious: $\Psi=\phi \frac{\partial \ln f_{0}}{\partial \ln q}$. In the $F_{\nu l}$ basis, this term only contributes to $F_{\nu 0}=\delta_{\nu}$, for which the full solution reads

$$
\delta_{\nu}=-4 \phi(k)+\alpha(k) \sin (k \tau) /(k \tau), \quad \tau>\tau_{\mathrm{eq}},
$$

where the constant of integration $\alpha(k)$ could be found my matching with the radiation-dominated solution. The first term corresponds to the stationary equilibrium configuration for which, in any potential well, the relativistic pressure compensates exactly the gravitational force. This behavior can be clearly seen on Fig. 8 (note that after decoupling, the photons free-stream like the relativistic neutrinos, so $\delta_{\gamma}$ also tends towards $\left.-4 \phi\right)$.

In conclusion, during the relativistic regime, the combined effects of freestreaming and relativistic pressure result in a suppression of the neutrino density contrast inside the Hubble radius: during radiation domination, $\delta_{\nu}$ is 
driven to zero instead of oscillating like $\delta_{\gamma}$ and $\delta_{\mathrm{b}}$, and during matter domination, it remains constant instead of growing like $\delta_{\mathrm{b}}$ and $\delta_{\mathrm{cdm}}$.

\subsubsection{Neutrino perturbations during the non-relativistic regime}

After the non-relativistic transition, the $q$-dependence of the phase-space distribution becomes non-trivial. The geodesic equation

$$
\frac{d q}{d \tau}=q \dot{\psi}+\left(q^{2}+a^{2} m^{2}\right)^{1 / 2} \hat{n}_{i} \partial_{i} \phi
$$

shows that the amount of blueshift or redshift $d q / q$ experienced by a neutrino when it travels over metric fluctuations depends on how non-relativistic it is. In a given point and direction $\left(x^{i}, \hat{n}_{j}\right)$, there is a superposition of neutrinos of different momenta which are more or less deep inside the non-relativistic regime. Lowest and highest energy neutrinos experience different redshifts, and the thermal spectrum of Eqs. (102) and (103) is inevitably distorted at first order in perturbation (but not at zero order, since the distortion is induced by metric perturbations and not by the background geometry). Therefore, it is impossible to eliminate the $q$-dependence from the problem, as we did in the relativistic regime. However, it is still possible to get rid partially of the direction dependence by expanding $\Psi$ in multipole space

$$
\Psi(\vec{k}, q, \hat{n}, \tau)=\sum_{l=0}^{\infty}(-i)^{l}(2 l+1) \Psi_{l}(\vec{k}, q, \tau) P_{l}(\hat{k} \cdot \hat{n})
$$

Then, the Vlasov equation becomes

$$
\begin{aligned}
& \dot{\Psi}_{0}=\frac{q k}{\epsilon} \Psi_{1}-\dot{\psi} \frac{\partial \ln f_{0}}{\partial \ln q}, \\
& \dot{\Psi}_{1}=\frac{q k}{3 \epsilon}\left(\Psi_{2}-2 \Psi_{0}\right)+\frac{\epsilon k}{3 q} \phi \frac{\partial \ln f_{0}}{\partial \ln q}, \\
& \dot{\Psi}_{l}=\frac{q k}{(2 l+1) \epsilon}\left[(l+1) \Psi_{l+1}-l \Psi_{l-1}\right], \quad l \geq 2 .
\end{aligned}
$$

The perturbed energy density, pressure, energy flux divergence and shear stress in $k$-space are given by

$$
\begin{aligned}
& \delta \rho_{\nu}=4 \pi a^{-4} \int q^{2} d q \epsilon f_{0}(q) \Psi_{0}, \\
& \delta p_{\nu}=\frac{4 \pi}{3} a^{-4} \int q^{2} d q \frac{q^{2}}{\epsilon} f_{0}(q) \Psi_{0},
\end{aligned}
$$




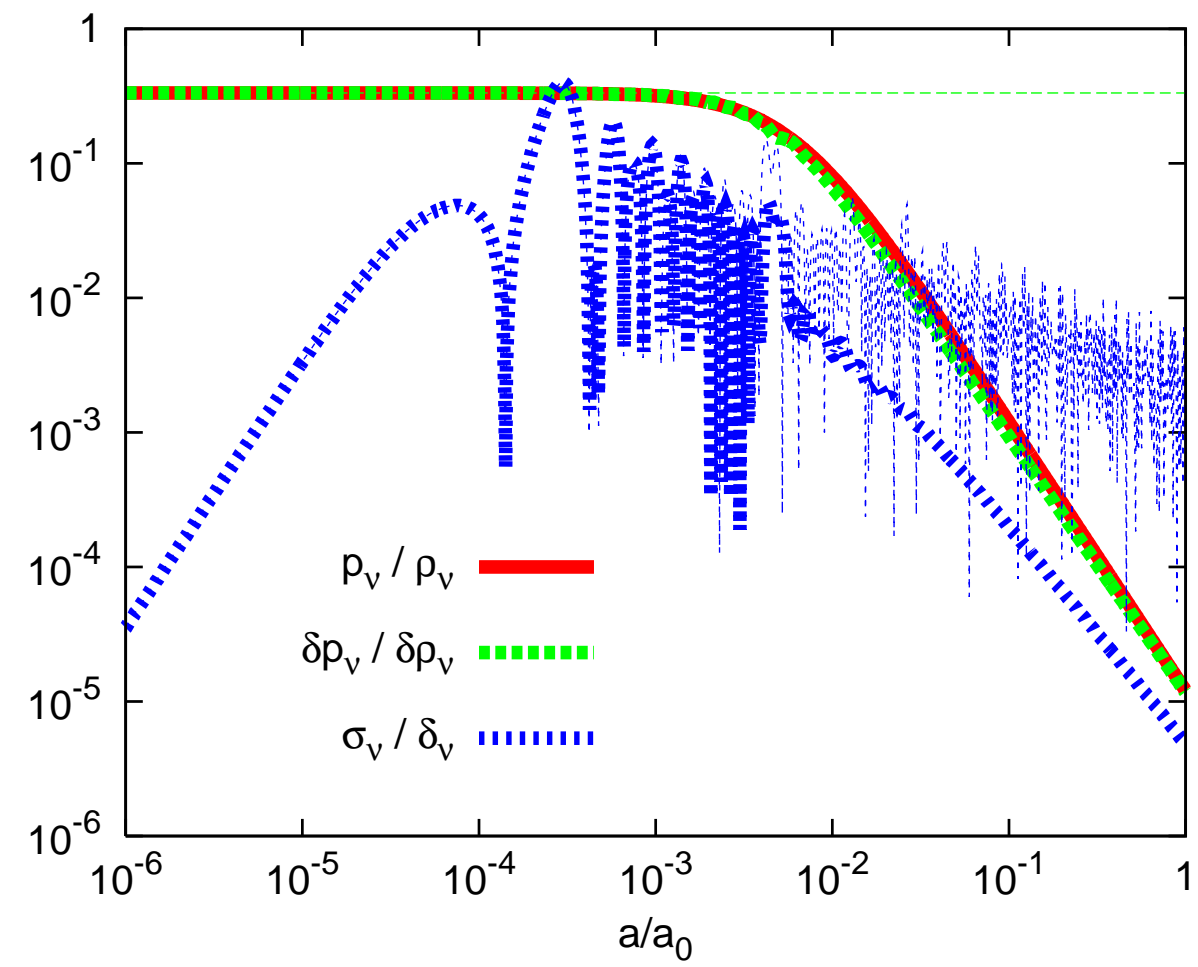

Fig. 10. Evolution of the neutrino isotropic pressure perturbation $\delta p_{\nu}$ and anisotropic stress $\sigma_{\nu}$ in units of the density perturbation (for the mode $k=0.1 \mathrm{~h} \mathrm{Mpc}^{-1}$ which enters into the Hubble radius when $a / a_{0}=2.5 \times 10^{-5}$ ) compared to the evolution of the background equation of state parameter $\bar{p}_{\nu} / \bar{\rho}_{\nu}$. The thin curves show the case of massless neutrinos for which $\bar{p}_{\nu} / \bar{\rho}_{\nu}=\delta p_{\nu} / \delta \rho_{\nu}=1 / 3$ and $\sigma_{\nu} / \delta_{\nu}$ decays slowly after Hubble crossing. The thick lines correspond to neutrinos with $m_{\nu}=0.1 \mathrm{eV}$, which become non-relativistic around $a / a_{0}=5 \times 10^{-3}$. After the transition, pressure and shear perturbations become negligible with respect to density perturbations, just like for cold dark matter.

$$
\begin{aligned}
& \left(\bar{\rho}_{\nu}+\bar{p}_{\nu}\right) \theta_{\nu}=4 \pi k a^{-4} \int q^{2} d q q f_{0}(q) \Psi_{1}, \\
& \left(\bar{\rho}_{\nu}+\bar{p}_{\nu}\right) \sigma_{\nu}=-\frac{8 \pi}{3} a^{-4} \int q^{2} d q \frac{q^{2}}{\epsilon} f_{0}(q) \Psi_{2} .
\end{aligned}
$$

When for a given family the neutrinos are well inside the non-relativistic regime, i.e. when most of the momenta for which $f_{0}(q)$ is non-negligible satisfy $q \ll \epsilon \sim a m$, the above equations show that both $\delta p_{\nu}$ and $\left(\bar{\rho}_{\nu}+\bar{p}_{\nu}\right) \sigma_{\nu}$ are suppressed with respect to $\delta \rho_{\nu}$ (as can be checked in Fig. (10)). As expected, the continuity and Euler equations (39) and (40) become gradually identical to those of ordinary non-relativistic matter

$$
\begin{aligned}
& \dot{\delta}_{\nu}=\theta_{\nu}+3 \dot{\psi}, \\
& \dot{\theta}_{\nu}=-\frac{\dot{a}}{a} \theta_{\nu}-k^{2} \phi .
\end{aligned}
$$


Like for CDM (and baryons after recombination), this system leads to the second-order equation of evolution

$$
\ddot{\delta}_{\nu}+\frac{\dot{a}}{a} \dot{\delta}_{\nu}=-k^{2} \phi+3\left(\ddot{\psi}+\frac{\dot{a}}{a} \dot{\psi}\right)
$$

We are interested in neutrinos becoming non-relativistic during matter domination, when $\phi$ and $\psi$ are approximately constant and $a \propto \tau^{2}$. In this case, the solution of (120) reads

$$
\begin{aligned}
\delta_{\nu} & =A \ln \tau+B-\frac{(k \tau)^{2}}{6} \phi \\
& =\tilde{A} \ln a+\tilde{B}-\frac{2}{3}\left(\frac{k}{a H}\right)^{2} \phi,
\end{aligned}
$$

where $A, B$ (or $\tilde{A}, \tilde{B})$ are some constants of integration. The last term (the solution of the inhomogeneous equation) grows like $\tau^{2}$, i.e. like the scale factor. This solution has a simple interpretation:

- first, for $k>k_{\mathrm{nr}}$, i.e. for modes which are inside the Hubble radius at the time of the non-relativistic transition, the density contrast $\delta_{\nu}$ is much smaller than $\delta_{\text {cdm }}$ at that time (as a consequence of free streaming), and then grows faster than $\delta_{\text {cdm }} \propto a$ because of the term $(\tilde{A} \ln a)$. At some point the first and third terms in Eq. (122) will be equal, and then asymptotically $\delta_{\nu}$ will be dominated by

$$
\delta_{\nu} \longrightarrow-\frac{2}{3}\left(\frac{k}{a H}\right)^{2} \phi \propto a,
$$

which corresponds exactly to the solution of the Poisson equation in a matter-dominated Universe (see Eq. (76) with $\Omega_{\mathrm{m}}=1$ ). Indeed, this asymptotic value is simply the one imposed to all non-relativistic species by Newtonian gravitation, and after an infinite amount of time $\delta_{\nu}$ would be equal to $\delta_{\text {cdm }}$ on all scales. The same is also true for baryons after photon decoupling, but since baryons did not experience free-streaming, the equality between $\delta_{\mathrm{b}}$ and $\delta_{\mathrm{cdm}}$ is reached quickly after $\tau=\tau_{\mathrm{dec}}$. Instead, for neutrinos with masses of order of $1 \mathrm{eV}$ or smaller, the asymptote is far from being reached today, except for scales very close to $k=k_{\mathrm{nr}}$. On smaller scales, $\delta_{\nu}$ remains much smaller than $\delta_{\mathrm{cdm}} \simeq \delta_{\mathrm{b}}$.

- second, for $k<k_{\mathrm{nr}}$, i.e. for modes which are outside the Hubble radius at the time of the non-relativistic transition, the density contrast $\delta_{\nu}$ is timeindependent and of the same order as $\left(\phi, \delta_{\mathrm{cdm}}\right)$ on super-Hubble scales. After horizon crossing, the solution is dominated again by (123) and becomes rapidly equal to $\delta_{\mathrm{cdm}}$. 
The conclusion of this section is that if we could picture the three density contrasts at a fixed time near the end of matter domination, we would expect $\delta_{\nu}=\delta_{\text {cdm }}=\delta_{\mathrm{b}}$ for $a_{0} H_{0}<k<k_{\mathrm{nr}}$, while for $k>k_{\mathrm{nr}}$ the ratio $\delta_{\nu} / \delta_{\mathrm{cdm}}=\delta_{\nu} / \delta_{\mathrm{b}}$ would be smaller than one, and tending towards zero as $k$ goes to infinity. The recent stage of $\Lambda$ domination does not change this conclusion: for $a_{0} H_{0}<$ $k<k_{\mathrm{nr}}$, the three density contrasts are damped by the same amount, and on smaller scales the ratio $\delta_{\nu} / \delta_{\text {cdm }}$ does not change significantly. All these features can be checked in the numerical simulations presented in Sec. 4.5.7 (see Fig. 11). Therefore, the matter power spectrum today (or at any time after the non-relativistic transition of the heaviest neutrinos) is given by

$$
\begin{aligned}
& P(k)=\left\langle\left(\frac{\delta \rho_{\mathrm{cdm}}+\delta \rho_{\mathrm{b}}+\delta \rho_{\nu}}{\rho_{\mathrm{cdm}}+\rho_{\mathrm{b}}+\rho_{\nu}}\right)^{2}\right\rangle \\
& =\left\langle\left(\frac{\Omega_{\mathrm{cdm}} \delta_{\mathrm{cdm}}+\Omega_{\mathrm{b}} \delta_{\mathrm{b}}+\Omega_{\nu} \delta_{\nu}}{\Omega_{\mathrm{cdm}}+\Omega_{\mathrm{b}}+\Omega_{\nu}}\right)^{2}\right\rangle \\
& = \begin{cases}\left\langle\delta_{\mathrm{cdm}}^{2}\right\rangle & \text { for } k<k_{\mathrm{nr}}, \\
{\left[1-\Omega_{\nu} / \Omega_{\mathrm{m}}\right]^{2}\left\langle\delta_{\mathrm{cdm}}^{2}\right\rangle} & \text { for } k \gg k_{\mathrm{nr}},\end{cases}
\end{aligned}
$$

with $\Omega_{\mathrm{m}} \equiv \Omega_{\mathrm{cdm}}+\Omega_{\mathrm{b}}+\Omega_{\nu}$. If the neutrinos did not induce a gravitational backreaction effect on the evolution of the metric and of other perturbations, then $\left\langle\delta_{\text {cdm }}^{2}\right\rangle$ would be the same with and without neutrinos and the role of the neutrino masses would be simply to cut the power spectrum by a factor $\left[1-\Omega_{\nu} / \Omega_{\mathrm{m}}\right]^{2}$ for $k \gg k_{\mathrm{nr}}$. However, we will see in the next subsection that the presence of neutrinos actually modifies the evolution of the CDM and baryon density contrasts in such way that the suppression factor is greatly enhanced, more or less by a factor four.

\subsubsection{Modified evolution of matter perturbations}

\section{Neutrino backreaction effects: generalities.}

In principle, the impact of neutrinos on the evolution of other perturbations (baryons, cold dark matter, photons) can be divided in two categories:

(1) effects from the homogeneous density and pressure of neutrinos, which affect the Friedmann law. Since the equations of evolution of all perturbations contain the expansion rate, the solutions can be affected.

(2) direct gravitational back-reaction effect at the level of perturbations. The neutrino density, pressure and velocity perturbations provide a significant contribution to the total $\delta T_{\mu}^{\nu}$, essentially on scales larger than the free-streaming scale. So, they can change the evolution of metric perturbations, in a similar way as an increased photon density. In addition, 
the neutrino shear induces a non-zero difference $(\phi-\psi)$, which is constant on super-horizon scale during radiation domination and becomes progressively negligible on all scales during matter domination.

Some of these effects are independent of the neutrino mass, and their combination results in a very significant difference between the CMB and LSS power spectra in absence of neutrinos, and those in presence of three families of ultrarelativistic neutrinos (see Fig. 14). These effects are investigated in details in [91], and are extremely relevant from the observational point of view. For instance, they give an opportunity to measure the effective neutrino number $N_{\text {eff }}$ (see Sec. 3.2). Also, it was shown in Ref. [92] that the specific gravitational backreaction effect induced by neutrino shear is actually confirmed by current observations of the CMB temperature spectrum by WMAP.

However, in this review, we are more interested in the effect of the neutrino mass, in realistic scenarios for which the non-relativistic transition of at least two species takes place during matter domination. Our goal is to understand how the matter power spectrum of Eq. (124) depends on the neutrino mass in the two limits $k<k_{\mathrm{nr}}$ and $k>k_{\mathrm{nr}}$. Therefore, we should study how the evolution of $\delta_{\mathrm{cdm}}=\delta_{\mathrm{b}}$ is affected by non-relativistic neutrinos deep in the matter-dominated regime.

\section{How neutrinos slow down the growth of matter perturbations.}

We are arriving to the most important point of this section, since we are going to derive the dominant observable effect of neutrino masses. Before getting into the equations, let us try to describe the effect qualitatively. For this purpose, we first need to come back to the issue of linear clustering of CDM and baryons during matter domination in absence of neutrinos. In Sec. 4.4, we stated that $\phi=\psi$ remains constant during matter domination, basing ourselves on Eq. (58) with a zero sound speed (or equivalently on the perturbed Einstein Eqs. (37) and (38) with zero pressure perturbation and shear on the right-hand side). Then, we used the Poisson equation

$$
-\frac{k^{2}}{a^{2}} \psi=4 \pi G \delta \rho,
$$

to show that inside the Hubble radius, the density contrast $\delta_{\mathrm{cdm}}=\delta_{\mathrm{b}}$ grows like $\left[a^{2}\left(\bar{\rho}_{\mathrm{cdm}}+\bar{\rho}_{\mathrm{b}}\right)\right]^{-1}$, i.e. like a linear function of the scale factor. The fact that linear structure formation corresponds to a constant gravitational potential sounds counter-intuitive at first sight. Actually, it results from an exact compensation between clustering and the stretching of spacetime. During gravitational collapse, the non-relativistic matter density perturbations $\delta \rho$ do not grow with time, as one would naively expect from a common intuition based on a static Universe. Instead, $\delta \rho$ decreases, but only like $a^{-2}$ and not as fast as the dilution factor $a^{-3}$, so that the density contrasts $\delta \rho / \bar{\rho}$ actually grows 
like $a$. The behavior $\delta \rho \propto a^{-2}$ corresponds precisely to a static potential, since the gradient $\Delta \psi=-(k / a)^{2} \psi$ also decreases like $a^{-2}$ due to the stretching of space-time.

Let us restore now the neutrino background. During matter domination and on scales smaller than the free-streaming scale, the neutrino perturbations $\delta_{\nu}$ do not contribute to gravitational clustering. Indeed, we have seen in Secs. 4.5.4 and 4.5.5 that free-streaming leads to $\delta_{\nu} \ll \delta_{\text {cdm }}$, and since $\bar{\rho}_{\nu}<\bar{\rho}_{\text {cdm }}$ we see that $\delta \bar{\rho}_{\nu} \ll \delta \bar{\rho}_{\text {cdm }}$ : so, neutrinos can be simply omitted from the Poisson equation. On the other hand, they do contribute to the homogeneous expansion through Friedmann equation. Therefore the exact compensation between clustering and expansion described in the previous paragraph is slightly shifted: the balance is displaced in favour of the expansion effect, and we expect $\phi=\psi$ to decay slowly, while $\delta_{\text {cdm }}=\delta_{\mathrm{b}}$ should grow not as fast as the scale factor. This mechanism, first described and quantified in Ref. [70], belongs to the first of the two categories defined at the beginning of this subsection: physically, it is a pure background effect, which leads to a modified evolution of metric and matter perturbations.

Let us estimate the magnitude of this effect in the same fashion as in the pioneering paper [70]. At any time we can combine the continuity and Euler equations of CDM perturbations (39) and (40) into

$$
\ddot{\delta}_{\mathrm{cdm}}+\frac{\dot{a}}{a} \dot{\delta}_{\mathrm{cdm}}=-k^{2} \phi+3\left(\ddot{\psi}+\frac{\dot{a}}{a} \dot{\psi}\right)
$$

but well-inside the Hubble radius the source term is dominated by the comoving gradient $-k^{2} \phi$, which is approximately equal to $-k^{2} \psi$ as soon as the neutrino shear can be neglected. This gradient is given by the Poisson equation, and we obtain

$$
\ddot{\delta}_{\mathrm{cdm}}+\frac{\dot{a}}{a} \dot{\delta}_{\mathrm{cdm}}=4 \pi G a^{2} \delta \rho
$$

where $\delta \rho$ is the total density perturbation. In absence of neutrinos and deep inside the matter-dominated regime, we would have $\delta_{\mathrm{cdm}}=\delta_{\mathrm{b}}$, a total density perturbation $\delta \rho=\left(\bar{\rho}_{\mathrm{cdm}}+\bar{\rho}_{\mathrm{b}}\right) \delta_{\mathrm{cdm}}$, an expansion rate given by $3(\dot{a} / a)^{2}=$ $8 \pi G a^{2}\left(\bar{\rho}_{\mathrm{cdm}}+\bar{\rho}_{\mathrm{b}}\right) \propto a^{-1}$, a scale factor $a \propto \tau^{2}$, and the equation would read

$$
\ddot{\delta}_{\mathrm{cdm}}+\frac{2}{\tau} \dot{\delta}_{\mathrm{cdm}}-\frac{6}{\tau^{2}} \delta_{\mathrm{cdm}}=0
$$

with two solutions $\delta_{\text {cdm }} \propto \tau^{2}$ and $\delta_{\text {cdm }} \propto \tau^{-3}$. Neglecting the decaying mode we recover the standard result $\delta_{\mathrm{cdm}} \propto a$. Now, let us consider instead the case with massive neutrinos, still deep inside the matter-dominated regime and 
on scales $k \gg k_{\mathrm{nr}}$, so that $\delta \rho_{\nu}$ does not contribute to the Poisson equation: $\delta \rho=\left(\bar{\rho}_{\mathrm{cdm}}+\bar{\rho}_{\mathrm{b}}\right) \delta_{\mathrm{cdm}}$, while the neutrino background density does contribute to the expansion rate: $3(\dot{a} / a)^{2}=8 \pi G a^{2}\left(\bar{\rho}_{\mathrm{cdm}}+\bar{\rho}_{\mathrm{b}}+\bar{\rho}_{\nu}\right)$. Let us assume that $\bar{\rho}_{\nu}$ is dominated by non-relativistic neutrinos, so that it decays approximately like $a^{-3}$, and the number

$$
f_{\nu} \equiv \frac{\rho_{\nu}}{\left(\rho_{\mathrm{cdm}}+\rho_{\mathrm{b}}+\rho_{\nu}\right)}=\frac{\Omega_{\nu}}{\Omega_{\mathrm{m}}}
$$

remains approximately constant. Then, the scale factor still evolves like $\tau^{2}$ and the equation of evolution reads

$$
\ddot{\delta}_{\mathrm{cdm}}+\frac{2}{\tau} \dot{\delta}_{\mathrm{cdm}}-\frac{6}{\tau^{2}}\left(1-f_{\nu}\right) \delta_{\mathrm{cdm}}=0
$$

Looking for solutions in $\delta_{\mathrm{cdm}} \propto \tau^{2 p}$, we find two roots

$$
p_{ \pm}=\frac{-1 \pm \sqrt{1+24\left(1-f_{\nu}\right)}}{4}
$$

and we conclude that the growing solution for the CDM density contrast reads

$$
\delta_{\mathrm{cdm}} \propto a^{p_{+}} \simeq a^{1-\frac{3}{5} f_{\nu}},
$$

where in the last step we assumed $f_{\nu} \ll 1$. As expected, the growth of $\delta_{\text {cdm }}$ is reduced due to the fact that one of the component in the Universe contributes to the homogeneous expansion rate but not to the gravitational clustering. The Poisson equation gives

$$
-k^{2} \psi \propto a^{p_{+}-1} \simeq a^{-\frac{3}{5} f_{\nu}}
$$

showing that for the same reason the gravitational potential slowly decays during matter domination.

At the end of matter domination and during $\Lambda$ domination, we have already seen that in absence of neutrinos $\phi$ decays like $g(a)$ and $\delta_{\text {cdm }}$ grows like a $g(a)$ (we recall that the damping factor $g(a)$ is normalized to $g=1$ for $a \ll a_{\Lambda}$ ). The combined effect of $\Lambda$ and of neutrinos on the growth of $\delta_{\text {cdm }}$ can be well approximated by [93]

$$
\delta_{\mathrm{cdm}} \propto[a g(a)]^{p_{+}} \simeq[a g(a)]^{1-\frac{3}{5} f_{\nu}} .
$$

\section{Matter power spectrum for massive versus massless neutrinos.}

Let us try to predict analytically the difference between the power spectrum 
of two cosmological models with respectively massive and massless neutrinos $(\Lambda \mathrm{MDM}$ versus $\Lambda \mathrm{CDM})$ assuming that they share the same values of $\left(\omega_{\mathrm{m}}, \omega_{\mathrm{b}}\right.$, $\left.\Omega_{\Lambda}, A, n_{s}, \tau\right)$. The difference between the two models lies in the values of $\omega_{\nu}$ and $\omega_{\mathrm{cdm}}=\omega_{\mathrm{m}}-\omega_{\mathrm{b}}-\omega_{\nu}$, and can be conveniently parametrized by $f_{\nu}$.

(1) for very large scales $k<k_{\mathrm{nr}}$, neutrino perturbations were never affected by free streaming, and in the non-relativistic regime they become indistinguishable from CDM perturbations. In particular, inside the Hubble radius, neutrinos contribute both to the Poisson equation with $\delta_{\nu}=\delta_{\text {cdm }}$ and to the background evolution, so the density contrast grows like $a$. So, for fixed $\omega_{\mathrm{m}}$, this branch of the spectrum is completely insensitive to the value of $f_{\nu}$ and of the neutrino mass.

(2) for very small scales with $k \gg k_{\text {nr }}$ and $k \gg k_{\text {eq }}$, the two spectra will differ for various reasons. First, equality does not take place for the same value of the scale factor in the two models, since one always has $\left(a_{\text {eq }} / a_{0}\right)=$ $\omega_{\mathrm{r}} /\left(\omega_{\mathrm{b}}+\omega_{\mathrm{cdm}}\right)=\left(1-f_{\nu}\right)^{-1} \omega_{\mathrm{r}} / \omega_{\mathrm{m}}$, where $\omega_{\mathrm{r}}$ includes the density of photons and of three effectively massless neutrinos. Since the two models share the same value of $\omega_{\mathrm{r}}$ and $\omega_{\mathrm{m}}$, the ratio between the two values of the scale factor at equality is $a_{\mathrm{eq}}^{f_{\nu}} / a_{\mathrm{eq}}^{f_{\nu}=0}=\left(1-f_{\nu}\right)^{-1}$. At equality, and more generally at any time before the non-relativistic transition, the two models are rigorously equivalent, modulo a shift in the scale factor by the above factor. So, the identity

$$
\delta_{\mathrm{cdm}}^{f_{\nu}}[a]=\delta_{\mathrm{cdm}}^{f_{\nu}=0}\left[\left(1-f_{\nu}\right) a\right]
$$

is valid as long as $a \leq a_{\mathrm{nr}}$. After $a=a_{\mathrm{nr}}$ and in the massive model, the mechanism described in Eqs. (130)-(133) will take place. So, in the approximation of a time-independent fraction $f_{\nu}$ for $a \geq a_{\mathrm{nr}}$ (which is not strictly valid in the case of several massive non-degenerate neutrinos), the growth of CDM perturbations is given by Eq. (134), i.e. by a constant logarithmic slope $\left(1-\frac{3}{5} f_{\nu}\right)$ for the scale factor (or for the product $[a g(a)]$ during dark energy domination),

$$
\delta_{\mathrm{cdm}}^{f_{\nu}}\left[a_{0}\right]=\left(\frac{a_{0} g\left(a_{0}\right)}{a_{\mathrm{nr}}}\right)^{1-\frac{3}{5} f_{\nu}} \delta_{\mathrm{cdm}}^{f_{\nu}}\left[a_{\mathrm{nr}}\right]
$$

In the massless case, everything is identical to the massive model until the time at which $a=\left(1-f_{\nu}\right) a_{\mathrm{nr}}$ (i.e. until the value of the scale factor for which neutrinos become non-relativistic in the massive case "shifted back" to the massless case according to Eq.(135)). Therefore, at this time, the logarithmic slope of $\delta_{\text {cdm }}^{f_{\nu}=0}$ must also be equal to $\left(1-\frac{3}{5} f_{\nu}\right)$, but then it will increase progressively until unity, so that $\delta_{\text {cdm }}^{f_{\nu}=0}$ becomes a linear 
function of $a$ or $[a g(a)]$. In a crude approximation, we can write

$$
\delta_{\mathrm{cdm}}^{f_{\nu}=0}\left[a_{0}\right] \simeq\left(\frac{a_{0} g\left(a_{0}\right)}{\left(1-f_{\nu}\right) a_{\mathrm{nr}}}\right) \delta_{\mathrm{cdm}}^{f_{\nu}=0}\left[\left(1-f_{\nu}\right) a_{\mathrm{nr}}\right]
$$

but this tends to overestimate the growth of perturbations in the massless case: it assumes that right after $a=a_{\mathrm{nr}}$ the logarithmic slope is equal to one, which is not true immediately. Indeed, a comparison with numerical results shows that the total growth factor is a bit smaller,

$$
\delta_{\mathrm{cdm}}^{f_{\nu}=0}\left[a_{0}\right] \simeq\left(\frac{a_{0} g\left(a_{0}\right)}{\left(1-f_{\nu}\right)^{1 / 2} a_{\mathrm{nr}}}\right) \delta_{\mathrm{cdm}}^{f_{\nu}=0}\left[\left(1-f_{\nu}\right) a_{\mathrm{nr}}\right]
$$

Using this semi-analytic result, we find that the ratio between the present value of $\delta_{\mathrm{cdm}}$ in the two models reads

$$
\frac{\delta_{\mathrm{cdm}}^{f_{\nu}}\left[a_{0}\right]}{\delta_{\mathrm{cdm}}^{f_{\nu}=0}\left[a_{0}\right]}=\left(1-f_{\nu}\right)^{1 / 2}\left(\frac{a_{0} g\left(a_{0}\right)}{a_{\mathrm{nr}}}\right)^{-\frac{3}{5} f_{\nu}} .
$$

According to Eq. (124) this means that the total matter power spectrum is reduced by

$$
\frac{P(k)^{f_{\nu}}}{P(k)^{f_{\nu}=0}}=\left(1-f_{\nu}\right)^{3}\left(\frac{a_{0} g\left(a_{0}\right)}{a_{\mathrm{nr}}}\right)^{-\frac{6}{5} f_{\nu}} .
$$

Finally, we can replace $\left(a_{0} / a_{\mathrm{nr}}\right)$ by $2000 m_{\nu} /(1 \mathrm{eV})$ and, assuming that the mass $m_{\nu}$ is shared by a number $N_{\nu}$ of families, we can use $m_{\nu}=$ $\left(\omega_{\nu} / N_{\nu}\right) 93.2 \mathrm{eV}$. We obtain an expression that depends only on $\left(f_{\nu}, N_{\nu}\right.$, $\left.\omega_{\mathrm{m}}, \Omega_{\Lambda}\right)$

$$
\frac{P(k)^{f_{\nu}}}{P(k)^{f_{\nu}=0}}=\left(1-f_{\nu}\right)^{3}\left[1.9 \times 10^{5} g\left(a_{0}\right) \omega_{\mathrm{m}} f_{\nu} / N_{\nu}\right]^{-\frac{6}{5} f_{\nu}}
$$

We show in Fig. 12 that this semi-analytic expression is a very good approximation of the exact numerical result, and also that for plausible values of $\left(\omega_{\mathrm{m}}, N_{\nu}, \Omega_{\Lambda}\right)$ and for $f_{\nu}<0.07$, it can be approximated by the well-known linear expression [94]

$$
\frac{P(k)^{f_{\nu}}}{P(k)^{f_{\nu}=0}} \simeq-8 f_{\nu}
$$

In conclusion -and as confirmed by the numerical simulations in the next subsection - the ratio $P(k)^{f_{\nu}} / P(k)^{f_{\nu}=0}$ computed for fixed parameters $\left(\omega_{\mathrm{m}}\right.$, $\left.\Omega_{\Lambda}\right)$ smoothly interpolates between one for $k<k_{\mathrm{nr}}$ and a plateau given by Eq. (141) for $k \gg k_{\text {eq }}$. 

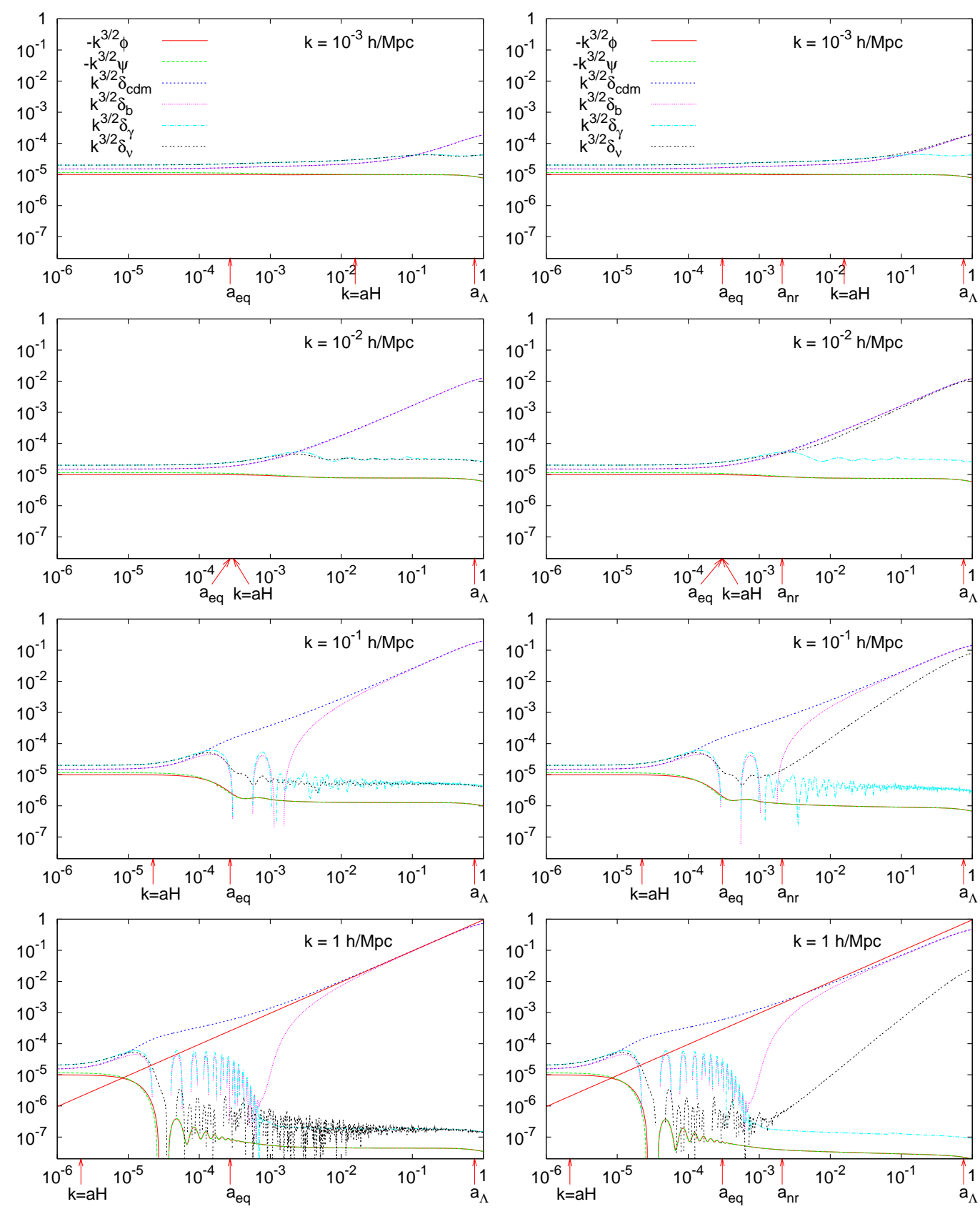

Fig. 11. Evolution of the metric and density perturbations as a function of the scale factor (normalized to $a_{0}=1$ today), in the longitudinal gauge, for modes $10^{-3} h \mathrm{Mpc}^{-1}<k<1 h \mathrm{Mpc}^{-1}$ (from top to bottom), and for two cosmological models: $\Lambda \mathrm{CDM}$ (left) and $\Lambda \mathrm{MDM}$ (right), both with $\omega_{\mathrm{m}}=0.147$ and $\Omega_{\Lambda}=0.7$. The integration has been performed with the code CMBFAST starting from the initial condition $k^{3 / 2} \phi=-10^{-5}$. The $\Lambda \mathrm{MDM}$ model has three degenerate neutrinos with $m_{\nu}=0.46 \mathrm{eV}$, corresponding to $f_{\nu}=0.1$. 


\subsubsection{Numerical results}

In Fig. 11, we tried to summarize all the features described in this section by showing the numerical solutions for the metric and density perturbations $(\phi$, $\left.\psi, \delta_{\mathrm{cdm}}, \delta_{\mathrm{b}}, \delta_{\gamma}, \delta_{\nu}\right)$ at different wavenumbers, for a $\Lambda$ CDM (left) and $\Lambda \mathrm{MDM}$ (right) a model. The two models share common values of $\left(\omega_{\mathrm{m}}, \omega_{b}, \Omega_{\Lambda}, \tau\right)$ and the initial amplitudes are the same. In the $\Lambda$ CDM model, the three neutrino families are massless, while in the $\Lambda \mathrm{MDM}$ model they share a common value of the mass corresponding to $f_{\nu}=0.1$. At the bottom of each plot, we show the value of the scale factor at the time of Hubble entry $(k=a H)$, radiation/matter equality $\left(a_{\mathrm{eq}}\right)$ and matter $/ \Lambda$ equality $\left(a_{\Lambda}\right)$. Decoupling takes place soon after equality, around $a=9 \times 10^{-4}$. These solutions have been computed with the public code CMBFAST [49] in the synchronous gauge, and then translated into the longitudinal gauge. Let us briefly summarize the behavior of each quantity.

For a $\Lambda$ CDM model (left plots in Fig. 11), one has that

- the metric perturbations are essentially constant on large scales $k<k_{\text {eq }}$ during radiation and matter domination. Instead, on small scales $k>k_{\text {eq }}$, they decay between Hubble entry and decoupling, damped by the propagation of acoustic waves. On all scales, they are also damped during $\Lambda$ domination. Initially the difference $\psi-\phi$ is constant and non-zero, due to neutrino shear. After equality, the difference becomes negligible.

- the photon perturbations undergo acoustic oscillations between Hubble entry and decoupling. Then, during matter domination, they free-stream, maintaining and average density contrast $\delta_{\gamma}=-4 \phi$, corresponding (as we have seen) to gravity vs. pressure equilibrium.

- the relativistic neutrino perturbations are damped during radiation domination after Hubble crossing, because of free-streaming. During matter domination free-streaming goes on but $\delta_{\nu}$ maintains itself around $-4 \phi$ like for photons.

- the CDM perturbations always grow inside the Hubble radius. During radiation domination this growth starts abruptly, but then becomes only logarithmic; during matter domination it is linear; finally during $\Lambda$ domination it slows down. In the bottom left plot for $k=1 \mathrm{~h} \mathrm{Mpc}^{-1}$, the solid red line is a linear function of $a$ normalized in order to fit $\delta_{\mathrm{cdm}}$ during matter domination.

- the baryon perturbations follow closely photon perturbations until decoupling, experiencing the same acoustic oscillations. After decoupling, they grow very quickly towards $\delta_{\mathrm{cdm}}$, and after $a \sim 0.01$ the baryon perturbations remain equal to the CDM ones. 


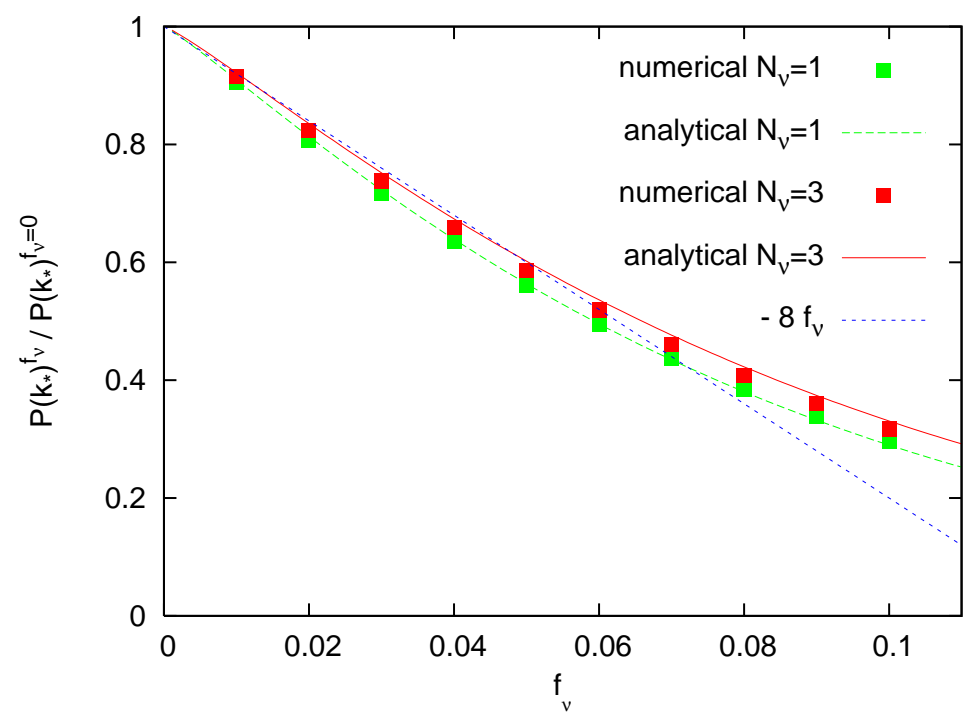

Fig. 12. Ratio of the matter power spectrum including $N_{\nu}=1$ or 3 degenerate massive neutrinos to that with three massless neutrinos, computed at the scale $k_{*}=5 \mathrm{~h} \mathrm{Mpc}^{-1}$ for fixed parameters $\left(\omega_{\mathrm{m}}, \Omega_{\Lambda}\right)=(0.147,0.70)$, and plotted as a function of the density fraction $f_{\nu}$. The numerical result is compared with the semi-analytical approximation of Eq. (141) and with the linear approximation $-8 f_{\nu}$.

For a $\Lambda \mathrm{MDM}$ model (right plots in Fig. 11), the main differences with respect to the previous case are

(1) the fact that equality takes place a bit later, by a fraction $\left(1-f_{\nu}\right)^{-1}=1.1$.

(2) the behavior of $\delta_{\nu}$ after the time of the non-relativistic transition, which is shown at the bottom of the plots by the label " $a_{\mathrm{nr}}$ ". For $a>a_{\mathrm{nr}}$, the neutrino density perturbations track $\delta_{\mathrm{cdm}}=\delta_{\mathrm{b}}$ instead of $\delta_{\gamma}$. For large scales with $k<k_{\text {nr }}$ (upper plot), the non-relativistic transition takes place when the wavelength is larger than the Hubble radius, so $\delta_{\mathrm{cdm}}$ and $\delta_{\nu}$ are not very different when $a=a_{\mathrm{nr}}$, and soon after $\delta_{\nu}$ reaches $\delta_{\mathrm{cdm}}$. For slightly smaller scales like $k=10^{-2} h \mathrm{Mpc}^{-1}$ (right plot in second row), the neutrino perturbations are much smaller than the CDM ones when $a=a_{\mathrm{nr}}$, but then they have time to grow and reach $\delta_{\text {cdm }}$ before the present time. For $k \geq 10^{-1} h \mathrm{Mpc}^{-1}$ (lower plots), they do not have time to do so and remain subdominant today.

(3) the growth of CDM perturbations is suppressed in the $\Lambda$ MDM case for two reasons: first because equality takes place a bit later, and more importantly because $\delta_{\text {cdm }}$ does not grow as fast as the scale factor for $a>a_{\mathrm{nr}}$. This can be seen very clearly on the lower right plot, where we show exactly the same linear function as in the lower left one. The change in the time of equality shifts the $\delta_{\text {cdm }}$ normalization down, while the effect of non-relativistic neutrinos lowers the value of the slope as predicted analytically in the last subsection. 


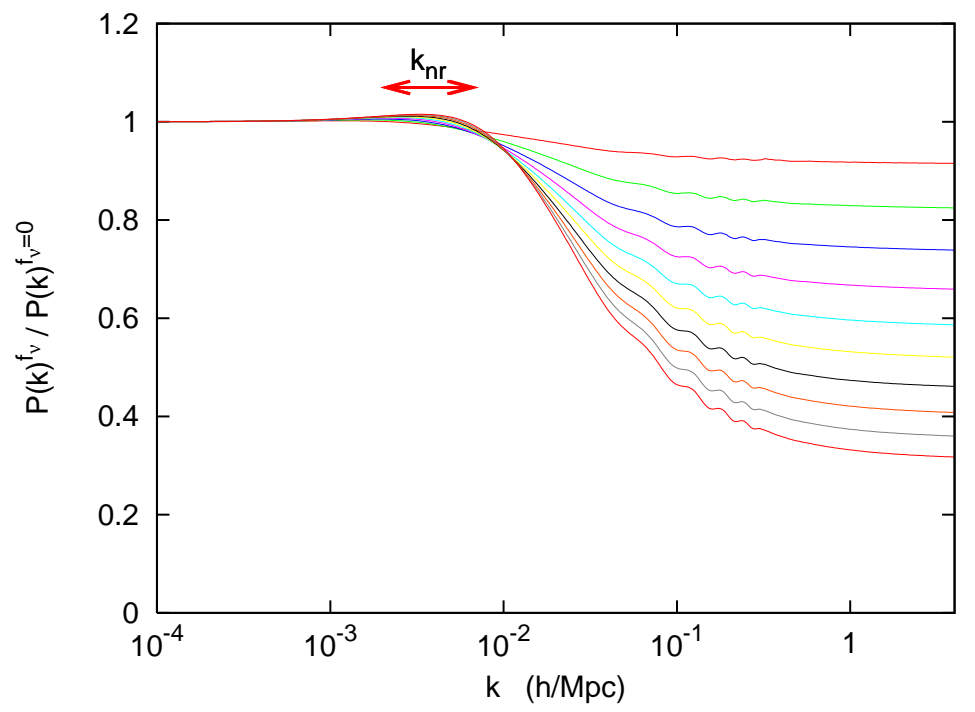

Fig. 13. Ratio of the matter power spectrum including three degenerate massive neutrinos with density fraction $f_{\nu}$ to that with three massless neutrinos. The parameters $\left(\omega_{\mathrm{m}}, \Omega_{\Lambda}\right)=(0.147,0.70)$ are kept fixed, and from top to bottom the curves correspond to $f_{\nu}=0.01,0.02,0.03, \ldots, 0.10$. The individual masses $m_{\nu}$ range from $0.046 \mathrm{eV}$ to $0.46 \mathrm{eV}$, and the scale $k_{\mathrm{nr}}$ from $2.1 \times 10^{-3} h \mathrm{Mpc}^{-1}$ to $6.7 \times 10^{-3} h \mathrm{Mpc}^{-1}$ as shown on the top of the figure. $k_{\text {eq }}$ is approximately equal to $1.5 \times 10^{-2} \mathrm{~h} \mathrm{Mpc}^{-1}$.

Looking now at all wavenumbers, we plot in Fig. 12 the ratio of the matter power spectrum for $\Lambda \mathrm{MDM}$ over that of $\Lambda \mathrm{CDM}$, for different values of $f_{\nu}$, but for fixed parameters $\left(\omega_{\mathrm{m}}, \Omega_{\Lambda}\right)$. Here again, the $\Lambda$ MDM model has three degenerate massive neutrinos. As expected from the analytical results, this ratio is a step-like function, equal to one for $k<k_{\mathrm{nr}}$ and to a constant for $k \gg k_{\text {eq }}$. The value of the small-scale suppression factor is plotted in Fig. 13 as a function of $f_{\nu}$ and of the number $N_{\nu}$ of degenerate massive neutrinos, still for fixed $\left(\omega_{\mathrm{m}}, \Omega_{\Lambda}\right)$. The numerical result is found to be in excellent agreement with the analytical prediction of Eq. (141). For simplicity, the growth factor $g\left(a_{0}\right) \simeq 0.8$ can even be replaced by one in Eq. (141) without changing the result significantly. The well-known formula $P(k)^{f_{\nu}} / P(k)^{f_{\nu}=0} \simeq-8 f_{\nu}$ is a reasonable first-order approximation for $0<f_{\nu}<0.07$.

\subsection{Summary of the neutrino mass effects}

\subsubsection{Effects on $C M B$ and LSS power spectra for fixed $\left(\omega_{\mathrm{m}}, \Omega_{\Lambda}\right)$ and degen- erate masses}

In Fig. 14, we show $C_{l}^{T}$ and $P(k)$ for two models: $\Lambda$ CDM with $f_{\nu}=0$ and $\Lambda \mathrm{MDM}$ with $N_{\nu}=3$ massive neutrinos and a total density fraction $f_{\nu}=0.1$. We also display for comparison the neutrinoless model of Sec. 4.4.6. In all 

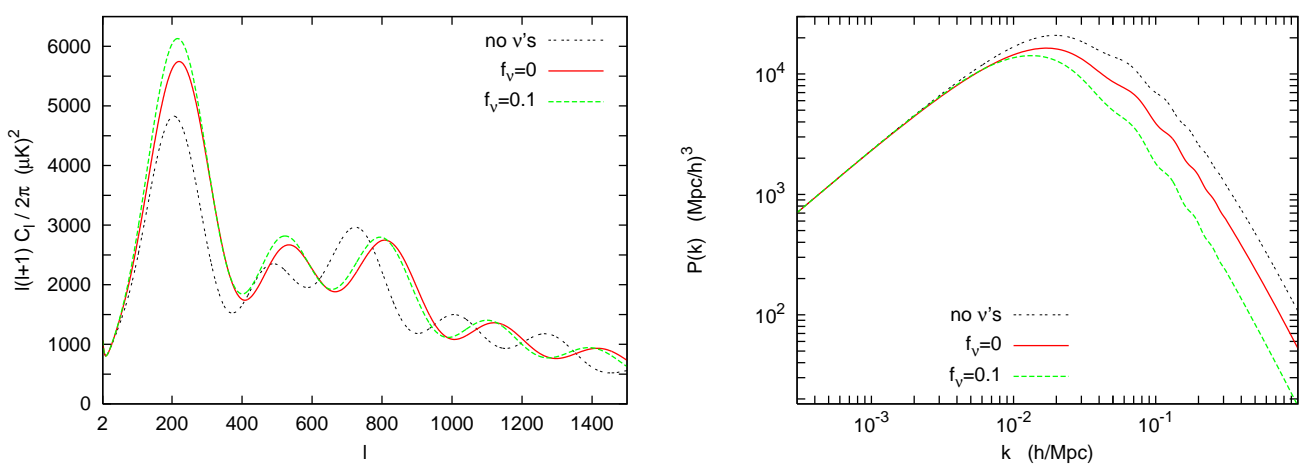

Fig. 14. CMB temperature anisotropy spectrum $C_{l}^{T}$ and matter power spectrum $P(k)$ for three models: the neutrinoless $\Lambda$ CDM model of section 4.4.6, a more realistic $\Lambda$ CDM model with three massless neutrinos $\left(f_{\nu} \simeq 0\right)$, and finally a $\Lambda$ MDM model with three massive degenerate neutrinos and a total density fraction $f_{\nu}=0.1$. In all models, the values of $\left(\omega_{\mathrm{b}}, \omega_{\mathrm{m}}, \Omega_{\Lambda}, A_{s}, n, \tau\right)$ have been kept fixed.
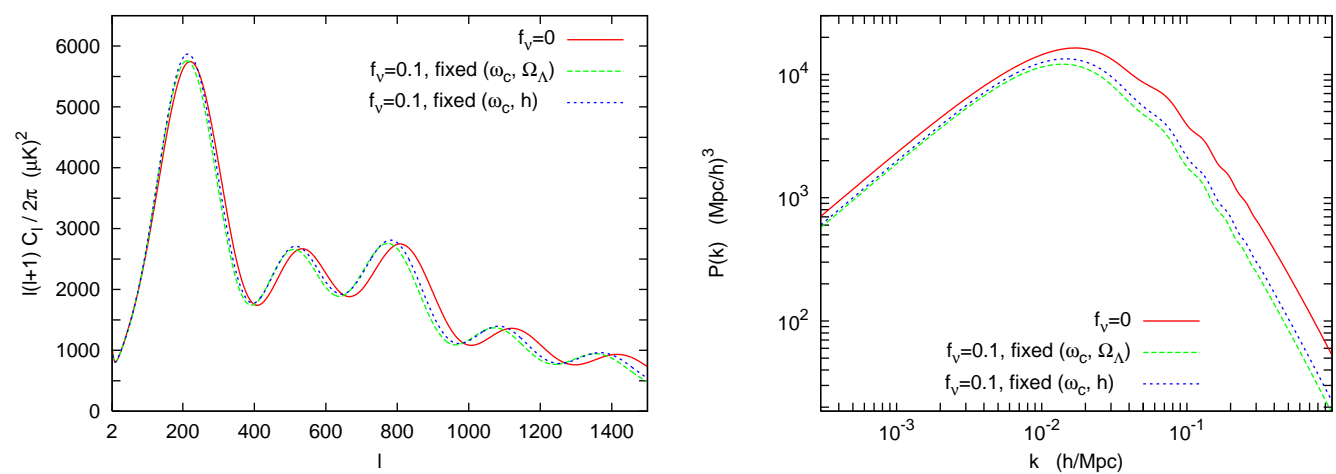

Fig. 15. CMB temperature anisotropy spectrum $C_{l}^{T}$ and matter power spectrum $P(k)$ for three models: the same $\Lambda \mathrm{CDM}$ model as in the previous figure, with three massless neutrinos $\left(f_{\nu} \simeq 0\right)$; and two models with three massive degenerate neutrinos and a total density fraction $f_{\nu}=0.1$, sharing the same value of $\omega_{\mathrm{b}}$ and $\omega_{\mathrm{cdm}}$ as the massless model, which implies a shift either in $h$ (green dashed) or in $\Omega_{\Lambda}$ (blue dotted).

models, the values of $\left(\omega_{\mathrm{b}}, \omega_{\mathrm{m}}, \Omega_{\Lambda}, A_{s}, n, \tau\right)$ have been kept fixed, with the increase in $\omega_{\nu}$ being compensated by a decrease in $\omega_{\mathrm{cdm}}$. There is a clear difference between the neutrinoless and massless neutrino cases, caused by a large change in the time of equality and by the role of the neutrino energymomentum fluctuations in the perturbed Einstein equation [91]. However our purpose is to focus on the impact of the mass, i.e. on the difference between the solid (red) and thick dashed (green) curves in Fig. 14.

Impact on the CMB temperature spectrum. For $f_{\nu} \leq 0.1$, the three neutrino species are still relativistic at the time of decoupling, and the direct effect of free-streaming neutrinos on the evolution of the baryon-photon 
acoustic oscillations is the same in the $\Lambda \mathrm{CDM}$ and $\Lambda \mathrm{MDM}$ cases. Therefore, the effect of the mass is indirect, appearing only at the level of the background evolution: the fact that the neutrinos account today for a fraction $\Omega_{\nu}$ of the critical density implies some change either in the present value of the spatial curvature, or in the relative density of other species. In this section, we choose to maintain a flat Universe with fixed $\left(\omega_{\mathrm{m}}, \omega_{\mathrm{b}}, \Omega_{\Lambda}\right)$-other options would be possible, as discussed in the next section. Thus, while $\Omega_{\mathrm{b}}$ and $\Omega_{\Lambda}$ are constant, $\Omega_{\mathrm{cdm}}$ is constrained to decrease as $\Omega_{\nu}$ increases. The main effect on the CMB anisotropy spectrum results from a change in the time of equality. Since neutrinos are still relativistic at decoupling, they should be counted as radiation instead of matter around the time of equality, which is found by solving $\rho_{\mathrm{b}}+\rho_{\mathrm{cdm}}=\rho_{\gamma}+\rho_{\nu}$. This gives

$$
a_{\mathrm{eq}}=\frac{\omega_{\mathrm{r}}}{\omega_{\mathrm{b}}+\omega_{\mathrm{cdm}}},
$$

where $\omega_{\mathrm{r}}$ stands for the radiation density extrapolated until today assuming that all neutrinos would remain massless, given by Eq. (11) with $N_{\text {eff }}=3.04$. So, when $f_{\nu}$ increases, $a_{\text {eq }}$ increases proportionally to $\left[1-f_{\nu}\right]^{-1}$ : equality is postponed. We already saw in Sec. 4.4.7 that this produces an enhancement of small-scale perturbations, especially near the first acoustic peak. Also, postponing the time of equality increases slightly the size of the sound horizon at recombination. These two features explain why in Fig. 14 the acoustic peaks are slightly enhanced and shifted to the left in the $\Lambda \mathrm{MDM}$ case.

If neutrinos were heavier than a few $\mathrm{eV}$, they would already be non-relativistic at decoupling. This case would have more complicated consequences for the $\mathrm{CMB}$, as described in [85]. However, we will see in section 5 that this situation is disfavoured by current upper bounds on the neutrino mass.

Impact on the matter power spectrum. This issue has been discussed in details in the previous subsection: the combined effect of the shift in the time of equality and of the reduced CDM fluctuation growth during matter domination produces an attenuation of small-scale perturbations for $k>k_{\mathrm{nr}}$, obeying to Eq. (141) in the large $k$ limit. Various studies, including Refs. $[93,95,96,97,98,99]$, have derived some analytical approximation to the full MDM or $\Lambda \mathrm{MDM}$ matter power spectrum, valid for arbitrary scales and redshifts. However, the $\Lambda \neq 0$ case is only considered in Refs. [93,98].

\subsubsection{Degeneracy with other cosmological parameters}

Possible parameter degeneracy in the CMB temperature spectrum. Since the effect of the neutrino mass on CMB fluctuations is indirect and appears only at the background level, one could think that by changing the value 
of other cosmological parameters it would be possible to cancel exactly this effect. For the simplest $\Lambda \mathrm{MDM}$ model with only seven cosmological parameters, this is impossible: one cannot vary the neutrino mass while keeping fixed $a_{\text {eq }}$ and all other quantities governing the CMB spectrum, that we already listed in Sec. 4.4.7. Let us illustrate this with a particular example. Since the time of equality is related to $\left(\omega_{\mathrm{b}}+\omega_{\mathrm{cdm}}\right)$ rather than $\omega_{\mathrm{m}}$, we could try to maintain this sum fixed. In this case, it is now $\omega_{\mathrm{m}}$ that will increase like $\left[1-f_{\nu}\right]^{-1}$. We still have some freedom concerning other parameters: we could choose either to maintain a constant $\Omega_{\Lambda}$, in which case $h$ will increase as $\left[1-f_{\nu}\right]^{-1 / 2}$; or to maintain a constant $h$, in which case $\left(1-\Omega_{\Lambda}\right)$ will increase as $\left[1-f_{\nu}\right]^{-1}$. The result of these two possibilities is displayed on Fig. 15. Since the value of the scale factor at equality is now more or less fixed, the change in peak amplitude is smaller than in Fig. 14. The counterpart is a larger shift in peak location: since we now have variations of either $h$ or $\Omega_{\Lambda}$, the angular scale of the sound horizon at decoupling cannot be kept constant.

We conclude that for a seven parameter $\Lambda \mathrm{MDM}$ model, there is no parameter degeneracy in the CMB spectrum. Therefore, it is possible to constrain the neutrino mass using CMB experiments alone (although neutrinos are still relativistic at decoupling). We will see in the next sections how this applies to current and future experiments.

This conclusion can be altered in more complicated models with extra cosmological parameters. For instance, allowing for an open Universe, it would be possible to increase $f_{\nu}$ with fixed $\left(\omega_{\mathrm{cdm}}, \omega_{\mathrm{b}}, \Omega_{\Lambda}\right)$, and to tune the spatial curvature in order to shift back the peaks to their original location. One could also vary the number of relativistic degrees of freedom in order to play with the time of equality (see e.g. [100]). In such extended models the CMB alone is not sufficient for constraining the mass, but fortunately the LSS power spectrum can lift the degeneracy.

Possible parameter degeneracy in the matter power spectrum. The possibility of a parameter degeneracy in the matter power spectrum depends on the interval $\left[k_{\min }, k_{\max }\right]$ in which it can be accurately measured. Ideally, if we could have $k_{\min } \leq 10^{-2} h \mathrm{Mpc}^{-1}$ and $k_{\max } \geq 1 h \mathrm{Mpc}^{-1}$, the effect of the neutrino mass would be non-degenerate, because of its very characteristic step-like effect. In contrast, parameters like the scalar tilt or the tilt running change the spectrum slope on all scales, while the cosmological parameters which determine the shape of the matter power spectrum below the scale of equality change the slope for all wavenumbers $k \geq k_{\text {eq }}$.

The problem is that usually, the matter power spectrum can only be accurately measured in the intermediate region where the mass effect is neither null nor maximal: in other words, many experiments only have access to the transition region in the step-like transfer function. In this region, the neutrino 
mass affects the slope of the matter power spectrum in a way which can be easily confused with the effect of the scalar tilt, tilt running, or many other cosmological parameters. Because of these parameter degeneracies, the LSS data alone cannot provide significant constraints on the neutrino mass, and it is necessary to combine them with CMB data. The latter usually lift all degeneracies. For instance, the CMB probes primordial fluctuations on a very broad range of scales and makes a very good determination of the tilt and tilt running: so, degeneracies with $n_{s}$ or $d n_{s} / d k$ are usually not a problem. We will see however in Sec. 5.6 that when exotic models with e.g. extra relativistic degrees of freedom, a constant equation-of-state parameter of the dark energy different from -1 or a non-power-law primordial spectrum are introduced, the neutrino mass bound can become significantly weaker.

\subsubsection{Effects of non-degenerate neutrino masses}

It is often said that cosmological perturbations only probe the total neutrino mass $\sum_{i} m_{i}=m_{1}+m_{2}+m_{3}$ (from now on denoted as $M_{\nu}$ ). Let us scrutinize this point.

We saw in the previous subsection that small neutrino masses only have an indirect background effect on the CMB anisotropy spectrum, related to the current density $\omega_{\nu}$, and therefore to the total mass $M_{\nu}$ (see Eq. 12). So, in very good approximation, $\mathrm{CMB}$ anisotropies probe the total mass with no information on the splitting between different mass eigenstates.

As far as LSS is concerned, the situation is a bit more subtle. In Sec. 4.5, we always assumed for simplicity that there was one, two or three massive neutrino species with a common mass. In this case, we have seen that the position of the break in the power spectrum $P(k)$ is related to $k_{\mathrm{nr}}$, i.e. to the time of the non-relativistic transition which depends on the individual mass $m_{\nu}$. Instead, the amplitude of the break is related both to $f_{\nu}$ and $a_{\mathrm{nr}}$, which depend respectively on $M_{\nu}$ and $m_{\nu}$. However, the results in Fig. 12 present a much stronger dependence on $f_{\nu}$ than on $N_{\nu}$ : thus like for the CMB, the dominant effect is that of the total mass, but the individual masses are not completely irrelevant. In conclusion, we expect various scenarios with the same total mass $M_{\nu}$ and different numbers of massive species $N_{\nu}=1,2,3$ to share the same CMB spectrum, but not the same matter power spectrum, with small variations in the scale and amplitude of the damping caused by neutrino free-streaming. For the mass schemes shown in Fig. 1, the normal hierarchy $(\mathrm{NH})$ and the inverted hierarchy $(\mathrm{IH})$, the differences are expected to be even more subtle, because there are three distinct values of $m_{\nu}, a_{\mathrm{nr}}$ and $k_{\mathrm{nr}}$.

This problem has been studied numerically in Ref. [101], using a modified 

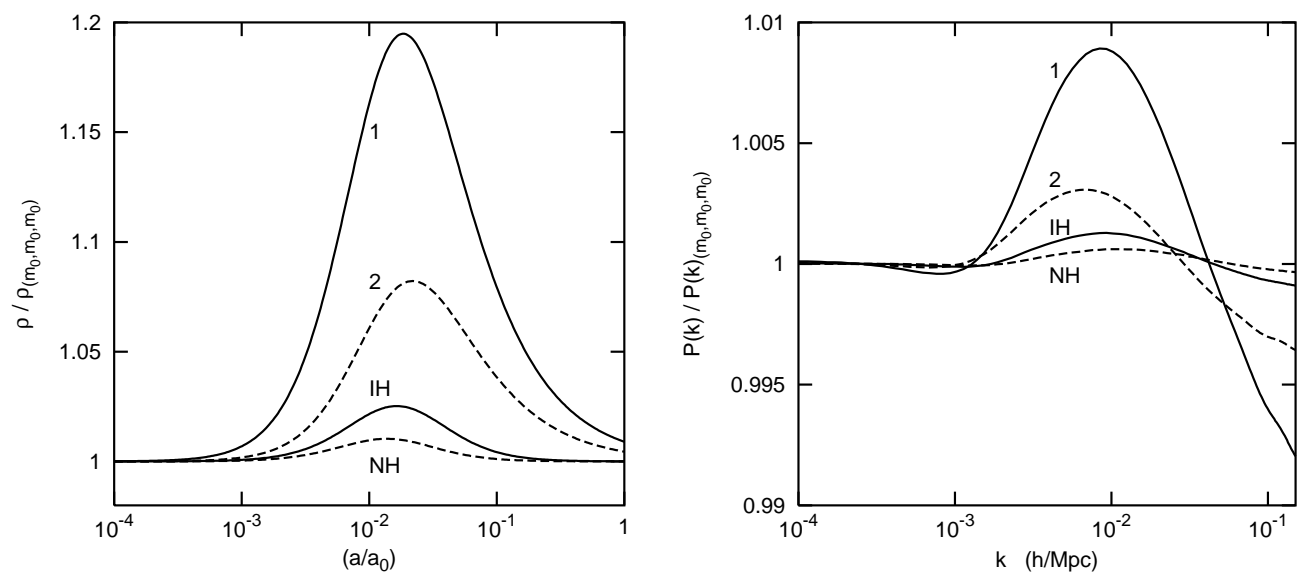

Fig. 16. (Left) Evolution of the total neutrino energy density as a function of the scale factor of the Universe for models where the same total mass $M_{\nu}=0.12 \mathrm{eV}$ is distributed among the three species in various ways. Each line corresponds to the energy density in one of four different cases (only one massive eigenstates (1), two degenerate massive states (2), normal hierarchy (NH), inverted hierarchy (IH)) normalized to the case with three degenerate massive states. (Right) Comparison of the matter power spectrum obtained for the same models, divided each time by that with three degenerate massive states. Differences in the mass splitting affect the position and amplitude of the break in the power spectrum.

version of CMBFAST with three independent neutrino sectors ${ }^{3}$. Fig. 16 is taken from this reference. First, for a fixed total neutrino mass $M_{\nu}=0.12 \mathrm{eV}$, we see on the left plot the ratio of the total neutrino density $\bar{\rho}_{\nu}(a)$ in the $\mathrm{NH}$, IH, $N_{\nu}=1$ or degenerate $N_{\nu}=2$ scenario over that of the degenerate $N_{\nu}=3$ scenario. The bump, more or less pronounced depending on the case, is related to the different epochs of transition to the non-relativistic regime. In the right plot, we can see the ratio between the matter power spectrum $P(k)$ in the same cases over that of the degenerate $N_{\nu}=3$ scenario. The bump around $k=10^{-2} h \mathrm{Mpc}^{-1}$ is caused by the shift in the break scale, and the large- $k$ asymptotic values differ from one because of the different break amplitudes. Generally speaking, the NH scenario interpolates between the $N_{\nu}=1$ and the degenerate $N_{\nu}=3$ cases, depending on the value of the lightest neutrino; the IH case interpolates between the degenerate $N_{\nu}=2$ and $N_{\nu}=3$ cases. But the difference between the $\mathrm{NH}$ and $\mathrm{IH}$ power spectra always remain very small: for instance, in the case shown in Fig. 16 with $M_{\nu}=0.12 \mathrm{eV}$, the two $P(k)$ differ at most by $0.1 \%$. It is very unlikely that this effect can be measured one day (see however section 6).

$\overline{3}$ See also the recent discussion in [102], as well as the study in [103], where CAMB was modified in order to perform calculations with two different neutrino masses. 


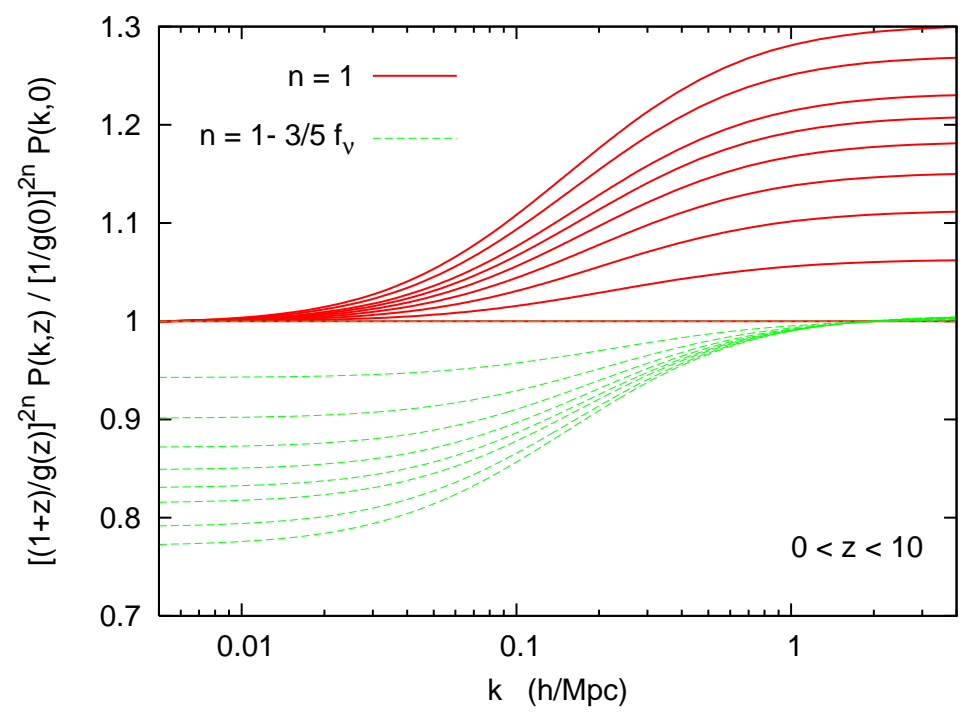

Fig. 17. Shape distortion of the matter power spectrum $P(k, z)$ at different redshifts. Upper solid curves: the power spectra are divided by $[a g(a)]^{2}=[g(z) /(1+z)]^{2}$, which accounts for the evolution on large scales. From top to bottom, the curves correspond to $z=10,8,6,5,4,3,2,1,0$. Lower dashed curves: the power spectra are divided instead by $[a g(a)]^{2-6 / 5 f_{\nu}}=[g(z) /(1+z)]^{2-6 / 5 f_{\nu}}$, which approximates the power spectrum evolution on small scales. From top to bottom, the curves correspond to $z=0,1,2,3,4,5,6,8,10$. All cosmological parameters are kept fixed and the neutrino density fraction is $f_{\nu}=0.1$.

\subsubsection{Massive neutrinos and redshift dependence of the matter power spec- trum}

In absence of neutrinos, we have already seen that the shape of the power spectrum during matter and $\Lambda$ domination is redshift-independent for modes well inside the Hubble radius, while the global power spectrum amplitude grows like $[a g(a)]^{2}$, where $g(a)$ is a damping factor normalized to $g=1$ for $a \ll a_{\Lambda}$ (here $a_{\Lambda}$ is the scale factor at matter $/ \Lambda$ equality). In terms of redshift evolution, this gives

$$
P(k, z)^{f_{\nu}=0}=\left(\frac{g(z)}{(1+z) g(0)}\right)^{2} P(k, 0)^{f_{\nu}=0}, \quad k \gg a H .
$$

The reason for this shape invariance is simply that the matter fluctuations obey to a scale-independent equation of evolution (see for instance Eq. (128), which can easily be generalized to the case of $\Lambda$ domination).

In presence of massive neutrinos, the shape of the power spectrum is not invariant with redshift. Indeed, we are effectively dealing with a two-component system (baryons and CDM on the one hand, neutrinos on the other) in which the largest scales satisfy $\delta_{\nu}=\delta_{\text {cdm }} \propto[a g(a)]$, while for the smallest scales $\delta_{\nu} \ll \delta_{\text {cdm }} \propto[a g(a)]^{1-3 / 5 f_{\nu}}$, as explained in Sec. 4.5.6. Therefore, the power 
spectrum has a different redshift dependence on large and small scales, obeying

$$
P(k, z)=\left\{\begin{array}{rrr}
\left(\frac{g(z)}{(1+z) g(0)}\right)^{2} P(k, 0) & \text { for } \quad a H<k<k_{\mathrm{nr}} \\
\left(\frac{g(z)}{(1+z) g(0)}\right)^{2-6 / 5 f_{\nu}} P(k, 0) & \text { for } & k \gg k_{\mathrm{nr}}
\end{array}\right.
$$

We illustrate this behavior in Fig. 17. Had we plotted the ratio $P(k, z) / P(k, 0)$, we would have seen both the shape and amplitude evolution of the matter power spectrum. Instead, we want to visualize only the shape distortion. So, we divide all power spectra either by $[g(z) /(1+z)]^{2}$ or $[g(z) /(1+z)]^{2-6 / 5 f_{\nu}}$. As expected, in the first case, the largest scales are corrected for their time evolution, and the ratio remains equal to one for $k<0.01 h \mathrm{Mpc}^{-1}$; in the second case, the smallest scales are corrected for their time-evolution and the ratio is equal to one for $k>2 h \mathrm{Mpc}^{-1}$. Between these two limits, the neutrino perturbations are just about to reach the $\delta_{\nu} \longrightarrow \delta_{\text {cdm }}$ asymptotic behavior around the present time (see Sec. 4.5.7). For $f_{\nu}=0.1$, the maximal shape distortion of the matter power spectrum is as large as $6 \%$ for $z=1$ and $15 \%$ for $z=3$.

\section{Current observations and bounds}

In this section we review how the available cosmological data can be used to get information on the absolute scale of neutrino masses, complementary to laboratory experiments. After a brief introduction to the statistical methods used in cosmology (in particular, Bayesian inference), we describe the importance of each set or combination of cosmological data with respect to neutrino masses, and explain how the bounds depend on the data used and the underlying cosmological model. We summarize the current limits in Tables 1-3, where the upper bounds on neutrino masses are given at 95\% C.L. after marginalization over all free cosmological parameters. We briefly discuss how the current bounds on neutrino masses can be relaxed or even avoided when neutrinos possess non-standard properties.

\subsection{Statistical methods for parameter inference from cosmological data}

The theory of cosmological perturbations is a stochastic theory. For a given model, corresponding to some parameter values $\theta_{i}$, the theory predicts the probability of any particular realization, i.e. of any Universe with given per-

turbations. Therefore, it can also predict the probability of obtaining a given 
data set. The likelihood function $\mathcal{L}\left(x_{n} \mid \theta_{i}\right)$ is defined as the probability of observing the data $x_{n}$ given the model parameters $\theta_{i}$. Such a function can be built for any data which has been properly modeled, i.e. for which the instrumental noise properties have been fully understood, and the level of correlation between different data points has been estimated.

The likelihood function can be used as a tool for solving the inverse problem, i.e. measuring the goodness-of-fit of a model given the data, or deriving confidence limits on the model parameters $\theta_{i}$. This highly non-trivial task can be performed in different ways; most particle physicists follow the so-called frequentist approach, while until now, cosmologists usually prefer the Bayesian approach (with very few exceptions, see e.g. $[104,105,106]$ ). None of these methods is better in absolute; they are not directly comparable, because they address different questions. The selection of one method rather than the other should depend of the particular goal of the analysis, on how much the theory is constrained and understood, and on how much we trust the data modeling (for a detailed comparison of the two methods, see [107] or [108] and references therein).

The neutrino mass bounds derived in the next subsections are all based on the Bayesian inference method, which has presumably no secrets for readers with some experience in cosmology. On the other hand, many particle physicists are so familiar with the frequentist philosophy that cosmological bounds leave them perplex. In the rest of this subsection, we briefly summarize the method used for deriving Bayesian confidence limits. Then, we emphasize some properties of Bayesian inference which are radically different from their frequentist counterpart.

In any Bayesian parameter extraction, the first step is to assume a theoretical model, with a set of $N$ free parameters $\theta_{i}$, and $N$ probability distributions $\Pi\left(\theta_{i}\right)$ representing our knowledge on each parameter a priori, before using the data. These priors can be simply flat distributions over the interval in which the parameter is meaningful. For instance, in the case of the neutrino fraction $f_{\nu}$, it seems reasonable to define $\Pi\left(f_{\nu}\right)=1$ for $0<f_{\nu}<1$ and $\Pi\left(f_{\nu}\right)=0$ otherwise: such a prior is called a top-hat. Priors can also contain some information from external data sets. For instance, if one of the free parameters is the current expansion rate $H_{0}$, one can choose to derive its prior from the results of the Hubble Space Telescope (HST) Key Project, and define $\Pi\left(H_{0}\right)$ as a Gaussian probability with central value $72 \mathrm{~km} \mathrm{~s}^{-1} \mathrm{Mpc}^{-1}$ and standard deviation $\sigma=8 \mathrm{~km} \mathrm{~s}^{-1} \mathrm{Mpc}^{-1}$ [109].

Given the likelihood $\mathcal{L}\left(x_{n} \mid \theta_{i}\right)$ and the priors $\Pi\left(\theta_{i}\right)$, one can compute the probability distribution of any parameter $\theta_{j}$, or the joint probability for any subset of parameters. Such a distribution is called a posterior, since it represents the parameter probability a posteriori, after including the full data. Posterior dis- 

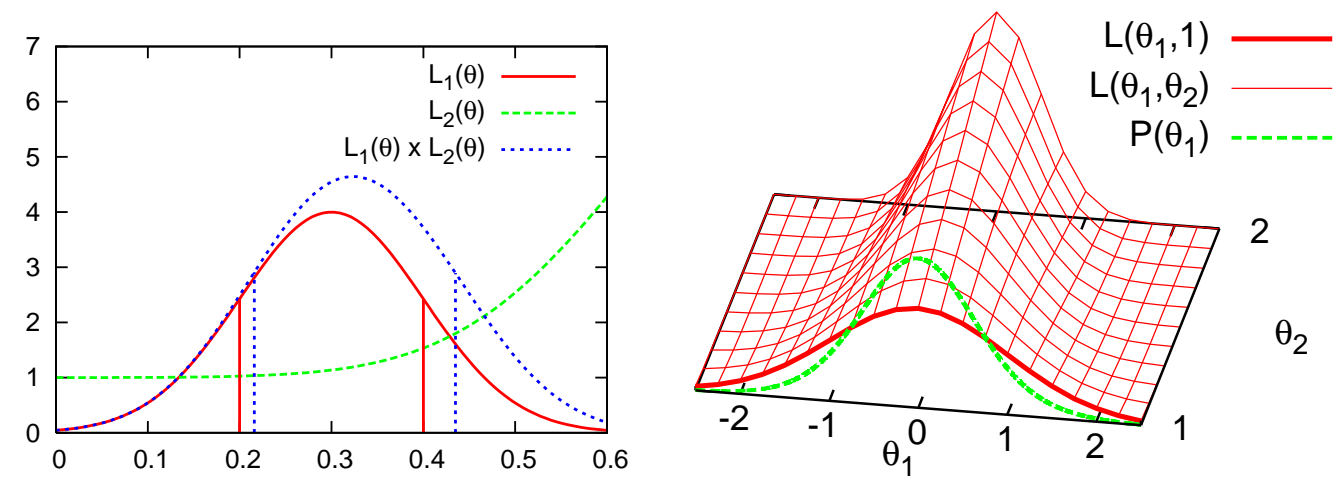

Fig. 18. Situations which are possible in Bayesian inference, while they would be paradoxical in a frequentist analysis. (Left) Adding more data does not always lead to stronger bounds. We consider here a model with one free parameter $\theta$ with flat prior. A first data set has a Gaussian likelihood $L_{1}(\theta)$ which predicts $\theta=0.3 \pm 0.1$ at the $68 \%$ confidence level (this interval corresponds to the solid vertical lines). A second data set has a non-Gaussian likelihood $L_{2}(\theta)$. The product $L_{1}(\theta) \times L_{2}(\theta)$ leads to a wider allowed range, corresponding to the dotted vertical lines. (Right) Adding more free parameters does not always lead to weaker bounds. We study here a two-dimensional model with likelihood $L\left(\theta_{1}, \theta_{2}\right)$ and flat priors. If there are good physical motivations to fix $\theta_{2}=1$, one can also study the one-dimensional model with likelihood $L\left(\theta_{1}, 1\right)$ (the thick line on the plot). We choose the likelihood in such way that $\int d \theta_{1} L\left(\theta_{1}, 1\right)=1$ : the thick line can be directly interpreted as the posterior distribution for $\theta_{1}$ in the one-dimensional model. For the two-dimensional model, the dashed line shows the posterior $P\left(\theta_{1}\right)$ obtained by marginalizing over $\theta_{2}$, and normalizing to one. Given the very specific form of the likelihood, the two-dimensional model leads to a narrower posterior and to stronger bounds on $\theta_{1}$.

tributions are obtained by marginalizing over the remaining free parameters. In practice, this amounts in integrating the product of the likelihood times the priors over these parameters. The result is a function of one or several parameters $\theta_{j}$, which can be normalized to one. One finally obtains a probability distribution $\mathcal{P}\left(\theta_{j}\right)$, from which it is straightforward to derive confidence limits: the $n \%$ confidence interval $I$ is defined as the region in which

(1) the cumulative probability is equal to $n / 100: \int_{I} d \theta_{j} \mathcal{P}\left(\theta_{j}\right)=n / 100$, and

(2) the probability is maximal: the value of $\mathcal{P}\left(\theta_{j}\right)$ for any point inside $I$ is larger than that for any point outside $I$.

The Bayesian inference method is well-defined, efficient, and has several advantages. However, Bayesian results cannot be interpreted in the same way as frequentist ones, and there are significant differences that should be kept in mind:

- Bayesian inference always produce some confidence intervals, even if the 
assumed model is unable to provide good fits to the data. The results of a Bayesian analysis contain no statements on the goodness-of-fit of the model. Therefore, most authors provide some complementary information, like the maximum value of the likelihood $\mathcal{L}_{\max }$ (or equivalently, the minimum value of the effective $\chi^{2}$, which is defined - even for a non-Gaussian likelihood as $\left.\chi_{\text {eff }}^{2}=-2 \ln \mathcal{L}\right)$. Then, it is possible to estimate in the frequentist way how good is the best fit ${ }^{4}$.

- for each parameter, the best-fit value $\theta_{i}^{b}$ generally differs from the most likely value $\theta_{i}^{l}$. Indeed, $\theta_{i}^{b}$ corresponds to the best-fit model, while $\theta_{i}^{l}$ maximizes the marginalization integral. They can be quite different, for instance if for $\theta_{i}$ fixed to $\theta_{i}^{b}$ there are many bad fits and very few excellent fits, while for $\theta_{i}$ fixed to $\theta_{i}^{l}$ there are many good ones. In a future analysis, it would be conceivable that the best-fit value of the total neutrino mass be zero, while the most likely value be strictly positive. This is a natural outcome of the Bayesian approach, which is based on probability distributions in parameter space, and therefore favours regions in which models are good in average.

- the exact meaning of a Bayesian confidence interval is: "given a particular underlying model and given the data, it is likely to be in this region, and unlikely to be outside". One should be careful not to over-interpret a Bayesian confidence interval. It is risky to say that a parameter value sitting outside of the interval is excluded. First, it is possible that with such a value one can find a very good fit to the data (in the frequentist sense): the Bayesian analysis could penalize this point just because the well-fitting region has a tiny volume in the subspace of parameters to be marginalized over. Second, one could possibly assume another model (with extra free parameters) in which the same parameter value would be comfortably allowed. In summary, Bayesian confidence intervals are well-defined, but they are attached to a particular underlying model. It makes no sense to quote a Bayesian upper limit, e.g. on the neutrino masses, without explicitly specifying the model assumed.

- in the frequentist approach, adding more data always lead to stronger bounds on each parameter, while adding more free parameters can only enlarge the allowed region. In the Bayesian approach, this is true in general but not always: it is easy to build counter-examples, as we did in Fig. 18.

Again, Bayesian inference offers many advantages for cosmological parameter extraction, and the previous items should not be regarded as criticisms, but

4 However, comparing the merits of two models is a non-trivial task. A simple comparison of the two maximum likelihood values is insufficient. Following the Bayesian philosophy, the most rigorous approach consists in computing the so-called Bayesian Evidence [110,111]. 
rather as a list of warnings for correctly interpreting the results quoted in this section.

\subsection{CMB anisotropies}

The experimental situation of the measurement of the CMB anisotropies changed radically with the first year release of WMAP data (WMAP1), that includes the temperature $\times$ temperature $(\mathrm{TT})[112]$ and temperature $\times \mathrm{E}$ polarization (TE) [113] correlation functions. The recent third-year release (WMAP3) improves the TT and TE power spectra [114], and adds a detection of the E-polarization self-correlation spectrum (EE) [115]. The WMAP experiment measured with high precision the TT spectrum on large angular scales $(l \lesssim 900)$, while at similar or smaller scales we have results from experiments that are either ground-based such as the Arcminute Cosmology Bolometer Array Receiver (ACBAR) [116], the Very Small Array (VSA) [117], the Cosmic Background Imager (CBI) [118] and the Degree Angular Scale Interferometer (DASI) [119], or balloon-borne such as ARCHEOPS [120], BOOMERANG (TT [121], TE [122] and EE [123]) and MAXIMA [124]. When using data from different $\mathrm{CMB}$ experiments, one should take into account that they overlap in some multipole region, and not all data are uncorrelated.

Small neutrino masses only have a small influence on the power spectrum of CMB anisotropies, since for $m_{\nu}$ smaller than approximately $0.5 \mathrm{eV}$ the neutrinos are still relativistic at the time of recombination, so that their direct effect on the CMB perturbations is identical to that of massless neutrinos. However, the signature of a mass smaller than $0.5 \mathrm{eV}$ does not vanish: we recalled in Sec. 4.6 that there is always a background effect, proportional to $\Omega_{\nu}$, which changes some characteristic times and scales in the evolution of the Universe, and affects mainly the amplitude of the first acoustic peak as well as the location of all the peaks, as shown in Figs. 14 and 15. Therefore, it is possible to constrain neutrino masses using CMB experiments only, down to the level at which this background effect is masked by instrumental noise, or by cosmic variance, or by parameter degeneracies in the case of some cosmological models beyond the minimal $\Lambda$ Mixed Dark matter $(\Lambda \mathrm{MDM})$ framework.

We list in Table 1 the bounds on the sum of neutrino masses that were obtained in analyses of CMB data after the first WMAP results appeared. In these works, a minimal $\Lambda \mathrm{MDM}$ scenario was assumed: the total neutrino mass was the only additional parameter with respect to a flat $\Lambda \mathrm{CDM}$ cosmological model characterized by 6 parameters ${ }^{5}$. However, there were some differences in the analysis procedure.

5 This will be the case for the bounds reviewed in this section, unless specified otherwise. 
Table 1

Upper bounds on $M_{\nu}(\mathrm{eV}, 95 \% \mathrm{CL})$ from recent analyses of CMB data only. Note that the results of Refs. $[127,129,130,131]$ are consistent with each other (see the text for details).

\begin{tabular}{crl}
\hline Ref. & Bound & Data used \\
\hline$[125]$ & 10.6 & WMAP1 \\
{$[127]$} & 2.0 & WMAP1 \\
{$[65]$} & 2.0 & WMAP3 \\
{$[129]$} & 2.1 & WMAP1, VSA, ACBAR \& CBI \\
{$[130]$} & 1.6 & WMAP1, VSA, ACBAR \& CBI \\
{$[131]$} & 3.1 & WMAP1, BOOMERANG03, VSA, ACBAR, \\
& & CBI, DASI \& MAXIMA \\
\hline
\end{tabular}

The analysis in Ref. [125] used a Monte Carlo Markov Chain (MCMC) method for the minimization, concluding that WMAP data are consistent with neutrinos making $100 \%$ of the dark matter component (see also [126]), thus giving the loose bound on the total neutrino mass $M_{\nu}<10.6 \mathrm{eV}$. This would mean that $\mathrm{eV}$ masses are basically indistinguishable from CDM at the epoch of last scattering, and therefore have little impact on the CMB scales measured by WMAP.

However, a very different result was discussed in ref. [127] (see also [128]), where an upper limit of $2 \mathrm{eV}$ was found from the analysis of WMAP1 only using a grid-based method. The authors of this last analysis ascribe the difference with respect to [125] to a better marginalization procedure. However, using the WMAP1 data and an MCMC method, we find the same result as in [127]. Finally, the $2 \mathrm{eV}$ bound is now confirmed by the analysis of the WMAP3 data in Ref. [65].

In Table 1, we also show the results from two recent works [129,131] based on the combination of WMAP1 with other recent CMB experiments. We also show the result of a very recent analysis [130] in a cosmological model with 11 parameters (similar to that in Table 4 of Ref. [132], but in a flat Universe). The $2 \sigma$ bound obtained by $[129,130]$ is still close to $2 \mathrm{eV}$. The result of [131], which include more data from BOOMERANG, DASI and MAXIMA, is larger by $50 \%$. We saw in Sec. 5.1 that in a Bayesian analysis, it is possible to add more data and obtain weaker bounds. In the present case, a careful examination of Fig. 8 in [131] shows that their analysis based on WMAP1 only is in very good agreement with $[127,129]$; however, at least one of the DASI or MAXIMA data set tends to push the bound upwards, because it is better fitted with a non-zero neutrino mass. 
In conclusion, many analyses support the conclusion that a sensible bound on neutrino masses exists, of order of $2-3 \mathrm{eV}$ at $2 \sigma$. This is an important result, since it does not depend on the uncertainties from LSS data (see Sec. 5.3). The only way to relax this bound would be to complicate the cosmological scenario assumed for parameter extraction, but this issue will be discussed in Secs. 5.6 and 5.7 .

However, these results do not take advantage of the most significant observable signature of massive neutrinos: the suppression of small-scale matter fluctuations induced by neutrino free-streaming. In order to get improved bounds on neutrino masses it is crucial to combine CMB data with independent largescale structure observations.

\subsection{Galaxy redshift surveys}

We have seen in Sec. 4.6 that free-streaming of massive neutrinos produces a direct effect on the formation of cosmological structures. As shown in Fig. 13, the presence of neutrino masses leads to an attenuation of the linear matter power spectrum on small scales.

In a seminal paper, $\mathrm{Hu}$ and collaborators [94] showed that an efficient way to probe neutrino masses of order $\mathrm{eV}$ was to use data from large redshift surveys, which measure the distance to a large number of galaxies, giving us a three-dimensional picture of the universe (modulo the effect of unknown peculiar velocities on top of the Hubble flow, which can be corrected only statistically). At present, we have data from two large projects: the 2 degree Field (2dF) galaxy redshift survey [133,134], whose final results were obtained from more than 220,000 galaxy redshifts, and the Sloan Digital Sky Survey (SDSS) [135,89], which will be completed soon with data from one million galaxies ${ }^{6}$.

One of the main goals of galaxy redshift surveys is to reconstruct the power spectrum of matter fluctuations on very large scales, whose cosmological evolution is described entirely by linear perturbation theory. However, the linear power spectrum must be reconstructed from individual galaxies, i.e. from objects which underwent a strongly non-linear evolution. A simple analytic model of structure formation suggests that on large scales, the galaxy-galaxy correlation function should be, not equal, but proportional to the linear matter density power spectrum, up to a constant factor that is called the light-tomass bias. This parameter, denoted by $b$, can be obtained from independent methods (e.g. high-order statistics beyond the two-point correlation function).

${ }_{6}$ An extension of this experiment, SDSS-II was recently funded (see the SDSS webpage http://www.sdss.org) 
Table 2

Upper bounds on $M_{\nu}(\mathrm{eV}, 95 \% \mathrm{CL})$ from recent analyses combining CMB, galaxy redshift surveys (shape of the matter power spectrum only) and other data.

\begin{tabular}{lcl}
\hline Ref. & Bound & Data used (in addition to WMAP1 except for Ref. [65]) \\
\hline$[56]$ & 1.2 & other CMB (pre-WMAP), 2dF-gal \\
& 1.0 & previous + HST, SNIa \\
{$[125]$} & 1.74 & SDSS-gal \\
{$[139]$} & 0.75 & other CMB (pre-WMAP), 2dF-gal, SDSS-gal, HST \\
{$[100]$} & 1.0 & ACBAR, 2dF-gal, SDSS-gal \\
& 0.6 & previous + HST, SNIa \\
{$[132]$} & 0.96 & VSA, 2dF-gal \\
{$[137]$} & 1.54 & SDSS-gal, SNIa \\
{$[140]$} & 1.4 & other CMB , 2dF-gal, HST, SNIa \\
{$[131]$} & 1.2 & other CMB , 2dF-gal, SDSS-gal \\
{$[129]$} & 1.27 & other CMB , SDSS-gal \\
& 1.16 & other CMB , 2dF-gal \\
{$[65]$} & 0.87 & WMAP3, 2dF-gal \\
\hline
\end{tabular}

Currently, these methods tend to confirm that the linear biasing assumption is correct, at least in first approximation.

A conservative way to use the measurements of galaxy-galaxy correlations in an analysis of cosmological data is to take the bias as a free parameter, i.e. to consider only the shape of the matter power spectrum at the corresponding scales and not its amplitude. In this case, we will refer to the corresponding data of the $2 \mathrm{dF}$ and SDSS surveys as $2 \mathrm{dF}$-gal and SDSS-gal, respectively. Alternatively, one can add a measurement of $b$, as recently done in three analyses. The value $b=1.04 \pm 0.11(1 \sigma)$ [2dF-bias] [136] from the $2 \mathrm{dF}$ bispectrum analysis was used in [55], while [137] adopted the estimate $b=0.99 \pm 0.11(1 \sigma)$ [SDSS-bias] from galaxy-galaxy lensing from SDSS [138]. Finally, in Ref. [131] the intermediate estimate $b=1.0 \pm 0.10(1 \sigma)$ was used.

We summarize in Table 2 the bounds on neutrino masses that were obtained in combined analyses of CMB and LSS data, using galaxy clustering data in the range of wavenumbers $(0.015-0.02) h / \mathrm{Mpc}<k<(0.15-0.20) h / \mathrm{Mpc}$ (including non-linear corrections for the smallest wavelengths [141]). The results in this Table were obtained leaving the bias as a free parameter. One concludes from Table 2 that the addition of LSS data improves the bounds with respect to the numbers in Table 1, with an upper limit on $M_{\nu}$ between 
0.9 and $1.7 \mathrm{eV}$ for SDSS-gal and/or 2dF-gal added to CMB data. In general, one obtains weaker bounds on neutrino masses using preliminary SDSS results instead of $2 \mathrm{dF}$ data, but this conclusion is expected to change after the next SDSS releases. The improvement with respect to previous results is evident: in 2001, ref. [142] found $M_{\nu}<4.2 \mathrm{eV}$ (95\% CL) from the combination of the PSCz survey [143] with pre-WMAP CMB data, while the analysis in [144] used early $2 \mathrm{dF}$ data to obtain $M_{\nu}<2.2 \mathrm{eV}$ with priors on the values of $\Omega_{\mathrm{m}}$ and $h$.

There exist other measurements of cosmological parameters that are often added to CMB and LSS data, as shown in Table 2. For instance, the present value of the Hubble parameter was measured by the Key Project of the Hubble Space Telescope (HST), giving $h=0.72 \pm 0.08(1 \sigma)$ [109], which excludes low values of $h$ and leads to a stronger upper bound on the total neutrino mass. In addition, one can include the constraints on the current density of the dark energy component deduced from the redshift dependence of type Ia supernovae (SNIa) luminosity, which measures the late evolution of the expansion rate of the Universe. For a flat Universe with a cosmological constant, these constraints can be translated into bounds for the matter density $\Omega_{\mathrm{m}}$, which range from the conservative value $\Omega_{\mathrm{m}}=0.28 \pm 0.14(1 \sigma)$ from [145] to the more recent $\Omega_{\mathrm{m}}=0.29_{-0.03}^{+0.05}(1 \sigma)$ [146] from the analysis of SNIa with the HST (other recent works include $[147,148]$ ).

As shown in Table 3, the bounds on neutrino masses are more stringent when the amplitude of the matter power spectrum is fixed with a measurement of the bias $[55,131,137]$, instead of leaving it as a free parameter. The upper limits on $M_{\nu}$ are reduced to values of order $0.5-0.9 \mathrm{eV}$ (95\% CL), although some analyses also add Lyman- $\alpha$ data (see next subsection).

Finally, a galaxy redshift survey performed in a large volume can also be sensitive to the imprint created by the baryon acoustic oscillations (BAO) at large scales on the power spectrum of non-relativistic matter, that we discussed in Sec. 4.4 (see Fig. 7). Since baryons are only a subdominant component of the non-relativistic matter, the $\mathrm{BAO}$ feature is manifested as a small single peak in the galaxy correlation function in real space that was recently detected from the analysis of the SDSS luminous red galaxy (LRG) sample [89] at a separation of $100 h^{-1}$ Mpc. The observed position of this baryon oscillation peak provides a way to measure the angular diameter distance out to the typical LRG redshift of $z=0.35$, which in turn can be used to constrain the parameters of the underlying cosmological model. The SDSS measurement of the angular diameter distance at $z=0.35$ [SDSS-BAO] was included in the analysis of Ref. [152] to get a bound of $0.44 \mathrm{eV}$ (95\% CL) on the total neutrino mass $M_{\nu}$, as shown in Table 3. 
Table 3

Upper bounds on $M_{\nu}(\mathrm{eV}, 95 \% \mathrm{CL})$ from recent analyses combining CMB, LSS (including bias and/or $\operatorname{Ly} \alpha$ ) and other cosmological data.

\begin{tabular}{lcl}
\hline Ref. & Bound & Data used (in addition to WMAP1 except for Ref. [65]) \\
\hline$[55]$ & 0.63 & ACBAR, CBI, 2dF-gal, 2dF-bias \\
& 0.68 & previous + Ly $\alpha[149,150]$ \\
{$[137]$} & 0.54 & SDSS-gal, SNIa, SDSS-Ly $\alpha$ \\
& 0.42 & previous + SDSS-bias \\
{$[140]$} & 0.47 & other CMB, 2dF-gal, HST, SNIa, SDSS-Ly $\alpha$ \\
{$[151]$} & 0.65 & SDSS-gal, HST, Ly $\alpha$ [149,150] \\
{$[131]$} & 0.48 & other CMB, 2dF-gal, SDSS-gal, bias $b=1.0 \pm 0.10$ \\
{$[152]$} & 0.44 & other CMB, 2dF-gal, SDSS-gal, HST, SNIa, SDSS-BAO \\
& 0.30 & previous + SDSS-Ly $\alpha$ \\
{$[65]$} & 0.91 & WMAP3, SDSS-gal, SDSS-bias \\
& 0.68 & previous + other CMB, 2dF-gal, SNIa \\
\hline
\end{tabular}

\subsection{Lyman- $\alpha$ forest}

The matter power spectrum on small scales can also be inferred from data on the so-called Lyman- $\alpha$ forest. This corresponds to the Lyman- $\alpha$ absorption of photons traveling from distant quasars $(z \sim 2-3)$ by the neutral hydrogen in the intergalactic medium. As an effect of the Universe expansion, photons are continuously red-shifted along the line of sight, and can be absorbed when they reach a wavelength of $1216 \AA$ in the rest-frame of the intervening medium. Therefore, the quasar spectrum contains a series of absorption lines, whose amplitude as a function of wavelength traces back the density and temperature fluctuations of neutral hydrogen along the line of sight. It is then possible to infer the matter density fluctuations in the linear or quasi-linear regime (see e.g. $[153,154,155,156])$.

In order to use the Lyman- $\alpha$ forest data, one needs to recover the matter power spectrum from the spectrum of the transmitted flux, a task that requires the use of hydro-dynamical simulations for the corresponding cosmological model. This matter power spectrum is again sensitive to the suppression of growth of mass fluctuations caused by massive neutrinos. In 1999, Ref. [157] obtained an upper bound of $m_{\nu}<5.5 \mathrm{eV}$ (for one massive neutrino species) from the combined analysis of Lyman- $\alpha$ data and other cosmological measurements.

Given the various systematics involved in the analysis pipeline, the robustness 
of Lyman- $\alpha$ forest data is still a subject of intense discussion between experts. Among these systematics, we can cite the uncertainty existing on: the mean absorption by the intergalactic medium (the effective optical depth), the temperature-density relation within the absorbing gas, the effect of galactic winds, or the proper use and accuracy of hydro-dynamical simulations in the mildly non-linear regime. Different authors tend to describe or to deal with these uncertainties in slightly different ways $[149,150,158,159]$, an indication that the field might not be fully mature at the moment. In addition, the way in which neutrinos are implemented in hydro-dynamical simulations is generally rather simplistic.

Some of the results shown in Table 3 were obtained adding Lyman- $\alpha$ data to CMB and other LSS data. For a free bias, one finds that Lyman- $\alpha$ data help to reduce the upper bounds on the total neutrino mass: using the SDSS Lyman- $\alpha$ forest [159,160], Ref. [137] finds $M_{\nu}<0.54 \mathrm{eV}$ (95\% CL), while Ref. [151] quotes $0.65 \mathrm{eV}$ using Lyman- $\alpha$ data from $[149,150]$. At least two recent analyses combined results on the bias and the Lyman- $\alpha$ forest: in Ref. [137] the addition of SDSS-bias allowed to reduced the upper bound on $M_{\nu}$ to $0.42 \mathrm{eV}$, while the first year analysis of WMAP collaboration [55] found a slightly weaker bound of $0.68 \mathrm{eV}$ when adding $2 \mathrm{dF}$-bias and Lyman- $\alpha$ data from $[149,150]$. Finally, Ref. [152] found the upper bound $M_{\nu}<0.30 \mathrm{eV}(95 \%$ $\mathrm{CL}$ ) adding simultaneously SDSS-Ly $\alpha$ and SDSS-BAO data.

Thus, at present, the impact of Lyman- $\alpha$ data on the neutrino mass bounds is comparable to that of galaxy bias measurements, if not weaker. We can conclude that both the Lyman- $\alpha$ data and the light-to-mass bias determination are powerful tools for getting information on neutrino masses, but due to the many involved systematics, the current bounds obtained with them are probably not as robust as those in Tables 1 and 2 .

\subsection{Summary of current bounds}

The results shown in Tables 1-3 are representative of an important fact: a single cosmological bound on neutrino masses does not exist. A graphical summary is presented in Fig. 19, where the cosmological bounds correspond to the vertical bands, which were grouped according to the ranges quoted in Tables 1, 2 and 3, respectively. The thickness of these bands roughly describe the spread of values obtained from similar cosmological data: $2-3 \mathrm{eV}$ for CMB only, $0.9-1.7 \mathrm{eV}$ for CMB and $2 \mathrm{dF} / \mathrm{SDSS}$-gal or $0.3-0.9 \mathrm{eV}$ with the inclusion of a measurement of the bias and/or Lyman- $\alpha$ forest data and/or the SDSS measurement of the baryon oscillation peak.

One can see from Fig. 19 that current cosmological data probe the region of 


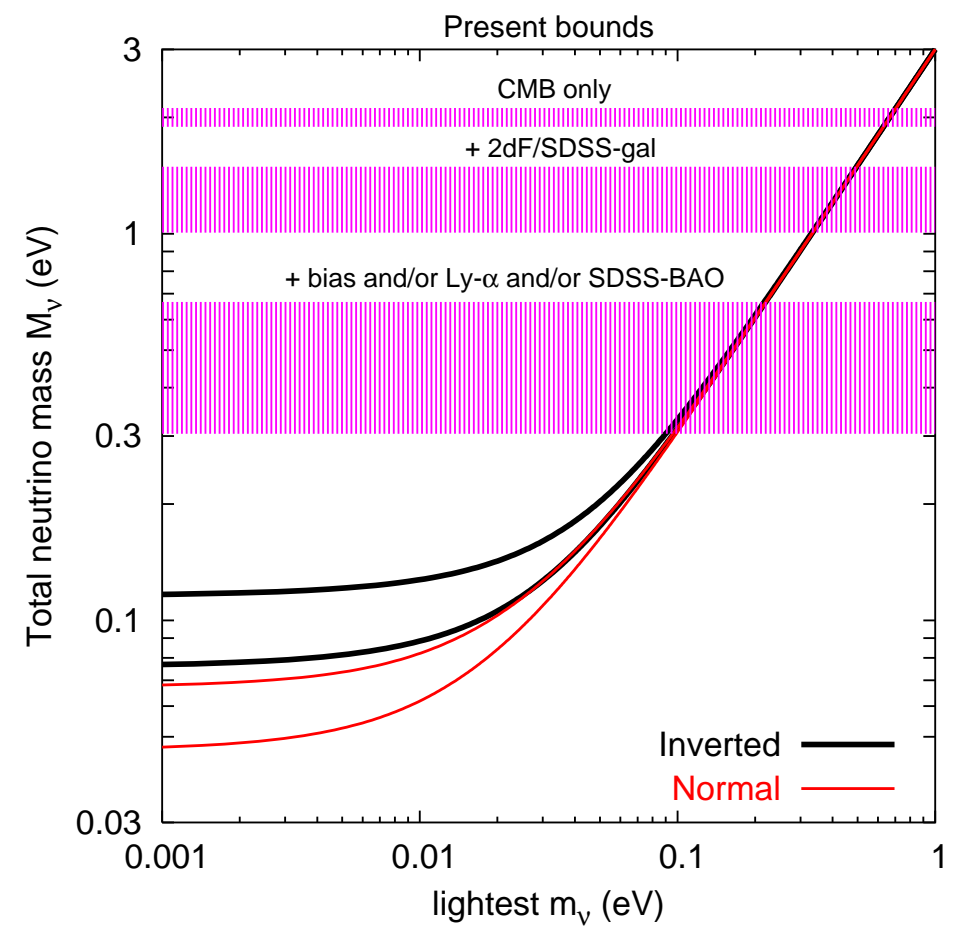

Fig. 19. Current upper bounds (95\%CL) from cosmological data on the sum of neutrino masses, compared to the values in agreement at a $3 \sigma$ level with neutrino oscillation data in Eq. (2).

neutrino masses where the 3 neutrino states are degenerate, with a mass $M_{\nu} / 3$. This mass region is conservatively bounded to values below approximately $1 \mathrm{eV}$ from CMB results combined only with galaxy clustering data from $2 \mathrm{dF}$ and/or SDSS (i.e. the shape of the matter power spectrum for the relevant scales). The addition of further data leads to an improvement of the bounds, which reach the lowest values when data from Lyman- $\alpha$ and/or the SDSS measurement of the baryon oscillation peak are included or the bias is fixed. In such cases the contribution of a total neutrino mass of the order $0.3-0.6 \mathrm{eV}$ seems already disfavoured, in particular covering the recent positive $0 \nu 2 \beta$ signal from [31] (of course with the corresponding caveats, see e.g. the discussion in [14]).

There exist other measurements of cosmological parameters that were not included in the analyses we have reviewed so far, although they could play a role for neutrino mass determination. In particular, we have seen that any measurement that fixes the amplitude of the matter power spectrum, such as the bias, would be very important. For instance, the parameter $\sigma_{8}$-which gives the linear rms mass fluctuations in spheres of radius $8 h^{-1} \mathrm{Mpc}-$ can be measured, for instance, from the determination of cluster masses through Xray observations (see e.g. [161]) or from weak lensing (see e.g. [162]). If various methods happened to converge towards an accurate determination of $\sigma_{8}$, the determination of neutrino masses would improve significantly, as discussed in Ref. [163]. Since $\sigma_{8}$ probes the amplitude of matter fluctuations on scales of 
the order a few Mpc which are deeply affected by neutrino free-streaming, a large value of $\sigma_{8}$ would push down the upper bound on $M_{\nu}$, while a low value would potentially bring evidence for non-zero masses. However, at the moment, the ensemble of $\sigma_{8}$ determinations is rather spread (see Table 5 in [125]), suggesting that not all systematics errors are properly modelled.

One of the first analysis of neutrino mass bounds from cosmology used data on $\sigma_{8}$ from cluster abundance to obtain an upper bound of order a few eV [164]. Another possibility was recently explored in [165], using data on the redshift-evolution of the number density of massive galaxy clusters to find the bound $M_{\nu}<2.4 \mathrm{eV}$ (95\% C.L.). Ref. [166] used another probe of the smallscale power spectrum amplitude from X-ray cluster data, and found some marginal evidence for a non-zero neutrino total mass, $M_{\nu}=0.56_{-0.26}^{+0.30} \mathrm{eV}$ at $68 \%$ C.L. This result is related to the fact that the value of $\sigma_{8}$ preferred by these X-ray data is as small as to contradict most other determinations of this parameter, for instance from weak lensing. This example shows that the sensitivity of cosmological observations to neutrino masses is a powerful tool, but its implications should not be extracted without care.

\subsection{Extra parameters}

Assuming that the relic neutrinos are standard, we have seen that the limits mainly depend on the cosmological data used in the analysis, although minor differences on the quoted bounds exist even when using similar data. These differences arise because not all the analyses in the Tables used the same set of cosmological parameters and priors (such as the assumption of a flat Universe). It is thus important to test whether the impressive cosmological bounds on neutrino masses change much if additional cosmological parameters, beyond those included in the minimal $\Lambda \mathrm{CDM}$, are allowed. This could be the case whenever a new parameter degeneracy with the neutrino masses arises.

An interesting case of degeneracy between cosmological parameters is that between neutrino masses and the radiation content of the universe (parametrized via the effective number of neutrinos $N_{\text {eff }}$ ). The extra radiation partially compensates the effect of neutrino masses, provided that other cosmological parameters such as $\Omega_{\mathrm{m}}$ and $h$ are varied, leading to a less stringent bound on $M_{\nu}[56,100,167,168]$. This parameter degeneracy is shown in Fig. 20, taken from Ref. [100]. For instance, when one considers a model with four instead of three species of massive neutrinos, the upper bound found from CMB and $2 \mathrm{dF} / \mathrm{SDSS}$-gal is relaxed from 0.8 to $1.2 \mathrm{eV}$ when one of the states is much heavier than the others. These results are interesting for the 4-neutrino mass schemes that also incorporate the results of the LSND experiment [19], that we discussed in Sec. 2. At present, the LSND regions in the space of oscillation 

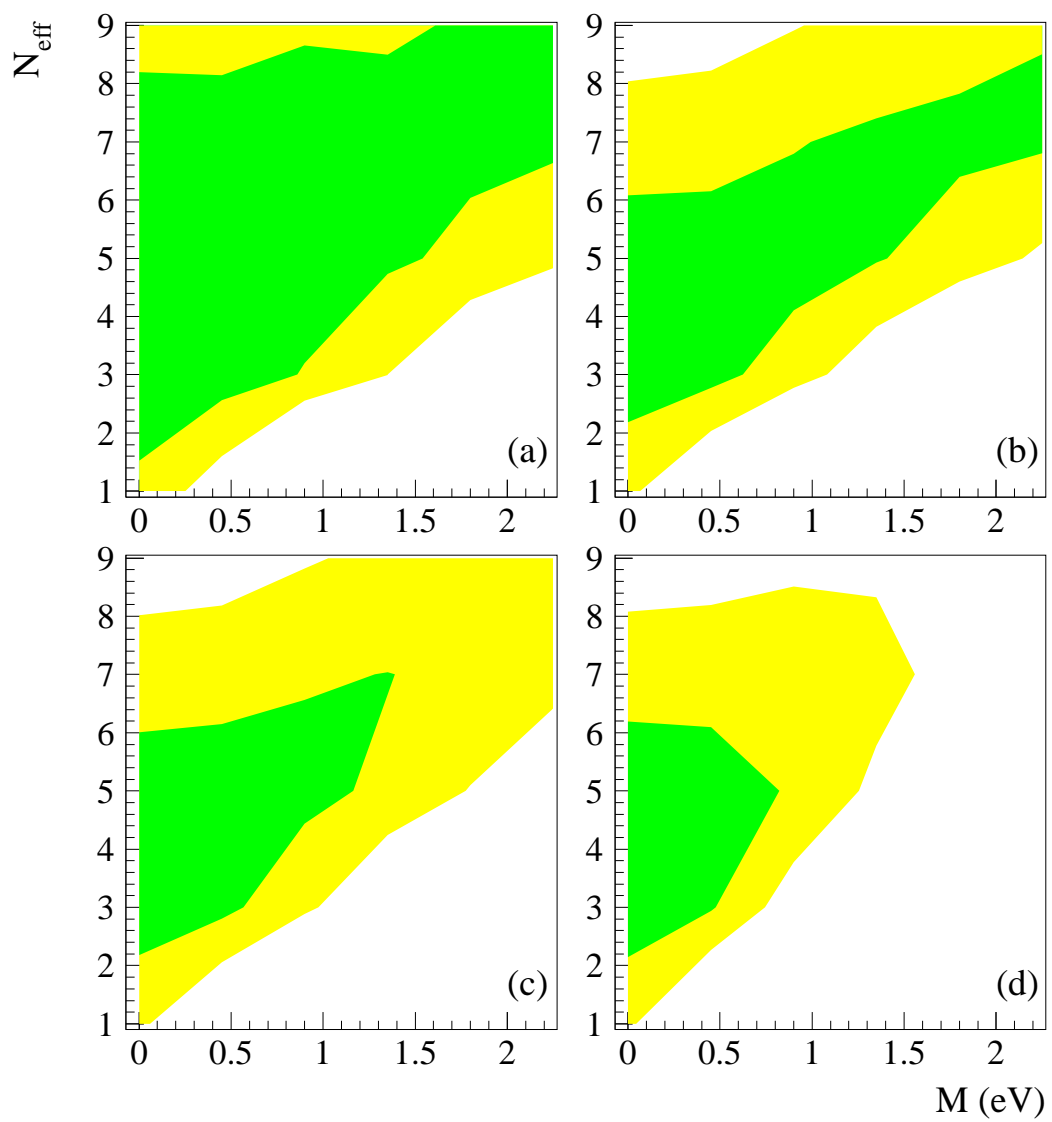

Fig. 20. Two-dimensional likelihood in $\left(N_{\text {eff }}, M_{\nu}\right)$ space, marginalized over the other cosmological parameters of the model. We plot the $1 \sigma$ (green / dark) and $2 \sigma$ (yellow / light) allowed regions. Here we used CMB (WMAP1 \& ACBAR) and LSS (2dF \& SDSS) data, adding extra priors on $h$ (HST) and $\Omega_{\mathrm{m}}$ (SN99 or SN03) as follows: (a) no priors, (b) HST, (c) HST+SN99, (d) HST+SN03. For details, see ref. [100].

parameters are not yet completely disfavoured ${ }^{7}$ by cosmological data.

Recently a degeneracy between neutrino masses and the parameter $w$, that characterizes the equation of state of the dark energy component $X\left(p_{X}=\right.$ $\left.w \rho_{X}\right)$, was studied in [170]. For constant values $w<-1$, in the so-called phantom energy regime (which could describe particular scenarios based on string theory or modified gravity), it was shown that the neutrino mass bound can be relaxed to $M_{\nu}<1.48 \mathrm{eV}$ (95\% C.L.), compared with $M_{\nu}<0.65 \mathrm{eV}$ for fixed $w=-1$. Similar conclusions were obtained by the WMAP team (see Fig. 18 in [65]). Ref. [170] studied the effect of this parameter degeneracy on the analysis of future cosmological data (see also [86] for models with a timevarying equation of state of dark energy). Finally, Ref. [152] showed that data

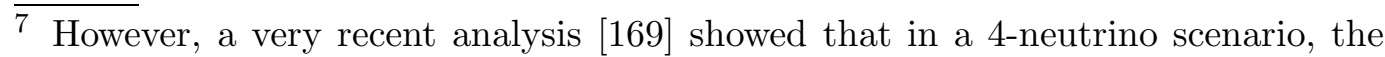
heavier neutrino mass is bounded to values smaller than $0.55 \mathrm{eV}$ (95\% CL) when data from CMB, LSS and Lyman- $\alpha$ are included. 
on baryon acoustic oscillations (see Sec. 5.3) helps in breaking the $m_{\nu}-w$ degeneracy.

Finally, the neutrino mass bounds could certainly be relaxed by assuming that the primordial power spectrum has a non-trivial shape or Broken Scale Invariance (BSI). This eventuality cannot be excluded, for instance if a phase transition takes place during inflation. Such an assumption opens so many degrees of freedom in the parametrization of cosmological models that a systematic study is almost impossible. However, some authors [171] used this possibility in combination with a non-zero neutrino mass for building a cosmological scenario without any form of dark energy, that could fit most of the data available at the time of publication, except for SNIa data. This model is now ruled out by the combination of WMAP with the SDSS galaxy-galaxy correlation function, but it illustrates the fact that if one is ready to add more degrees of freedom in cosmological scenarios (in the case of Ref. [171], they were actually not so many), then there is most probably a way to evade the bounds presented in this review.

\subsection{Non-standard relic neutrinos}

Finally, let us remind the reader that the cosmological implications of neutrino masses could be very different if the spectrum or evolution of the cosmic neutrino background was non-standard. Our aim here is not to give an exhaustive list of all possibilities (many results on non-standard neutrinos were reviewed in [6]), but only to briefly summarize some recent works.

For instance, the bounds on neutrino masses would be modified if relic neutrinos presented a momentum spectrum significantly different from the equilibrium Fermi-Dirac distribution of Eq. (6). For instance, neutrinos could violate the Pauli exclusion principle and obey Bose-Einstein statistics with important cosmological and astrophysical implications [172,173]. Non-equilibrium neutrino spectra can also be produced in low-reheating scenarios $[174,175,176,177,178,179]$, from the decay of massive neutrinos into relativistic products (see e.g. $[180,181]$ ) or as the result of active-sterile neutrino oscillations after decoupling (see $[182,183]$ and references therein). A recent analysis of cosmological data including large non-thermal corrections in the neutrino spectra was done in Ref. [184].

We conclude giving two examples of models where the cosmological bounds on neutrino masses that we have discussed can be evaded. In the first case, massive neutrinos are strongly coupled to a light scalar field, in such a way that they annihilate as soon as they become non-relativistic and do not affect the formation of LSS [185]. The viability of this kind of models depends on 
the cosmological data included in the analysis and on the number of neutrino species strongly interacting (see the discussions in Refs. $[186,187,188]$ ).

The second possibility, described in $[189,190]$ and many other recent works (see e.g. the discussion in [191]), concerns neutrinos coupled to the dark energy so that the dark energy density is a function of the neutrino mass (Mass-Varying Neutrinos or MaVaNs). Then, neutrino masses are only relevant for the very recent stages of the Universe, so that no bounds can be placed from highredshift cosmological data. Note, however, that this is not true for all MaVaNs scenarios, see for instance [192].

\section{Future sensitivities and new experimental techniques}

If the characteristics of future experiments are known with some precision, it is possible to assume a "fiducial model", i.e. a cosmological model that would yield the best fit to future data, and to estimate the error bar on a particular parameter that will be obtained after marginalizing the hypothetical likelihood distribution over all the other free parameters (see Sec. 5.1). Technically, the simplest way to forecast this error is to compute a Fisher matrix, as explained in the next subsection. This technique has been widely used in the literature, for many different models and hypothetical datasets. Here we will focus on the results for $\sigma\left(M_{\nu}\right)$, the forecast $68 \%$ CL error on the total neutrino mass, assuming various combinations of future observations (CMB anisotropies, galaxy redshift surveys, weak lensing surveys, ...) and various cosmological models with fiducial parameter values. Many papers derived some predictions for $\sigma\left(M_{\nu}\right)$; we will summarize here the main results and see whether the expected sensitivities will be sufficient for detecting the total neutrino mass, even if it is close to the minimum values guaranteed by present data on flavour neutrino oscillations.

\subsection{Fisher matrix}

In a Bayesian analysis, the probability distribution in the space of cosmological parameters is inferred from the likelihood $\mathcal{L}\left(x_{n} \mid \theta_{i}\right)$ of the data $x_{n}$ given a theoretical model described by a set of parameters $\theta_{i}$ (see Sec. 5.1). If we assume that the maximum likelihood $\mathcal{L}_{\max }$ is obtained for a particular model $\theta_{i}^{0}$ (the "fiducial model"), and if we approximate the likelihood function as a multivariate Gaussian in the vicinity of the maximum, the symmetrical error 
on each parameter can be inferred from the Fisher matrix, defined as

$$
F_{i j} \equiv-\left.\frac{\partial^{2} \ln \mathcal{L}}{\partial \theta_{i} \partial \theta_{j}}\right|_{\theta_{i}^{0}}
$$

The $1 \sigma$ (or $68 \%$ confidence) error bar on a particular parameter $\theta_{j}$ assuming that all other parameters are fixed to the fiducial values $\theta_{i}^{0}$ would be given by $\left(F_{j j}\right)^{-1 / 2}$. However, the really interesting quantity is the error on $\theta_{j}$ assuming that the other parameters are unknown (and simply marginalized out by integrating over the likelihood). In that case, the error can be shown to be $[193]$

$$
\sigma\left(\theta_{j}\right)=\left(F^{-1}\right)_{j j}^{1 / 2}
$$

Geometrically, and in the multivariate Gaussian approximation, the Fisher matrix gives the coefficients of the ellipsoids $F_{i j} \theta_{i} \theta_{j}=f(c)$ corresponding to given confidence levels $c$ in parameter space. Therefore, the study of its eigenvectors and eigenvalues gives an idea of the linear parameter combinations with the best and poorest determination (the latter correspond to parameter degeneracies).

In many cases one refers to data points $x_{i}(i=1, \ldots, n)$ with a Gaussian probability distribution centered in zero

$$
\mathcal{L}(\vec{x} \mid \vec{\theta})=\left[(2 \pi)^{n}|C|\right]^{-1 / 2} e^{-\frac{1}{2} \vec{x}^{t} C \vec{x}}
$$

where the "data covariance matrix" $C$ contains the variance expected for the data, $C \equiv\left\langle\vec{x} \vec{x}^{t}\right\rangle$, after a detailed modelling of all possible contributions. Typically, the data covariance matrix will be the sum of the theoretical model prediction (for parameters $\vec{\theta}$ ) and of the noise variance estimated from the instrumental characteristics. In general, the data covariance matrix is nondiagonal and the data points are correlated with each other. A purely algebraic calculation shows that the Gaussian likelihood of Eq. (148) inserted in Eq. (146) leads to the following expression for the Fisher matrix [193]

$$
F_{i j}=\frac{1}{2} \text { Trace }\left[C^{-1} \frac{\partial C}{\partial \theta_{i}} C^{-1} \frac{\partial C}{\partial \theta_{j}}\right] .
$$

\subsection{Future $C M B$ experiments}

The quality of the data from the WMAP satellite $[55,65]$, complemented by the results of other experiments at smaller angular scales such as those listed 
in section 5.2, has shown the importance of CMB data as a probe of cosmological parameters. There are several other projects in operational or design stage, aimed mainly at improving the sensitivity towards small scales and/or polarization anisotropies. The neutrino mass is not the primary target of these experiments, because as we have already seen in Sec. 4.6, small masses only have a modest signature on the CMB. But still, we know that there is a background effect proportional to $\omega_{\nu}$ which renders the primary CMB anisotropies sensitive -at least in principle- to very small values of the total neutrino mass. Instead, typical targets are the scalar tilt and the tensor-to-scalar ratio, in order to better constrain the predictions of inflationary theories. However, in order to measure with precision the neutrino masses, it will be useful to combine some large scale structure observations with the best possible CMB dataset in order to reduce as much as possible parameter degeneracies. In this section we review the Fisher matrix computation and expected sensitivity of future CMB experiments alone; in the next sections the CMB contribution to the Fisher matrix will be combined with that from other hypothetical observations.

The CMB temperature maps can be decomposed in multipoles

$$
\frac{\Delta T}{T}(\theta, \phi)=\sum_{l, m} a_{l m}^{T} Y_{l m}(\theta, \phi),
$$

and similarly the polarization map are described by multipoles $a_{l m}^{E}$ and $a_{l m}^{B}$. The data covariance matrix $C=\left\langle a_{l m}^{X} a_{l^{\prime} m^{\prime}}^{X^{\prime}}\right\rangle$ receives contributions from:

- primary anisotropies, for which the expected variance is predicted by the cosmological perturbation theory: $\left\langle a_{l m}^{X} a_{l^{\prime} m^{\prime}}^{X^{\prime}}\right\rangle=\delta_{l l^{\prime}} \delta_{m m^{\prime}} C_{l}^{X X^{\prime}}$. For parity reasons the non-vanishing power spectra are $C_{l}^{T T}, C_{l}^{E E}, C_{l}^{B B}$ and $C_{l}^{T E}$.

- secondary anisotropies imprinted at redshifts $z \ll 1100$, like the late Integrated Sachs-Wolfe (ISW) effect and the weak lensing induced by nearby clusters, as well as various foreground contaminations. The late ISW effect is usually included in the theoretical power spectrum computation, while the lensing effect is small enough to give a negligible contribution to $C$ (excepted for the small-scale B-mode variance). Here we will neglect the lensing distortions, which will be the object of Sec. 6.4. Most of the foreground contamination is expected to be accurately removed from the raw data using a multi-frequency analysis (since most foreground signals do not have a blackbody distribution).

- experimental noise, which is usually parametrized by two quantities: the angular resolution and the sensitivity per pixel (here, a pixel represents the smallest area in a map on which an independent measurement is performed). Intuitively, a given angular resolution implies that the error-bar will grow 
dramatically below a given scale, and the finite sensitivity per pixel implies that even in absence of a primary signal, the covariance matrix would get some non-zero contribution at a given level. Technically, the angular resolution is expressed as an angle $\theta_{b}$, the Full-Width at Half-Maximum (FWHM) of the instrument beam. The sensitivity (or noise squared variance) per pixel $\Delta_{X}^{2}$ (for $X=T, E, B$ ) is usually given in units of a squared temperature, and the dimensionless sensitivity per pixel $\bar{\Delta}_{X}^{2}$ is obtained dividing by the square of $T_{0} \simeq 2.726 \mathrm{~K}$. The solid angle of a pixel can be approximated by $\theta_{b}^{2}$, so that the dimensionless sensitivity per steradians reads $\left(\theta_{b}^{2} \bar{\Delta}_{X}^{2}\right)$. For one instrument, the noise variance can be shown to be [194]

$$
\left\langle a_{l m}^{X} a_{l^{\prime} m^{\prime}}^{X^{\prime}}\right\rangle=\delta_{l l^{\prime}} \delta_{m m^{\prime}} \delta^{X X^{\prime}} N_{l}^{X X}, \quad N_{l}^{X X} \equiv\left(\theta_{b}^{2} \bar{\Delta}_{X}^{2}\right) B_{l}^{-2},
$$

where the Gaussian beam window function $B_{l} \equiv \exp \left[-l(l+1) \theta_{b}^{2} /(16 \ln 2)\right]$ has the effect of cutting the sensitivity for $l \gg \theta_{b}^{-1}$. Note that for $X \neq X^{\prime}$ the variance vanishes, since the noise in one map (for mode $T, E$ or $B$ ) is expected to be uncorrelated with the noise in another map. Finally, most experiments consist of various detectors, for which a global sensitivity can be built by summing the inverse variances

$$
\left\langle a_{l m}^{X} a_{l^{\prime} m^{\prime}}^{X^{\prime}}\right\rangle=\left(\sum_{i}\left[\theta_{b}^{-2} \bar{\Delta}_{X}^{-2} B_{l}^{2}\right]_{i}\right)^{-1}
$$

Detectors are generally grouped in channels, i.e. ensembles of detectors working at a given frequency. An efficient foreground extraction requires a large number of channels, at the expense of decreasing the number of detectors per channel, and hence increasing the noise per channel.

The theoretical and noise variance are usually assumed to be uncorrelated, which allows to express the covariance matrix in a rather simple way. If we represent the data as a vector $\vec{x}=\mathbf{x}_{l m}=\left(a^{T}, a^{E}, a^{B}\right)_{l m}$, where the indexes $(l, m)$ run over all multipoles, the full data covariance matrix can be decomposed in diagonal blocks $C_{l m}$ representing the covariance $\left\langle\mathbf{x}_{l m} \mathbf{x}_{l m}^{t}\right\rangle$ for a given $(l, m)$

$$
C_{l m}=C_{l}=\left(\begin{array}{ccc}
C_{l}^{T T}+N_{l}^{T T} & C_{l}^{T E} & 0 \\
C_{l}^{T E} & C_{l}^{E E}+N_{l}^{E E} & 0 \\
0 & 0 & C_{l}^{B B}+N_{l}^{B B}
\end{array}\right) .
$$

The index $m$ does not appear in this matrix, since all multipoles $(l, m)$ with the same $l$ share the same variance. If we consider the data as Gaussian (which is a very good approximation except for small-scale $B$-mode multipoles), we can use Eq. (149). The first step of the calculation is to estimate the number of multipoles $(l, m)$ with a fixed $l$. For full-sky experiments, $m$ runs from $-l$ 
to $l$ and this number is $(2 l+1)$. For experiments probing a fraction $f_{\text {sky }}$ of the sky, the number of independent multipole measurement at a given $l$ is close to $(2 l+1) f_{\text {sky }}$. This leads to

$$
F_{i j}^{\mathrm{CMB}}=\frac{1}{2} \sum_{l=2}^{l_{\max }}(2 l+1) f_{\mathrm{sky}} \text { Trace }\left[C_{l}^{-1} \frac{\partial C_{l}}{\partial \theta_{i}} C_{l}^{-1} \frac{\partial C_{l}}{\partial \theta_{j}}\right]
$$

which can be further reduced to an expression of the type [195]

$$
F_{i j}^{\mathrm{CMB}}=\sum_{l=2}^{l_{\max }} \sum_{\alpha, \beta} \frac{\partial C_{l}^{\alpha}}{\partial \theta_{i}}\left(\operatorname{Cov}_{l}\right)_{\alpha \beta}^{-1} \frac{\partial C_{l}^{\beta}}{\partial \theta_{j}}
$$

where $\alpha$ and $\beta$ run over TT, EE, TE, BB and $\operatorname{Cov}_{l}$ is the "spectra covariance matrix". For space reason we do not give here the full expression of $\operatorname{Cov}_{l}$ (see e.g. Ref. [196]). As an example, the first coefficient of the matrix reads

$$
\left(\operatorname{Cov}_{l}\right)_{T T, T T}=\frac{2}{(2 l+1) f_{\text {sky }}}\left(C_{l}^{T T}+N_{l}^{T T}\right)^{2} .
$$

The factor $(2 l+1) f_{\text {sky }}$ which appears in the denominator of $\operatorname{Cov}_{l}$ accounts for the effect of cosmic variance: for large $l$ values, an experiment makes many independent measurements (corresponding to several $m$ 's) and the variance in absence of noise is much smaller than the power spectrum $C_{l}^{X X}$ of a single multipole $a_{l m}^{X}$. For small $l$, there are only few independent measurement and the variance is of the same order of magnitude as $C_{l}^{X X}$.

Let us apply this machinery to concrete cases. In Table 4, we present the expected sensitivity of a non-exhaustive list of future CMB experiments. The design of projected experiments is subject to constant evolution, and this compilation should be regarded as indicative only. We did not take the numbers from some official documentation edited by each experiment, but from the papers cited below, which worked out some neutrino mass error forecasts on the basis of the numbers presented here. We refer to these papers for more details and references concerning the sensitivity parameters.

After WMAP, we can expect some very interesting results from a large number of ground-based experiments, mapping the anisotropies only in a small regions of the sky but usually with excellent sensitivity and resolution. Most of these experiments are optimized for measuring E-polarization or even B-polarization anisotropies, which will still be poorly constrained after the completion of WMAP, and even after PLANCK in the case of B-polarization. Among these collaborations, we can cite the South Pole Telescope ${ }^{8}$ (SPT, in construction)

$8 \overline{\text { http://spt.uchicago.edu/ }}$ 
Table 4

Experimental parameters of a few CMB projects, used in the neutrino mass forecast papers mentioned in this section. Here $f_{\text {sky }}$ is the observed fraction of the sky, $\nu$ is the channel frequency in $\mathrm{GHz}, \theta_{b}$ measures the FWHM of the beam in arc-minutes and $\Delta_{T, P}$ is the square root of the sensitivity per pixel in $\mu \mathrm{K}$ for temperature and polarization.

\begin{tabular}{|c|c|c|c|c|c|}
\hline Experiment & $f_{\text {sky }}$ & $\nu$ & $\theta_{b}$ & $\Delta_{T}$ & $\Delta_{P}$ \\
\hline SPT [197] & 0.1 & 217 & $0.9^{\prime}$ & 12 & 17 \\
\hline \multirow[t]{2}{*}{ BICEP [198] } & 0.03 & 100 & $60^{\prime}$ & 0.33 & 0.47 \\
\hline & & 150 & 42 & 0.35 & 0.49 \\
\hline \multirow[t]{2}{*}{ QUaD [198] } & 0.025 & 100 & $6.3^{\prime}$ & 3.5 & 5.0 \\
\hline & & 150 & $4.2 '$ & 4.6 & 6.6 \\
\hline \multirow[t]{3}{*}{ BRAIN [198] } & 0.03 & 100 & $50^{\prime}$ & 0.23 & 0.33 \\
\hline & & 150 & 50 & 0.27 & 0.38 \\
\hline & & 220 & 50 & 0.40 & 0.56 \\
\hline \multirow[t]{3}{*}{ ClOVER [198] } & 0.018 & 100 & 15 & 0.19 & 0.30 \\
\hline & & 143 & 15 & 0.25 & 0.35 \\
\hline & & 217 & $15^{\prime}$ & 0.55 & 0.76 \\
\hline \multirow[t]{9}{*}{ PLANCK [198] } & 0.65 & 30 & 33 & 4.4 & 6.2 \\
\hline & & 44 & 23 & 6.5 & 9.2 \\
\hline & & 70 & $14^{\prime}$ & 9.8 & 13.9 \\
\hline & & 100 & 9.5 & 6.8 & 10.9 \\
\hline & & 143 & $7.1^{\prime}$ & 6.0 & 11.4 \\
\hline & & 217 & $5.0^{\prime}$ & 13.1 & 26.7 \\
\hline & & 353 & $5.0^{\prime}$ & 40.1 & 81.2 \\
\hline & & 545 & $5.0^{\prime}$ & 401 & $\infty$ \\
\hline & & 857 & $5.0^{\prime}$ & 18300 & $\infty$ \\
\hline \multirow[t]{3}{*}{ SAMPAN [198] } & 0.65 & 100 & $42^{\prime}$ & 0.13 & 0.18 \\
\hline & & 143 & $30^{\prime}$ & 0.16 & 0.22 \\
\hline & & 217 & $20^{\prime}$ & 0.26 & 0.37 \\
\hline CMBpol [197] & 0.65 & 217 & $3.0^{\prime}$ & 1 & 1.4 \\
\hline \multirow{4}{*}{$\begin{array}{l}\text { Inflation Probe } \\
\text { (hypothetical) [198] }\end{array}$} & 0.65 & 70 & $6.0^{\prime}$ & 0.29 & 0.41 \\
\hline & & 100 & 4.2 & 0.42 & 0.59 \\
\hline & & 150 & 2.8 & 0.63 & 0.88 \\
\hline & & 220 & $1.9^{\prime}$ & 0.92 & 1.30 \\
\hline
\end{tabular}


[199], the Atacama Cosmology Telescope ${ }^{9}$ (ACT, funded in January 2004), and two complementary experiments: Background Imaging of Cosmic Extragalactic Polarization ${ }^{10}$ (BICEP) [200], designed for large angular scale, and QUest at DASI ${ }^{11}$ (QUaD) [201], designed for small angular scales. The second experiment, which is already collecting data, is composed of the $\mathrm{Q}$ and $\mathrm{U}$ Extragalactic Sub-mm Telescope (QUEST) instrument mounted on the structure of the DASI experiment. A second set of experiments is scheduled in Antarctica at the French-Italian Concordia station and in the Atacama plateau in Chile, for unprecedented precision measurements of the $B$-mode for $l<1000$ (which is particularly useful for probing the primordial gravitational waves from inflation): the B-modes Radiation measurement from Antarctica with a bolometric INterferometer ${ }^{12}$ (BRAIN) [202] instrument for measuring large scales, and the Cl ObserVER ${ }^{13}$ (ClOVER) [203] instrument for intermediate scales. ClOVER was approved for funding by PPARC in late 2004 and could be operational by 2008 .

At that time, a CMB satellite (the third one after COBE and WMAP) should be already collecting data: the PLANCK ${ }^{14}$ satellite [204] has already been built and should be launched in late 2007 or early 2008 by the European Space Agency (ESA). The temperature sensitivity of PLANCK will be so good that it can be thought as the "ultimate" observation in the sector of temperature anisotropies. However, it will still be possible to improve the measurement of E-polarization on small angular scales, and B-polarization will be poorly constrained by PlancK. On intermediate scales, the ground-based experiments should be quite efficient, but progress will still be needed on very large scales (requiring full-sky coverage) and very small scales (requiring both high resolution and excellent sensitivity). Beyond PLANCK, at least two space projects are under investigation. The mini-satellite SAMPAN (SAtellite to Measure the Polarized ANisotropies) [205] is targeted for large scales, and aims at improving the ClOVER measurement of the $B$-mode for $l<1000$, thanks to its full-sky coverage and slightly better sensitivity. The NASA wishes to launch a more ambitious satellite project in order to make the "ultimate" measurement of E-polarization, like PLANCK for temperature, and a very good measurement of B-polarization on all scales, with for the first time an instrumental noise smaller than the B-mode cosmic variance up to $l \sim 1000$ or even maybe $l \sim 1500$. The generic name of the NASA call for projects is INFLATION PROBE ${ }^{15}$. Its design is still very uncertain. A very preliminary project

\footnotetext{
$9 \overline{\text { http: //www.hep.upenn.edu/ angelica/act/act.html }}$

10 http://www . astro.caltech.edu/ lgg/bicep_front.html

11 http://www.astro.cf.ac.uk/groups/instrumentation/projects/quad/

12 http://apc-p7.org/APC_CS/Experiences/Brain/index.phtml

13 http://www-astro.physics.ox.ac.uk/ act/clover.html

14 http://sci.esa.int/science-e/www/area/index.cfm?fareaid=17

15 http://universe.gsfc.nasa.gov/program/inflation.html
} 
was called CMBpol; this name is mentioned in some works presented thereafter $[197,206,101]$, with sketchy characteristics mentioned in Table 4 . There are now more advanced projects under investigation. Nobody knows which project and which design will be eventually approved. We mention in the Table some hypothetical characteristics for Inflation ProBe suggested in Ref. [198], assuming a satellite project with a bolometer array and a passively cooled telescope of 3-4 $\mathrm{m}$ of aperture. This is one of the various possibilities which are being discussed, and it should be regarded as purely indicative.

Some predictions concerning the sensitivity of these experiments (not combined with other observations) to the neutrino mass are published in Refs. $[196,207,101,198]$ for PLANCK and in Ref. [198] for the combinations BICEP + QUaD, BRAIN+ClOVER, as well as SAMPAN alone, PLANCK+SAMPAN and the hypothetical version of INFLATION PROBE (see also [208,209] for forecasts including a large neutrino asymmetry, now disfavoured as we saw in Sec. 3.1). Like for current bounds, forecast errors are not straightforward to compare from paper to paper, because different authors use

- different fiducial models with different fiducial values for the cosmological parameters. In Ref. [196], the fiducial model which is closer to the current concordance $\Lambda$ CDM model is the one in Table II of that paper; here, we will only quote results from this table. There are eleven free parameters, which are the usual six of the $\Lambda \mathrm{CDM}$ model, the mass of a single massive neutrino, the tensor-to-scale ratio $T / S$, the scalar tilt running $\alpha$, the curvature fraction $\Omega_{k}$ and the ionized helium fraction $Y_{\mathrm{He}}$. The fiducial value of $M_{\nu}$ is very close to zero (so that the fiducial CMB spectra are indistinguishable from those with massless neutrinos). In Ref. [207], the number of free parameters is reduced to eight, by removing $\left(T / S, \alpha, Y_{\mathrm{He}}\right)$ which are fixed respectively to $(0,0,0.24)$. There is a single massive neutrino with fiducial mass $0.07 \mathrm{eV}$. Next, in Table III of Ref. [101], there are also eight free parameters, but the last one is $Y_{\mathrm{He}}$ instead of $\Omega_{k}$. There are three massive neutrinos with total mass $M_{\nu}=0.3 \mathrm{eV}$ distributed according to the normal hierarchy scheme. Finally, in Table II of Ref. [198], there authors marginalize either over eight parameters (the usual six of $\Lambda$ CDM model, the mass of a single massive neutrino and $Y_{\mathrm{He}}$ ) or over eleven parameters (the same ones, plus the scalar tilt running $\alpha$, the parameter $w$ of the equation of state of dark energy, and the effective number $N_{\text {eff }}$ of extra relativistic degrees of freedom). The fiducial value of the neutrino mass is $M_{\nu}=0.1 \mathrm{eV}$.

- different experimental sensitivities. For instance, the modelling of PLANCK in Refs. [196], [101] and [198] are roughly equivalent, although in [101,198] it was updated to the current instrument design. Instead, in Ref. [207], the author replaces the expression of the noise variance $N_{l}^{T T}, N_{l}^{E E}$ [see Eq. (151)] by two step functions equal to zero below some critical multipoles $\left(l_{\max }^{T}\right.$, $\left.l_{\max }^{E}\right)=(2500,1500)$ and to infinity above. This is equivalent to assuming a 
Table 5

Forecast error $\sigma\left(M_{\nu}\right)$ in eV using PLANCK alone: compared results from the literature. The third column shows the free parameters over which the final result is marginalized ("7" stands for six usual parameters of the minimal $\Lambda$ CDM model, plus total neutrino mass $M_{\nu}$ ). The fourth column gives the assumed fiducial value of the mass in $\mathrm{eV}$. In the last two lines, the two numbers correspond to optimistic or pessimistic assumptions concerning the foregrounds contamination of the primary CMB signal.

\begin{tabular}{ccccc}
\hline Ref. & $\sigma\left(M_{\nu}\right)$ & parameters & fiducial $M_{\nu}$ & PLANCK sensitivity \\
\hline$[196]$ & 0.3 & $7+\left\{\alpha, T / S, \Omega_{\mathrm{k}}, Y_{\mathrm{He}}\right\}$ & 0 & slightly optimistic \\
{$[207]$} & 0.07 & $7+\left\{\Omega_{\mathrm{k}}\right\}$ & 0.07 & very optimistic \\
{$[101]$} & 0.3 & $7+\left\{Y_{\mathrm{He}}\right\}$ & 0.3 & up-to-date \\
{$[198]$} & $0.45-0.49$ & $7+\left\{Y_{\mathrm{He}}\right\}$ & 0.1 & up-to-date \\
{$[198]$} & $0.51-0.56$ & $7+\left\{\alpha, w, N_{\mathrm{eff}}, Y_{\mathrm{He}}\right\}$ & 0.1 & up-to-date \\
\hline
\end{tabular}

slightly over-optimistic version of PLANCK.

- different parameter basis and different finite steps for the computation of the derivatives $\partial C_{l}^{X X^{\prime}} / \partial \theta_{i}$. The likelihood $\mathcal{L}(\vec{x} \mid \vec{\theta})$ can be close to a multivariate Gaussian in one basis, and more complicated in another basis - in which case Eq. (155) may give only a poor approximation of the Fisher matrix. The possible non-gaussianity also implies that the numerical value of the derivative $\partial C_{l}^{X X^{\prime}} / \partial \theta_{i}$ depends on the step $\Delta \theta_{i}$ used for its calculation. However, authors usually optimize their choice of basis and step size [see Ref. [196])] in order to obtain robust results.

In order to illustrate how these different assumptions are translated into different forecasts from paper to paper, we summarize in Table 5 the predictions for PLANCK published by the four references $[196,207,101,198]$. In the case of Ref. [198] we quote two numbers, which represent some optimistic or pessimistic assumptions concerning the subtraction of astrophysical foregrounds from the observed CMB maps: these two values, which are not very different from each other, are supposed to bracket the true realistic $1 \sigma$ error. The difference between $\sigma\left(M_{\nu}\right)=0.3 \mathrm{eV}$ in [196] and $\sigma\left(M_{\nu}\right)=0.07 \mathrm{eV}$ in [207] (taken from Fig. 3 of this reference) probably comes from the sketchy and very optimistic modelling of the noise in the second reference. The results of [196] and [101] seem to be consistent with each other, since in the latter reference the number of parameters is smaller but the experimental characteristics are more realistic. The difference between $\sigma\left(M_{\nu}\right)=0.3 \mathrm{eV}$ in [101] and $\sigma\left(M_{\nu}\right) \simeq 0.45 \mathrm{eV}$ in [198], for identical cosmological models and experimental characteristics, comes from variations in the fiducial value of the total neutrino mass, which decreases from 0.3 to $0.1 \mathrm{eV}$. Indeed, Ref. [101] explores the dependence of 
Table 6

Forecast error $\sigma\left(M_{\nu}\right)$ in eV using various CMB experiments or combinations of them. These numbers are all taken from Ref. [198], which assumes either an eight parameter model (the usual six ones of $\Lambda \mathrm{CDM}$ plus $M_{\nu}$ and $Y_{\mathrm{He}}$ ), or an eleven parameter model (adding $\alpha, w$ and $N_{\text {eff }}$ ). The fiducial value of the total mass is taken to be $M_{\nu}=0.1 \mathrm{eV}$, and in each case the two numbers correspond to optimistic or pessimistic assumptions concerning the foregrounds contamination of the primary CMB signal.

\begin{tabular}{ccc}
\hline Experiment & 8 parameters & 11 parameters \\
\hline BICEP+QUaD & $1.3-1.6$ & $1.5-1.9$ \\
BRAIN+ClOVER & $1.5-1.8$ & $1.7-2.0$ \\
PLANCK & $0.45-0.49$ & $0.51-0.56$ \\
SAMPAN & $0.34-0.40$ & $0.37-0.44$ \\
PLANCK+SAMPAN & $0.32-0.36$ & $0.34-0.40$ \\
INFLATION PROBE & $0.14-0.16$ & $0.25-0.26$ \\
\hline
\end{tabular}

$\sigma\left(M_{\nu}\right)$ on the fiducial value $M_{\nu}$ (see Fig. 22), and find that when $M_{\nu}$ decreases from approximately 0.5 to $0.1 \mathrm{eV}, \sigma\left(M_{\nu}\right)$ is multiplied roughly by a factor two. Finally, the comparison of the two results obtained in Ref. [198] shows that the determination of the neutrino mass by PLANCK alone does not suffer significantly from parameter degeneracies which appear when $\alpha, w$ or $N_{\text {eff }}$ are included as extra free parameters. Similarly, the comparison of [196] and [101] suggests that this also applies for the possible inclusion of $T / S$ and $\Omega_{\mathrm{k}}$.

For other CMB experiments than PLANCK, not combined with other type of observations, the only available reference is [198], whose results are summarized in Table 6 . We see in this table that ground based experiments alone are not helpful for constraining the neutrino mass: this is due to that fact that with a small sky coverage, and in spite of their excellent sensitivity, they have relatively large error-bars (with respect to PLANCK) for small and intermediate values of $l$. We will see in Sec. 6.4 how this conclusion changes when one assumes that the results of these experiments are analyzed with the "lensing extraction" technique.

In summary, the satellites PLANCK, SAMPAN or the combination of both would reach a $1 \sigma$ error in the range $\sigma\left(M_{\nu}\right)=0.3-0.4 \mathrm{eV}$, depending on the case. This is of the same order of magnitude as the current neutrino mass bounds presented in section 5, when all available CMB and LSS data are combined with each other. Finally, if the Inflation probe mission can actually reach the sensitivity assumed in Table 4, it will provide a sensitivity ranging from $\sigma\left(M_{\nu}\right)=0.15 \mathrm{eV}$ to $0.25 \mathrm{eV}$ depending on the number of marginalized parameters. 


\subsection{Future galaxy redshift surveys}

As already discussed in Sec. 5.3, Galaxy Redshift Surveys (GRS) allow for a reconstruction of the matter power spectrum modulo a light-to-mass bias factor. The analytic models for non-linear structure formation show that the relation between the reconstructed power spectrum and the total matter linear power spectrum should be scale-independent, $P_{\text {obs }}(k) \equiv b^{2} P(k)$, at least up to a wavenumber $k_{\max }$ where non-linear corrections start to induce scaledependent biasing. At redshift zero and for the concordance $\Lambda$ CDM model, this wavenumber is expected to be around $k_{\max }=0.15 h \mathrm{Mpc}^{-1}$. The usual approach is to discard any information for $k>k_{\max }$ in order to avoid many complications related to biasing, non-Gaussian statistics and uncertainties in the predictions of the non-linear matter power spectrum.

A Fisher matrix can be derived for future surveys following the lines of Sec. 6.1. It was shown by Tegmark [210] that the Fisher matrix takes a very simple form under a couple of assumptions.

- For $k \leq k_{\max }$, one assumes that the power spectrum reconstructed from the data, $P_{\text {obs }}(k)$, is really a tracer of the total matter power spectrum, not significantly affected by non-linear corrections or by redshift-space distortions. We recall that what is measured is the redshift of each galaxy, not its physical distance; therefore, strictly speaking, the power spectrum is not reconstructed in three-dimensional real space but in redshift space; the redshift-space and real-space power spectra do not coincide exactly, they are related through a function which depends on the cosmological parameters, and this complication is ignored here in first approximation.

- One assumes that the survey is volume-limited rather than brightnesslimited, i.e. the spectrum is reconstructed from data within a volume $V$ in which the selection function $n(r)$, which represents the number density of observed galaxies, is approximately constant (instead of being suppressed near the edges due to the fact that only the brightest galaxies would be seen). Moreover, one assumes that the density is large enough so that $1 / n(r) \leq P(k)$ for all relevant wavenumbers $k_{\min }<k<k_{\max }$, which means that the galaxy correlation function really probes cosmological information rather than shot noise due to insufficient sampling.

Under these assumptions, the Fisher matrix takes a form which is analogous to its CMB counterpart of Eq. (155):

$$
F_{i j}^{\mathrm{GRS}}=\frac{1}{2} \int_{k_{\min }}^{k_{\mathrm{max}}} \frac{\partial P_{\mathrm{obs}}(k)}{\partial \ln \theta_{i}} \frac{\partial P_{\mathrm{obs}}(k)}{\partial \ln \theta_{j}} \frac{4 \pi w(k)}{P_{\mathrm{obs}}^{2}(k)} d \ln k,
$$


where the weight function $w(k)$ of the survey is defined as

$$
w(k)=\frac{V}{\lambda^{3}}=\frac{V}{(2 \pi / k)^{3}} .
$$

In Eq. (157), the factor $4 \pi w(k) d \ln k$ accounts for cosmic (or sampling) variance: it gives the number of independent measurements with wavenumber in the range $[k, k+d k]$ within the survey volume. This factor is negligible for wavenumbers smaller than $k_{\min }$, the smallest wavenumber probed by the survey: so, for practical purposes, the integral can be performed starting from zero. The result of Ref. [210] is actually more general than above, and includes the case where shot noise is not negligible and where the selection function is not constant inside the volume $V$. In these cases, one must replace $V$ in Eqs. (157) and (158) by an effective volume $V_{\text {eff }}(k)$ defined as

$$
V_{\mathrm{eff}}(k)=\int d^{3} \mathbf{r}\left[\frac{P_{\mathrm{obs}}(k)}{1 / n(\mathbf{r})+P_{\mathrm{obs}}(k)}\right]^{2} .
$$

However, for the future surveys discussed in this section, we can systematically assume that the power spectrum reconstruction will be performed inside a volume $V$ chosen in such way that throughout this volume $1 / n(\mathbf{r})$ is smaller than $P_{\text {obs }}(k)$ for all $k_{\min }<k<k_{\max }$, so that $V_{\text {eff }}(k)$ can be replaced by $V$.

For instance, for the completed SDSS Bright Red Galaxy (BRG) survey, this volume will include galaxies up to a distance $\lambda \simeq 1 h^{-1} \mathrm{Gpc}$ with a sky coverage $f_{\text {sky }}=0.25$. So, one can take $V \sim f_{\text {sky }}(4 / 3) \pi \lambda^{3}=1(\mathrm{Gpc} / h)^{3}[196]$. Beyond SDSS, there are various plans for larger surveys, like for instance the SDSS-II LEGACY project, the Advanced Large, Homogeneous Area Medium Band Redshift Astronomical survey ${ }^{16}$ (ALHAMBRA) [211], the Dark Energy Survey $^{17}$ (DES) $[212,213,214]$, or the Kilo-Aperture Optical Spectrograph ${ }^{18}$ (KAOS). Some of these surveys will go to such high redshift that it will be possible, first, to compare the observed power spectrum with the linear one up to wave-numbers significantly larger than the usual $k_{\max } \sim 0.15 h \mathrm{Mpc}^{-1}$ (since the non-linear evolution affects larger and larger modes as time passes by); and second, to do some tomography, i.e. to reconstruct $P(k)$ in various redshift bins, corresponding to different times in the evolution of the Universe, in order to measure the variations of the linear growth factor and to get some good handle on the dark energy variables. Tomography is also ideal for neutrino mass extraction, since massive neutrinos induce a very peculiar redshift-dependence on the matter power spectrum, as we have seen in

\footnotetext{
$\overline{16}$ http://alhambra.iaa.es:8080/

17 http://www . darkenergysurvey.org/

18 http://www.noao.edu/kaos
} 
Sec. 4.6.4. For instance, KAOS could build two catalogs centered around redshifts $z=1$ and $z=3$, corresponding roughly to $k_{\max } \sim 0.2 h \mathrm{Mpc}^{-1}$ and $k_{\text {max }} \sim 0.48 h \mathrm{Mpc}^{-1}$ respectively, instead of $k_{\max } \sim 0.1 h \mathrm{Mpc}^{-1}$ for current surveys (conservative values). In both catalogs, the number density would be such that $1 / n \sim P\left(k_{\max }\right)$, and the effective volume of the two samples close to $V \sim 0.5(\mathrm{Gpc} / h)^{3}$ and $V \sim 0.6(\mathrm{Gpc} / h)^{3}$ respectively ${ }^{19}$.

In order to forecast the sensitivity of a galaxy survey combined with a CMB experiment, one can simply add the two Fisher matrices and invert their sum.

The sensitivity of large redshift surveys to the neutrino mass was fist studied in Ref. [94]; however this pioneering paper was rather crude in the way to combine GRS with CMB experiments. This work was updated and generalized in [196], and later in $[207,101,102]$. In Table 7 , we quote from these papers the forecast error $\sigma\left(M_{\nu}\right)$ obtained assuming that the completed SDSS survey is combined either with the WMAP or PLANCK data. These results are based on Table II in [196], Figs. 4 and 5 in [207], and Table III in [101], for a common conservative value $k_{\max }=0.1 \mathrm{~h} \mathrm{Mpc}^{-1}$. With respect to the results quoted in the previous subsection, the number of free parameters has increased by one unit, since there is now an unknown free bias parameter $b=\left[P_{\text {obs }}(k) / P(k)\right]^{1 / 2}$ which is marginalized out. We see that SDSS alone gives essentially no information on $M_{\nu}$ given the various parameter degeneracies. For the SDSS+WMAP combination, there is an excellent agreement between [196] and [207], and the forecast error $\sigma\left(M_{\nu}\right)=0.3 \mathrm{eV}$ is three times smaller than the current error based on incomplete WMAP+SDSS results $\sigma\left(M_{\nu}\right)=0.9 \mathrm{eV}$ [135]. For SDSS+PLANCK, it seems again that the PLANCK noise modelling in [207] is much too optimistic, while Refs. [196] and [101] agree on $\sigma\left(M_{\nu}\right)=0.2 \mathrm{eV}$. If we compare this with the result obtained in [101] under the same assumptions for PLANCK alone, $\sigma\left(M_{\nu}\right)=0.3 \mathrm{eV}$, we see that SDSS allows for a significant improvement.

Concerning experiments beyond SDSS, Ref. [207] and later Ref. [101] present forecast errors in a very general way, by simply showing $\sigma\left(M_{\nu}\right)$ as a function of two free parameters $V$ and $k_{\max }$. Instead Ref. [102] assumes some detailed characteristics for three high-redshift surveys, each of them operating in three redshift bins. Here we will summarize the results of Ref. [101], assuming that an hypothetical future redshift survey with given $\left(V, k_{\max }\right)$ is combined with a CMB experiment that could be SPTpol, PlAnCK, CMBpol (see Table 4 for the value of assumed sensitivity parameters), or an "ideal CMB experiment" that would be limited only by cosmic variance up to $l=2500$ (both for temperature and polarization). The main difficulty for reaching this goal would be to subtract accurately small-scale foregrounds (point-like sources,

$\overline{19}$ The characteristics of KAOS are taken from the "Purple Book" available on-line at http://www.noao.edu/kaos 
Table 7

Forecast error $\sigma\left(M_{\nu}\right)$ in $\mathrm{eV}$ for the completed SDSS survey, with volume $V=$ $1(\mathrm{Gpc} / h)^{3}$, eventually combined with WMAP or PLANCK data: compared results from the literature, assuming the conservative value $k_{\max }=0.1 \mathrm{~h} \mathrm{Mpc}^{-1}$.

\begin{tabular}{ccccc}
\hline Ref. & SDSS & +WMAP & +Planck & assumptions \\
\hline$[196]$ & 9 & 0.3 & 0.2 & $\begin{array}{c}12 \text { free parameters, } \\
\text { optimistic PLANCK characteristics }\end{array}$ \\
\hline$[207]$ & - & 0.3 & 0.06 & 9 free parameters, \\
& & & very optimistic WMAP/Planck charact. \\
\hline$[101]$ & 7 & - & 0.2 & 9 (other) free parameters, \\
& & & & up-to-date PLANCK characteristics \\
\hline
\end{tabular}

dusty galaxies, etc.) but even with current technology such an ideal experiment is not unconceivable. For these four cases, Fig. 21 shows the predicted $2 \sigma$ error on the total neutrino mass $M_{\nu}$. In these plots the fiducial value of the total mass was fixed to $M_{\nu}=0.11 \mathrm{eV}$, and distributed according to the NH scheme. For SDSS (or for any survey with $z<1$ ) we expect the relevant value of $k_{\max }$ to be around $0.15 \mathrm{~h} \mathrm{Mpc}^{-1}$. However, depending on the overall amplitude of the matter power spectrum (often parametrized by $\sigma_{8}$, and still poorly constrained) and on future improvements in our understanding of non-linear corrections, this value might appear to be either too optimistic or too pessimistic: this is the reason why it is interesting to leave it as a free parameter. The figure shows the importance of employing high-volume surveys, which have the potential to improved the forecast errors even for the best CMB experiments. Keeping $k_{\max }=0.15 h \mathrm{Mpc}^{-1}$, we see that a two-sigma detection of the minimal mass in the IH scenario would require $V=40(\mathrm{Gpc} / h)^{3}$ after PlanCK, $V=12(\mathrm{Gpc} / h)^{3}$ after CMBpol, while the "ideal CMB experiment" alone would suffice. A two-sigma detection of the minimal mass in the NH scenario seems to be unreachable for any plausible survey volume.

As in the previous subsection, we should mention that the results for $\sigma\left(M_{\nu}\right)$ depend significantly on the assumed fiducial value $M_{\nu}$. This is illustrated in Fig. 22, taken from Ref. [101]. For the combination of either PLANCK with SDSS, or of the "ideal CMB experiment" with a larger hypothetical survey, we can see that the $2 \sigma$ error is typically twice larger for $M_{\nu} \sim 0.1 \mathrm{eV}$ than for $M_{\nu} \sim 0.5 \mathrm{eV}$.

It is legitimate to wonder whether the most precise combination of experiments would be sensitive to different neutrino mass splittings between the three families, for a fixed value of the total mass. Indeed, we have seen in Sec. 4.6 that unlike CMB observables, the power spectrum of large scale structure is in principle sensitive both to the total and the individual values of the 

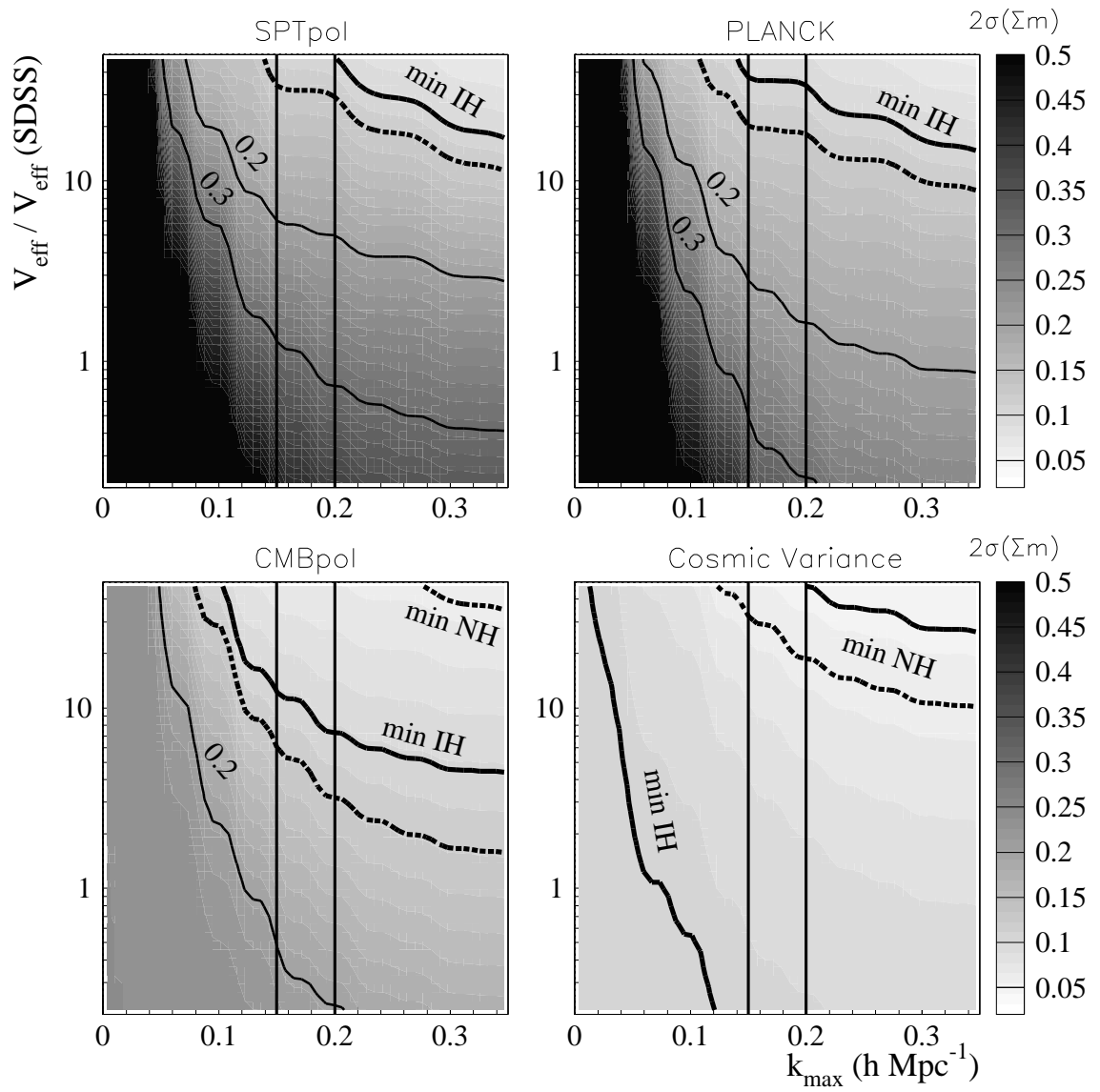

Fig. 21. Results from Ref. [101] on the neutrino mass sensitivity of future redshift surveys, combined with future CMB experiment. The level of gray gives the $2 \sigma$ expected errors on the total mass $M_{\nu}(\mathrm{eV})$ for a fiducial value of $0.11 \mathrm{eV}$, as a function of the largest wavenumber $k_{\max }$ used in the analysis [in units of $h \mathrm{Mpc}^{-1}$ ] and of the survey volume $V$ [in units of $\left.V_{\text {SDSS }}=1(\mathrm{Gpc} / h)^{3}\right]$. The vertical lines indicate the cut-off wavenumber $k_{\max }$ for the linear matter power spectrum at the conservative (optimistic) value $0.15(0.2) \mathrm{h} \mathrm{Mpc}^{-1}$. The thin contours are (from bottom to top) for 0.3 and $0.2 \mathrm{eV}$, while the thick contours correspond to the minimum values of $M_{\nu}$ in the $\mathrm{IH}$ (lower lines) and NH (upper lines) schemes, assuming a squared mass difference $\Delta m_{\text {atm }}^{2}=2.6 \times 10^{-3} \mathrm{eV}^{2}$ (thick solid lines). The thick dashed lines correspond to the same limits for a value $\Delta m_{\mathrm{atm}}^{2}=3.7 \times 10^{-3} \mathrm{eV}^{2}$ which is now essentially ruled out by current data on neutrino oscillations.

three masses. The analysis of Ref. [101] demonstrates however that the tiny difference between the power spectrum obtained in the $\mathrm{NH}$ and $\mathrm{IH}$ cases for a fixed value of the total mass (close to $0.1 \mathrm{eV}$ ) is far too small for being detected even with the combination of a huge redshift survey of volume $V=$ $40(\mathrm{Gpc} / h)^{3}$ with the "ideal CMB experiment".

The issue of degeneracies between the neutrino mass and other parameters, such as those describing the dark energy evolution, probably needs further investigation. Assuming that dark energy has a constant equation-of-state pa- 

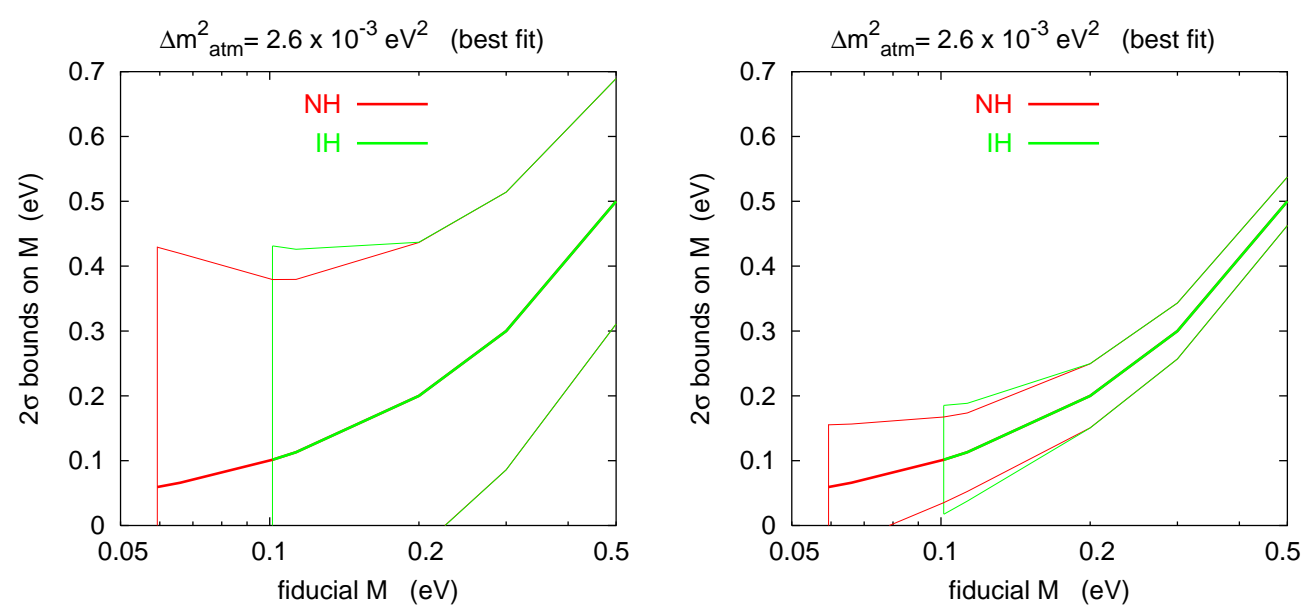

Fig. 22. Predicted $2 \sigma$ error on the total neutrino mass as a function of the assumed fiducial value of $M_{\nu}$, using: (left) PLANCK+SDSS, (right) an ideal CMB experiment (limited only by cosmic variance up to $l=2500$, both for temperature and polarization) and a redshift survey covering $75 \%$ of the sky up to $z \simeq 0.8$ $\left[V_{\text {eff }}=40(\mathrm{Gpc} / h)^{3}\right]$. Both plots assume a limit $k_{\max }=0.15 h \mathrm{Mpc}^{-1}$ and a squared mass difference $\Delta m_{\mathrm{atm}}^{2}=2.6 \times 10^{-3} \mathrm{eV}^{2}$. In each case, we show the results assuming either NH or IH. Figure from Ref. [101].

rameter $w$, Ref. [170] showed that future analyses of combined CMB and LSS data could be affected by a significant degeneracy between $M_{\nu}$ and $w$, a result which was not found in Ref. [101]. The authors of Ref. [86] focused on this degeneracy, including a possible dependence of $w$ on redshift. They point out that in order to help resolving the degeneracy, it is useful to take into account the fact that the CMB and LSS data are not statistically independent, as assumed for simplicity in all the works cited in this section. Instead, it is well-known that due to the late Integrated Sachs-Wolfe (ISW) effect (see Sec. 4.4.7), the power spectrum of temperature anisotropies encodes some information on neighboring structures (like galaxy clusters). So, there is a non-zero cross-correlation between temperature and galaxy maps, which has actually been already measured [215,216], still with a rather low level of significance. In the future, precise measurements of this cross-correlation could be very useful for neutrino mass extraction.

\section{$6.4 \quad C M B$ weak lensing}

\subsubsection{Principle}

It was recently realized that the CMB anisotropies encode even more cosmological information than expected, because it should be possible in a near future to measure the deflection field caused by the weak lensing of CMB photons by the large scale structure of the neighboring universe up to a redshift $z \sim 3[217,218,219]$ (see [220] for a recent review on CMB lensing). The power 
spectrum of the deflection field encodes some information concerning structure formation mainly in the linear or quasi-linear regime, and is therefore extremely useful for measuring parameters like the total neutrino mass (or the dark energy equation-of-state) which mildly affect the primary anisotropy [197]. So, the next generation of CMB experiments could output for free a LSS power spectrum, without suffering from the systematics induced by mass-tolight bias and by strong non-linear corrections on small scales at $z \leq 0.2$, as is the case for galaxy redshift surveys.

More precisely, weak lensing produces a deflection of light rays parametrized by a two-dimensional deflection field $\mathbf{d}(\mathbf{n})$ such that an object located in a direction $\mathbf{n}$ will appear in a direction $\mathbf{n}+\mathbf{d}(\mathbf{n})$. At leading order [221,222], the deflection field can be written as the gradient of a lensing potential, $\mathbf{d}=\nabla \phi$. The lensing potential contains information on the gravitational potential along the photon trajectory, convolved by a window function which depends on the distance to the source

$$
\phi\left(\hat{n} ; r_{s}\right)=\int_{0}^{r_{s}} d r W\left(r ; r_{s}\right) \Phi(r \hat{n}, \eta=-r),
$$

where $r$ is the comoving coordinate along the line of sight, $r_{s}$ is the source coordinate, $\Phi$ the gravitational potential, and $\eta$ the conformal time defined by $d t=-a d \eta$ and $\eta=0$ today (with this definition, the photons emitted by the source and reaching us today travel according to $r(\eta)=-\eta)$. Using the geodesic equation, it is possible to show that the window function $W\left(r ; r_{s}\right)$ is equal to

$$
W\left(r ; r_{s}\right)=2 \frac{r_{s}-r}{r r_{s}}
$$

In the present case, the source is the last scattering surface and $r_{s}$ stands for its comoving radius. For a given cosmological model, the primordial spectrum $\left\langle|\Phi|^{2}\right\rangle$ and its time evolution can be calculated; the same numerical code which computes the CMB anisotropy spectrum $C_{l}^{X X^{\prime}}$ and the matter power spectrum $P(k)$ can also easily compute the deflection field power spectrum $C_{l}^{d d}$, which encodes some information on LSS at redshifts of order $z \sim 3$ or so. Hence, if we could map the CMB deflection field $\mathbf{d}$, we could measure $C_{l}^{d d}$ and compare it with theoretical predictions in order to constrain cosmological parameters.

There are several methods on the market for extracting the deflection map [221,223,224,225], all based on the non-Gaussianity induced by lensing [217]. These methods start from the assumption that both the primary anisotropies and the deflection field are Gaussian; they also assume that the noise present in the temperature and polarization maps is Gaussian and uncorrelated with 
the signal. None of these assumptions is exactly true. Ref. [226] estimated to which extent the lensing extraction will be biased, first, by the non-Gaussianity of the lensing potential caused by the non-linear growth of matter perturbations on small scales, and second, by the imperfect cleaning of the CMB maps from the kinetic Sunyaev-Zel'dovich effect, which also has a blackbody spectrum, induces non-Gaussianity, and features spatial correlations with many of the structures responsible for the lensing. Both effects were found to be relevant (i.e. to induce a significant bias in the estimators). However, they are small enough to preserve the validity of the method. Ref. [227] estimated the impact on lensing extraction of a small level of non-Gaussianity produced in the Early Universe by inflation, reheating or some other mechanisms (for a review, see [228]), and concluded that given the current bounds on primordial non-gaussianity inferred from CMB maps, this non-gaussianity cannot be large enough to threaten lensing extraction.

\subsubsection{Quadratic estimator method}

This field is rather technical, but we can stress the main ideas without entering into many details. For simplicity, we will write the main formulas in the flatsky approximation, which amounts in replacing the expansion of CMB maps in harmonic space by an expansion in two-dimensional Fourier space, and to use two-dimensional vectors 1 instead of pairs of integers $(l, m)$. In the flat-sky approximation the Fourier modes are fully described by the power spectra $\tilde{C}_{l}^{a b}$ where $a$ and $b$ belong to the basis $\{T, E, B\}$ of temperature, $T$-mode polarization and $E$-mode polarization. Weak lensing correlates the lensed multipoles $[229,217]$ according to

$$
\left\langle a(\mathbf{l}) b\left(\mathbf{l}^{\prime}\right)\right\rangle_{\mathrm{CMB}}=(2 \pi)^{2} \delta\left(\mathbf{l}+\mathbf{l}^{\prime}\right) \tilde{C}_{l}^{a b}+f^{a b}\left(\mathbf{l}, \mathbf{l}^{\prime}\right) \phi\left(\mathbf{l}+\mathbf{l}^{\prime}\right)
$$

where the average holds over different realizations (or different Hubble patches) of a given cosmological model with fixed primordial spectrum and background evolution (i.e. fixed cosmological parameters). In this average, the lensing potential $\phi$ is also kept fixed by convention, which makes sense because the CMB anisotropies and the LSS that we observe in our past light-cone are statistically independent, at least as long as we neglect the integrated Sachs-Wolfe effect. The above functions $f^{a b}$ are defined in [224]. For instance, for $a b=T T$ one has

$$
f^{T T}\left(\mathbf{l}, \mathbf{l}^{\prime}\right) C=C_{l}^{T T}\left(\mathbf{l}+\mathbf{l}^{\prime}\right) \cdot \mathbf{l}+C_{l^{\prime}}^{T T}\left(\mathbf{l}+\mathbf{l}^{\prime}\right) \cdot \mathbf{l}^{\prime}
$$

Let us introduce the basic principle of the quadratic estimator method of $\mathrm{Hu}$ \& Okamoto [223,224,225] (which is equivalent in terms of precision to the alternative iterative estimator method of Hirata \& Seljak [221] as long as 
CMB experiments will make noise-dominated measurements of the B-mode, i.e. at least for the next decade). By inverting Eq. (162), one builds a quadratic combination of the temperature and polarization observed Fourier modes

$$
\mathbf{d}^{a b}(\mathbf{L})=\frac{i \mathbf{L} A_{L}^{a b}}{L^{2}} \int \frac{d^{2} \mathbf{l}_{1}}{(2 \pi)^{2}} a\left(\mathbf{l}_{\mathbf{1}}\right) b\left(\mathbf{l}_{\mathbf{2}}\right) g^{a b}\left(\mathbf{l}_{\mathbf{1}}, \mathbf{l}_{\mathbf{2}}\right)
$$

where $\mathbf{l}_{\mathbf{2}}=\mathbf{L}-\mathbf{l}_{\mathbf{1}}$, and in which the normalization condition

$$
A_{L}^{a b}=L^{2}\left[\frac{d^{2} \mathbf{l}_{1}}{(2 \pi)^{2}} f_{T T}\left(\mathbf{l}_{1}, \mathbf{l}_{2}\right) g^{a b}\left(\mathbf{l}_{1}, \mathbf{l}_{2}\right)\right]^{-1}
$$

ensures that $\mathbf{d}^{a b}$ is an unbiased estimator of the lensing potential

$$
\left\langle\mathbf{d}^{a b}(\mathbf{L})\right\rangle_{\mathrm{CMB}}=i \mathbf{L} \phi(\mathbf{L})=\mathbf{d}(\mathbf{L})
$$

Note that, so far, the coefficients $g^{a b}\left(\mathbf{l}_{1}, \mathbf{l}_{2}\right)$ are still arbitrary. From the observed temperature and polarization maps, one could compute each mode of $\mathbf{d}^{a b}$ and obtain various estimates of the deflection modes, precise up to cosmic variance and experimental errors. In order to quantify the total error, it is necessary to compute the power spectra of the quadratic estimators

$$
\left\langle\mathbf{d}^{a b *}(\mathbf{L}) \mathbf{d}^{a b}(\mathbf{L})\right\rangle=(2 \pi)^{2} \delta\left(\mathbf{L}-\mathbf{L}^{\prime}\right) C_{L}^{d d(a b)}
$$

where the average is now taken over both CMB and LSS realizations, since $\phi(\mathbf{L})$ is also a stochastic quantity. In this definition, the power spectra are written with a superscript $d d(a b)$ in order to be distinguished from the actual power spectrum of the true deflection field. These spectra feature the four-point correlation function of the observed (lensed) Fourier modes $\left\langle a\left(\mathbf{l}_{1}\right) b\left(\mathbf{l}_{2}\right) a\left(\mathbf{l}_{3}\right) b\left(\mathbf{l}_{4}\right)\right\rangle$, which should be expanded at order two in $\phi(\mathbf{L})$ in order to catch the leading non-Gaussian contribution.

The four-point correlation functions are composed as usual of a connected and an unconnected piece. The connected piece is by definition a function of the power spectra $C_{l}^{a b}$ in which we now include all sources of variance: cosmic variance, lensing contribution and experimental noise. The unconnected piece is a function of the same spectra plus the deflection spectrum $C_{l}^{d d}$, and as usual it can be decomposed in three terms corresponding to the different pairings of the four indexes [232]: $\left(l_{1}, l_{2}\right),\left(l_{3}, l_{4}\right)$ or $\left(l_{1}, l_{3}\right),\left(l_{2}, l_{4}\right)$ or $\left(l_{1}, l_{4}\right),\left(l_{3}, l_{2}\right)$. The first term leads to considerable simplifications when it is plugged into the expression of the quadratic estimator power spectrum, and the result is simply $C_{l}^{d d}$, as one would expect naively from squaring Eq. (166). The other 
terms lead to more complicated expressions that we will write as a noise term

$$
C_{L}^{d d(a b)}=C_{L}^{d d}+\left[N_{\mathrm{c}}\right]_{L}^{a b}+N_{L}^{a b}
$$

which represent respectively the contribution from the connected piece and from the two non-trivial terms of the unconnected piece [230,232]. In order to get an efficient estimator, one should adopt the set of coefficients $g^{a b}\left(\mathbf{l}_{1}, \mathbf{l}_{2}\right)$ which minimize the noise terms. It is actually much easier to minimize the connected term only, which leads to the simple results

$$
g^{a a}\left(\mathbf{l}_{1}, \mathbf{l}_{2}\right)=\frac{f_{a a}\left(\mathbf{l}, \mathbf{l}^{\prime}\right)}{2 C_{l}^{a a} C_{l^{\prime}}^{a a}} \quad \text { and } \quad\left[N_{\mathrm{c}}\right]_{L}^{a a}=A_{L}^{a a}
$$

for $a=b$ (for $a \neq b$ see [224]). With such a choice, the unconnected piece contribution $N_{L}^{a b}$ can be shown to be smaller than $A_{L}^{a a}$, but not completely negligible [230].

The various estimators $\mathbf{d}^{a b}$ can be constructed for each pair of modes, except for the pair $B B$, because the spectrum $C_{l}^{B B}$ is dominated by lensing at least on small scales, which invalidates the present method. Therefore, the quadratic estimator technique would not be optimal for long-term CMB experiments with cosmic-variance-dominated measurement of the $B$ mode [221,231]. For an experiment of given sensitivity, the five other estimators can be combined into a final minimum variance estimator, which gives the best possible estimate of the deflection field by weighing each estimator accordingly to its noise level. The sensitivity of the PLANCK satellite is slightly above the threshold for successful lensing extraction, but only at intermediate angular scales, and with essentially all the signal coming from the $\mathbf{d}^{T T}$ estimator. The following generation of experiments - such as the CMBpol or Inflation probe project should obtain the lowest noise level from the $\mathbf{d}^{E B}$ estimator [224].

\subsubsection{Neutrino mass from CMB lensing extraction.}

The authors of Refs. [197] and [198] studied the limits that could be obtained on neutrino mass by using future CMB experiments only, assuming that the lensing power spectrum could be extracted using the quadratic estimator technique. In this way, it is possible to combine information on CMB acoustic oscillations and on the surrounding LSS of the Universe at redshifts of order $z \leq 3$, avoiding many of the complicated features of galaxy redshift surveys related to the mass-to-light bias and to the strongly non-linear evolution of small-scale perturbations at small redshift.

The principle of Refs. $[197,198]$ is to treat the power spectrum of primordial anisotropies (for temperature and polarization) and of the deflection field as 
two independent information on the cosmological parameters. The expected error-bars on the temperature and polarization multipoles are estimated from the characteristics of a given CMB experiment, while those on on the lensing multipoles are taken to be the dominant term $\left[N_{\mathrm{c}}\right]_{L}^{a b}$ in Eq. (168), which can be computed from the same experimental characteristics using essentially Eqs. (169) and (165). Then, the authors assume a fiducial model and perform a Fisher matrix analysis in the same fashion as described in the previous subsection.

The fiducial model of Ref. [197] includes ten free parameters: the usual six parameters of the minimal $\Lambda \mathrm{CDM}$ model, the neutrino mass $M_{\nu}$ in the case of three degenerate neutrinos, the equation of state parameter $w$, the running of the tilt $\alpha$ and the primordial Helium abundance $Y_{\mathrm{He}}$. The authors conclude that the PLANCK satellite will output a $1 \sigma$ error-bar $\sigma\left(M_{\nu}\right)=0.15 \mathrm{eV}$, which shows that the use of the lensing extraction technique should improve the global sensitivity in a rather spectacular way at least for this parameter. In the case of the CMBpol project an impressive error of $\sigma\left(M_{\nu}\right)=0.044 \mathrm{eV}$ is predicted.

This study was repeated in Ref. [206], with one extra parameter representing the variation of the dark energy equation-of-state with respect to the scale factor. We saw before that this parameter could potentially introduce extra degeneracies involving the neutrino mass, but the forecast errors are found to be approximately the same: $\sigma\left(M_{\nu}\right)=0.16 \mathrm{eV}$ and $\sigma\left(M_{\nu}\right)=0.046 \mathrm{eV}$ for PLANCK and CMBpol, respectively.

Finally, the authors of [198] assumed two possible fiducial models, one with eighth free parameters (the same as in Ref. [197] with the exception of $\alpha$ and $w$ ) and one with eleven free parameters (same as in Ref. [197] plus the effective number of extra relativistic degrees of freedom $N_{\text {eff }}$ ). They consider six experiments or combinations of experiments as listed in Table 8, and for each case they quote two numbers, corresponding either to optimistic or pessimistic assumptions concerning the subtraction of astrophysical foregrounds from the observed CMB maps.

It can be seen in Table 8 that the combination QUaD+BICEP benefits a lot from lensing extraction, since the error $\sigma\left(M_{\nu}\right)$ decreases from approximately $1.5 \mathrm{eV}$ to at least $0.4 \mathrm{eV}$. Thus, with QUaD+BICEP it should be possible to reach in a near future -using CMB only- the same precision that we have today combining many observations of different types (galaxy-galaxy correlation function, Lyman- $\alpha$ forest) which are affected by various systematics. The situation is almost the same for BRAIN+ClOVER, which should also achieve $\sigma\left(M_{\nu}\right) \sim 0.4 \mathrm{eV}$ using lensing extraction. PLANCK should make a decisive improvement, lowering the error to $\sigma\left(M_{\nu}\right) \sim 0.15 \mathrm{eV}$, in excellent agreement with the results of Ref. [197]. Note that without lensing extraction the error 
Table 8

Forecast error $\sigma\left(M_{\nu}\right)$ in $\mathrm{eV}$ using various $\mathrm{CMB}$ experiments or combinations of them, and assuming that the lensing map is extracted following the quadratic estimator method. These numbers are all taken from Ref. [198], which assumes either an eight parameter model (the usual six of $\Lambda \mathrm{CDM}$ plus $M_{\nu}$ and $Y_{\mathrm{He}}$ ), or an eleven parameter model (the same ones plus $\alpha, w, N_{\text {eff }}$ ). The fiducial value of the total mass is taken to be $M_{\nu}=0.1 \mathrm{eV}$, and in each case the two numbers correspond to optimistic or pessimistic assumptions concerning the foregrounds contamination of the primary CMB signal.

\begin{tabular}{ccc} 
Experiment & 8 parameters & 11 parameters \\
\hline BICEP+QUaD & $0.31-0.36$ & $0.36-0.40$ \\
BRAIN+ClOVER & $0.34-0.43$ & $0.42-0.51$ \\
PLANCK & $0.13-0.14$ & $0.15-0.15$ \\
SAMPAN & $0.10-0.17$ & $0.12-0.18$ \\
PLANCK+SAMPAN & $0.08-0.10$ & $0.10-0.12$ \\
INFLATION PROBE & $0.032-0.036$ & $0.035-0.039$ \\
\hline
\end{tabular}

would be multiplied by three (by four in the case with extra free parameters). No significant difference between the forecast errors in the eight and eleven parameter models was found in Ref. [198]. SAMPAN alone is slightly more efficient than PLANCK, and the combination PLANCK+SAMPAN is the first one to reach $\sigma\left(M_{\nu}\right) \sim 0.1 \mathrm{eV}$, even in the pessimistic case of large foreground residuals and extra free parameters. Finally, the version of the INFLATiON Probe satellite considered in [198], which is slightly more ambitious than the CMBpol assumption of Ref. [197], is able to reach $\sigma\left(M_{\nu}\right) \sim 0.035 \mathrm{eV}$ both in the eight and eleven parameter cases. In any case, the results seem to be relatively robust against pessimistic assumptions concerning foreground contamination.

\subsection{Galaxy weak lensing (cosmic shear)}

\subsubsection{Principle}

Weak lensing changes the apparent shape of galaxies in various ways. Among others, it changes slightly their apparent ellipticity: i.e. galaxies which would be apparently spherical without weak lensing can look in fact elliptical, stretched in one direction and squeezed in the orthogonal direction. This effect, which is coherent over the angular size of the lensing gravitational field, i.e. potentially over many sources, is called cosmic shear. It can be detected provided one has a very dense sample of galaxies with enough resolution in order 
to measure each individual shape. In order to be able to reconstruct the lensing gravitational field, it is also necessary to know either the redshift of each source galaxy, or at least their coarse-grained number density $g(z, \hat{n})$ in redshift space and in direction $\hat{n}$. Let us assume for simplicity that we have an isotropic sample with a spherical number density $g(z)$ (or $g(r)$, where $r$ is the comoving radius). We can divide the sample in small solid angles or pixels. Since intrinsic ellipticities are randomly distributed, the average ellipticity in each pixel will give a measurement of the average cosmic shear, up to a shot noise term proportional to $N_{\text {pix }}^{-1 / 2}$, where $N_{\text {pix }}$ is the number of galaxies per pixel. Therefore, the size of the pixels must be chosen optimally in a trade-off between high angular resolution and high signal-to-noise.

The cosmic shear field reconstructed by this method derives from the same lensing potential $\phi$ as the deflection field discussed in the previous subsection. The relation between the lensing potential $\phi$ and the gravitational potential $\Phi$ along the line of sight is the same as in Eqs. (160) and (161), except that one must now average over all individual sources of comoving distance $r_{s} \in$ $\left[0, r_{s}^{\max }\right]$. In the continuous limit and for an ensemble of sources described by the density function $g(r)$, this gives

$$
\phi(\hat{n} ; g)=\frac{\int_{0}^{r_{s}^{\max }} d r_{s} g\left(r_{s}\right) \phi\left(\hat{n} ; r_{s}\right)}{\int_{0}^{r_{s}^{\max }} d r_{s} g\left(r_{s}\right)}=\int_{0}^{r_{s}^{\max }} d r W(r ; g) \Phi(r \hat{n}, \eta=-r),
$$

with

$$
W(r ; g) \equiv \frac{\int_{r}^{r_{s}^{\max }} d r_{s} g\left(r_{s}\right) W\left(r ; r_{s}\right)}{\int_{0}^{r_{s}^{\max }} d r_{s} g\left(r_{s}\right)}
$$

Like for CMB anisotropies, once we have the lensing potential map $\phi(\hat{n} ; g)$ we can compute the power spectrum $C_{l}^{\phi \phi, g}$, which gives us an information on the surrounding large scale structure projected along the line of sight. Therefore, cosmic shear surveys offer another opportunity to measure the twopoint correlation function of total matter fluctuations, complementary to the three-dimensional Fourier power spectrum $P(k)$ obtained from galaxy redshift surveys (probing lower redshifts) and to the CMB lensing power spectrum (probing higher redshifts).

If a shear survey contains a large enough number of galaxies with known 
redshifts, it is even possible to refine this technique by splitting the sources into redshift (or distance) bins, from $0<r_{s}<r_{1}$ to $r_{n-1}<r_{s}<r_{n}$. It is then possible to measure $n$ lensing potential maps $\phi\left(\hat{n}, g_{i}\right)$, such that the bin distribution $g_{i}\left(r_{s}\right)$ vanishes outside of the range $r_{i-1}<r_{s}<r_{i}$. From these maps one can obtain $n$ power spectra $C_{l}^{\phi \phi, g_{i}}$ and $n(n-1) / 2$ cross-correlation spectra $C_{l}^{\phi \phi, g_{i} g_{j}}$ (cross-correlations do not vanish because the same structure can contribute to the lensing of sources in different bins). Since the highest redshift bin probes structures at larger redshift, this method allows to some extent for a three-dimensional reconstruction of the gravitational fluctuations. This technique is called tomography, and brings some hope of tracking the evolution of $P(k, z)$ as a function of redshift caused by dark energy [233] and by neutrino masses (see Sec. 4.6.4).

\subsubsection{Neutrino mass from cosmic shear surveys}

There are various ongoing and planned cosmic shear surveys, like for instance the Canada-France-Hawaii Telescope Legacy Survey ${ }^{20}$ (CFHTLS) [162]; the Dark Energy Survey ${ }^{21}$ (DES) $[212,213,214]$; the SuperNova Acceleration Probe ${ }^{22}$ (SNAP); the Panoramic Survey Telescope And Rapid Response System ${ }^{23}$ (Pan-STARRS); or the Large Synoptic Survey Telescope ${ }^{24}$ (LSST) [234,235]. The sensitivity of this type of observation to neutrino masses has been addressed first in [236], and later in $[237,206,238]$. Here we will review the results of [206], where four hypothetical experiments are considered. The first two, called S300 and S1000, stand for future satellite surveys with an average source density of 100 galaxies per squared arc-minute and a sky coverage of 300 square degrees $\left(f_{\text {sky }}=0.0073\right)$ and 1000 squared degrees $\left(f_{\text {sky }}=0.024\right)$, respectively. The SNAP lensing survey could be close to one of these two assumptions. The last two, called G2 $\pi$ and G4 $\pi$, stand for future ground-based surveys with an average source density of 65 galaxies per square arc-minute and a sky coverage of respectively $f_{\text {sky }}=0.5$ (as expected for LSST) and $f_{\text {sky }}=1$ (as expected for Pan-STARRS). The authors make some realistic assumption concerning the redshift distribution of the sources (given experimental limitations) and perform a Fisher matrix analysis for the combination of the cosmic shear surveys with either PLANCK or CMBpol (see Table 4), including in each case information on the CMB lensing, extracted with the quadratic estimator method described in Sec. 6.4. They assume a tomographic analysis of the galaxy-ellipticity data with eight redshifts bins. Thanks to the quadratic estimator method, the CMB experiments also provide a measure-

\footnotetext{
$\overline{20}$ http: //www.cfht.hawaii.edu/Science/CFHLS/

${ }^{21}$ http://www. darkenergysurvey.org/

22 http://snap.lbl.gov

23 http://pan-starrs.ifa.hawaii.edu/

${ }^{24}$ http://www. lsst.org/lsst home.shtml
} 
Table 9

Forecast error $\sigma\left(M_{\nu}\right)$ in eV from reference [206], using some hypothetical cosmic shear surveys (S300, S1000, G2 $\pi$ and G4 $\pi$ ) described in the text, combined with either PLANCK or CMBpol (including, in each case, information on the CMB lensing, extracted with the quadratic estimator method).

\begin{tabular}{ccccc}
\hline & S300 & S1000 & G2 $\pi$ & G4 $\pi$ \\
\hline Planck & 0.12 & 0.090 & 0.052 & 0.045 \\
CMBpol & 0.047 & 0.045 & 0.031 & 0.027 \\
\hline
\end{tabular}

ment of the cosmic shear power spectrum, sensitive to higher redshifts: here the information from CMB lensing plays the role of a ninth redshift bin.

There are eleven free cosmological parameters in the analysis of Ref. [206]: the usual six $\Lambda \mathrm{CDM}$ parameters, plus the total neutrino mass (attributed to a single family), the primordial helium fraction, the running of the scalar tilt, and two parameters describing the dark energy equation of state: $w(a)=$ $w_{0}+\left(a_{0}-a\right) w_{a}$. The results are summarized in Table 9, from which one concludes that cosmic shear surveys constitute an extremely powerful tool for constraining the total neutrino mass. The sensitivities obtained with this method are the absolute best forecasts derived so far. This is not surprising, given the ideal properties of weak lensing observations: precise, high redshift measurement of the matter power spectrum over a wide range of scales, and tomography.

Following Ref. [206] and Table 9, the most ambitious hypothetical experiment G4 $\pi$ combined with the PLANCK data would reach $\sigma\left(M_{\nu}\right) \simeq 0.045 \mathrm{eV}$, i.e. the same sensitivity as CMBpol alone (see Sec. 6.4.3); while G4 $\pi$ combined with CMBpol would reach $\sigma\left(M_{\nu}\right) \simeq 0.027 \mathrm{eV}$, which means that a $2 \sigma$ detection of the neutrino mass would occur even for the smallest $M_{\nu}$ in the $\mathrm{NH}$ scheme, of order $\left(\Delta m_{\mathrm{atm}}^{2}\right)^{1 / 2} \simeq 0.05 \mathrm{eV}$. The results of Table 9 are relatively conservative in the sense that in the Fisher matrix computation, the cosmic shear power spectrum was limited to the angular scales $\theta>0.18 \mathrm{deg}$ (in a multipole expansion, $l<1000$ ) below which non-linearities render the power spectrum reconstruction more difficult. However, the authors of Ref. [206] refer to a method for extracting information on smaller scales proposed by Refs. $[239,240]$. Ref. [206] speculates that using this method, one could gain a factor three (two) with respect to the results of Table 9 for the combination of PLANCK (CMBpol) with G2 $\pi$ or G4 $\pi$. This would imply a real precision measurement of the total neutrino mass with cosmological observables.

Finally, the recent analysis in Ref. [238] considers the combination of PLANCK (no lensing extraction) with two lensing surveys inspired by the SNAP and LSST projects, using up to five tomography bins. The results are consistent with those of Ref. [206]. 


\subsection{Galaxy cluster surveys}

The last method that we will discuss is expected to bring some extra independent information, and could further improve the bounds discussed in the previous subsection.

In the past years, the study of the cluster abundance evolution (the function $N(z)$, where $N$ is the number density of galaxy clusters and $z$ the redshift) has lead to interesting constraints on the normalization of the matter power spectrum at small scales $\sigma_{8}$ (defined in Sec. 5.5), and on the matter density fraction $\Omega_{\mathrm{m}}$. For a given cosmological model, the function $N(z)$ can be predicted from non-linear simulations, and compared with experimental data from galaxy cluster surveys. The non-linear simulations generally introduce systematic errors, but since cluster scales are not too deep inside the non-linear regime even today, the method can be kept under control.

The function $N(z)$-or better, its derivative $d N / d z$ - is affected by the evolution of the linear growth factor on cluster scales. Therefore, as emphasized recently in [241], future precise measurements of cluster abundances will be useful probes of the neutrino free-streaming effect.

On the observational side, the prospects for galaxy cluster surveys are very promising, since catalogs will be derived from various techniques, by mapping the $\mathrm{X}$-ray emission of the hot cluster gas, or the scattering of CMB photons in this gas (Sunyaev-Zel'dovich effect, SZE), or the cosmic shear measured from galaxy weak lensing (as discussed in the previous subsection). We have already seen that ambitious projects are being planned for galaxy weak lensing; this is also the case for X-ray and SZE observations. The full data will then provide: first, a measurement of the matter power spectrum $P(k, z)$ (modulo some bias and redshift-space distortion effects), which can be measured in various redshift bins, like for weak lensing tomography; and second, an estimate of the abundance evolution $d N / d z$, also at various redshifts.

An expression of the Fisher matrix describing future $P(k, z)$ and $d N / d z(z)$ observations has been proposed in [242]; this forecast method was applied to neutrino mass extraction in [241], using the expected instrumental sensitivity of the following projects: for CMB temperature and polarization anisotropies, PlAnCK (no lensing extraction); for X-ray observations, the Dark Universe Observatory (DUO) survey (see [243] for the description of a similar, previously proposed survey), which should see $\sim 11,500$ clusters in 6,150 $\mathrm{deg}^{2}$; for SZE observations, the South Pole Telescope (SPT) survey [199], yielding $\sim 20,000$ clusters in 4,000 $\mathrm{deg}^{2}$; and for galaxy weak lensing, the LSST project (already introduced in Sec. 6.5.2), assuming $\sim 200,000$ clusters in 18,000 $\mathrm{deg}^{2}$. Among the three data sets, Ref. [241] finds that the most powerful should be 

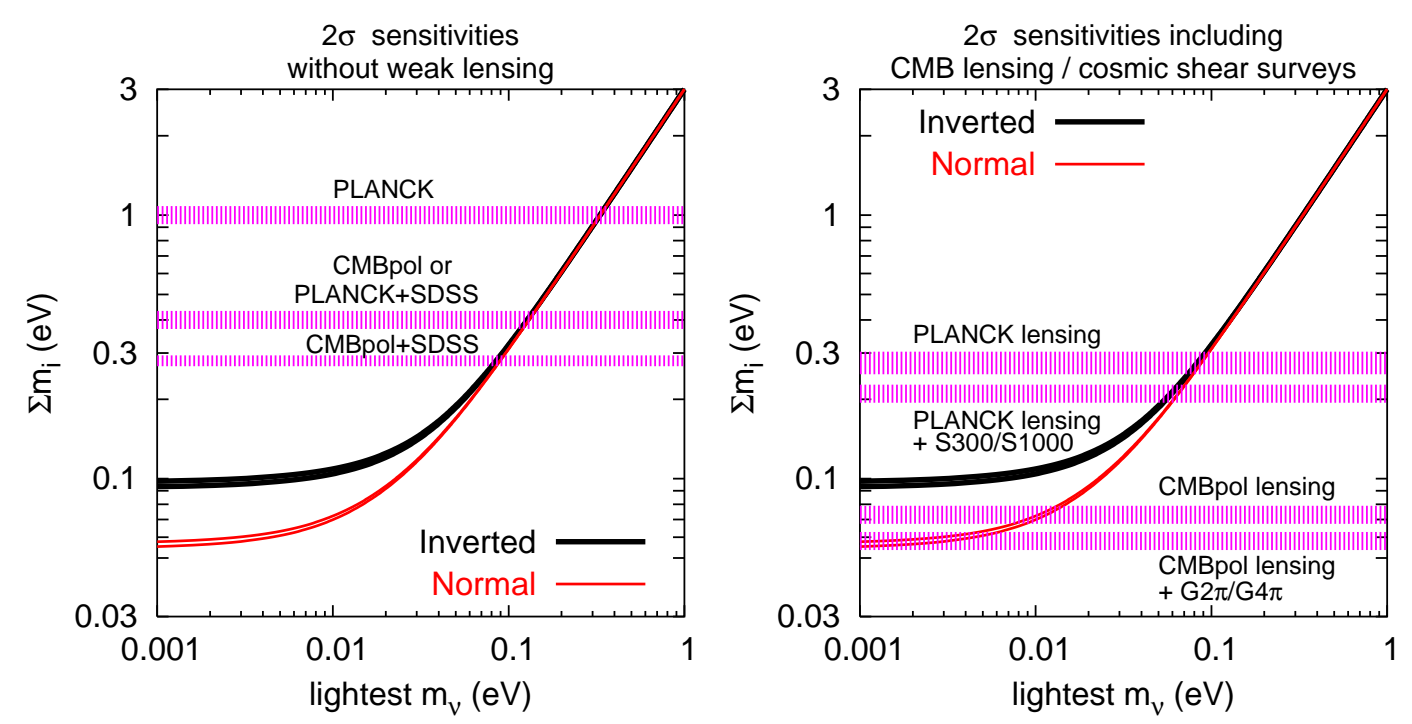

Fig. 23. Forecast $2 \sigma$ sensitivities to the total neutrino mass from future cosmological experiments, as described in Sec. 6, compared to the values in agreement with present neutrino oscillation data in Eq. (2) (assuming a future determination at the $5 \%$ level). Left: sensitivities expected for future CMB experiments (without lensing extraction), alone and combined with the completed SDSS galaxy redshift survey. Right: sensitivities expected for future CMB experiments including lensing information, alone and combined with future cosmic shear surveys. Here CMBpol refers to a hypothetical CMB experiment roughly corresponding to the INFLATION Probe mission.

the LSST lensing survey: the forecast error $\sigma\left(M_{\nu}\right)$ is typically $50 \%$ larger for SPT, and 60 to 100\% larger for DUO. For the combination PLANCK+LSST, it is interesting to note that the inclusion of $d N / d z$ improves the neutrino mass determination by a factor two: using only information from the CMB and from $P(k, z)$, the authors find $\sigma\left(M_{\nu}\right) \simeq 0.09 \mathrm{eV}$, while adding $d N / d z$ they get $\sigma\left(M_{\nu}\right) \simeq 0.04 \mathrm{eV}$. Including the DUO and SPT data marginally improves this bound.

\section{Conclusions}

Cosmology has played an important role in constraining neutrino properties, providing information on these elusive particles that complements the efforts of laboratory experiments, and neutrino cosmology will remain an active research field in the next years.

In this review, we were interested in the connection between neutrino masses and cosmology. We have described in detail how the evolution of cosmological perturbations is modified by massive neutrinos, and how their effects leave an imprint in the different cosmological observables. We focused on the standard 
case of three active neutrino species, known to be massive thanks to the experimental results on flavour neutrino oscillations which, however, do not fix the absolute neutrino mass scale.

We saw how the analysis of cosmological observables can provide either a positive indication or an upper bound on a total neutrino mass in the range from 0.05 to a few eV indicated by oscillation data and other laboratory results. We discussed why a unique cosmological bound does not exist: the values depend on the assumed cosmological model and the considered cosmological data. Within a minimal $\Lambda$ MDM model, the present cosmological bounds at 95\% CL are given in Tables 1-3, ranging from 2-3 eV with CMB data only to more stringent values when LSS data are added: 0.9-1.7 eV considering only the shape of the matter power spectrum from $2 \mathrm{dF}$ or SDSS data, or 0.3-0.9 $\mathrm{eV}$ fixing the light-to-mass bias and/or adding Lyman- $\alpha$ forest data and/or the SDSS measurement of the baryon oscillation peak. These values limit the neutrino masses in the so-called degenerate region, as presented in Fig. 19. We also discussed how these cosmological bounds could be relaxed or even disappear when adding some extra cosmological parameters or in scenarios with non-standard relic neutrinos. It is interesting to note that these mass bounds also apply for other thermal relic particles, such as axions with masses in the $\mathrm{eV}$ range as shown in [244].

Finally, we have discussed how the sensitivity to neutrino masses will improve with new cosmological data, such as new CMB experiments or larger galaxy redshift surveys. In particular we have reviewed the excellent prospects of future weak lensing measurements, either from CMB data or with cosmic shear surveys. We saw how these future data will be sensitive to a total neutrino mass well below $1 \mathrm{eV}$, even when considering extra cosmological parameters.

We give a graphical summary of the forecast sensitivities to neutrino masses of different cosmological data in Fig. 23, compared to the allowed values of neutrino masses in the two possible 3-neutrino schemes. One can see from this figure that there are very good prospects for testing neutrino masses in the degenerate and quasi-degenerate mass regions above $0.2 \mathrm{eV}$ or so. A detection at a significant level of the minimal value of the total neutrino mass in the inverted hierarchy scheme will demand the combination of future data from CMB lensing and cosmic shear surveys, whose more ambitious projects will provide a $2 \sigma$ sensitivity to the minimal value in the case of normal hierarchy (of order $0.05 \mathrm{eV}$ ). The combination of CMB observations with future galaxy cluster surveys (derived from the same weak lensing observations, as well as X-ray and Sunyaev-Zel'dovich surveys) should yield a similar sensitivity.

The information on neutrino masses from analyses of cosmological data is complementary and can not replace the efforts in terrestrial projects such as tritium beta decay and neutrinoless double beta decay experiments (and vice 
versa). In particular, a positive result from one of these three possibilities, which as reviewed in Sec. 2 do not measure the same combination of neutrino masses and mixing parameters, should be cross-checked by the others. Of course any information on the absolute neutrino mass scale will be a very important input for theoretical models of particle physics beyond the Standard Model.

Note that light massive neutrinos could also play a role in the generation of the baryon asymmetry of the Universe from a previously created lepton asymmetry. In these leptogenesis scenarios, one can also obtain quite restrictive bounds on light neutrino masses, which are however model-dependent (see [245] for a recent review).

This review was entirely devoted to the case of light neutrinos with masses of order eV or smaller. However, we would like to mention the case of a sterile neutrino with a mass of the order of a few keV's and a small mixing with the flavour neutrinos. It has been shown that these $\mathrm{keV}$ neutrinos could also have interesting cosmological consequences: they would play the role of dark matter and replace the usual CDM component (see [246,247,248,249] for an incomplete list of references). Because of their large thermal velocity (slightly smaller than that of active neutrinos), they would behave as Warm Dark Matter and erase small-scale cosmological structures. Their mass can be bounded from below using Lyman- $\alpha$ forest data from quasar spectra, and from above using X-ray observations [250,251,252,253,254]. This scenario, already disfavoured according to the results in [254], is currently under careful examination.

\section{Note added}

A few new analyses of cosmological data including non-zero neutrino masses appeared after this review was accepted for publication. In Ref. [255] the authors used data on the Lyman- $\alpha$ forest from SDSS (improved thanks to the inclusion of the mean flux constraints derived from a principal component analysis of quasar spectra), SDSS and 2dF galaxy clustering, SNIa and CMB power spectra (WMAP3 and others) to get an upper bound of $M_{\nu}<0.17 \mathrm{eV}$ (at $95 \% \mathrm{CL})$ on the total mass of the three active neutrinos $\left(M_{\nu}<0.26 \mathrm{eV}\right.$ for four thermalized species). Instead, in Ref. [256] the limit $M_{\nu}<2.0 \mathrm{eV}$ for three neutrinos was found using WMAP3 data only, in agreement with the result of the WMAP team [65]. 


\section{Acknowledgments}

We would like to thank Elena Pierpaoli for many useful comments, as well as Alessandro Cuoco, Alexander Dolgov, Kazuhide Ichikawa, Gianpiero Mangano, Laurence Perotto, Georg Raffelt, Rafael Rebolo, José A. Rubiño-Martín, Subir Sarkar and Pasquale Serpico for interesting feedback on this manuscript. This work was supported by a MEC-IN2P3 agreement and by the European Network of Theoretical Astroparticle Physics ILIAS/N6 under contract number RII3-CT-2004-506222. SP was supported by the Spanish grants FPA200501269, BFM2002-00345 and GV/05/017 of Generalitat Valenciana, as well as by a Ramón y Cajal contract of MEC.

\section{References}

[1] S. Hannestad, New J. Phys. 6 (2004) 108 hep-ph/0404239.

[2] Ø. Elgarøy and O. Lahav, New J. Phys. 7 (2005) 61 hep-ph/0412075.

[3] S. Hannestad, Prog. Part. Nucl. Phys. 57 (2006) 309 astro-ph/0511595.

[4] M. Tegmark, in Proceedings of Nobel Symposium 2004, Enköping, Sweden (August 2004), hep-ph/0503257.

[5] S. Hannestad, hep-ph/0602058.

[6] A.D. Dolgov, Phys. Rep. 370 (2002) 333 hep-ph/0202122.

[7] M. Kamionkowski and A. Kosowsky, Ann. Rev. Nucl. Part. Sci. 49 (1999) 77 astro-ph/9904108.

[8] M.C. González-García and Y. Nir, Rev. Mod. Phys. 75 (2003) 345 hep-ph/0202058.

[9] M. Hirsch and J.W.F. Valle, New J. Phys. 6 (2004) 76 hep-ph/0405015.

[10] G. Altarelli and F. Feruglio, New J. Phys. 6 (2004) 106 hep-ph/0405048.

[11] R.N. Mohapatra and A.Yu. Smirnov, hep-ph/0603118.

[12] S. Eidelman et al [Particle Data Group], Phys. Lett. B 592 (2004) 1.

[13] M. Maltoni, T. Schwetz, M.A. Tórtola and J.W.F. Valle, New J. Phys. 6 (2004) 122 hep-ph/0405172.

[14] G.L. Fogli, E. Lisi, A. Marrone and A. Palazzo, Prog. Part. Nucl. Phys., in press hep-ph/0506083.

[15] S.R. Elliott and P. Vogel, Ann. Rev. Nucl. Part. Sci. 52 (2002) 115 hep-ph/0202264. 
[16] S.M. Bilenky, C. Giunti, J.A. Grifols and E. Massó, Phys. Rep. 379 (2003) 69 hep-ph/0211462.

[17] V. Barger, D. Marfatia and K. Whisnant, Int. J. Mod. Phys. E 12 (2003) 569 hep-ph/0308123.

[18] R.D. McKeown and P. Vogel, Phys. Rep. 394 (2004) 315 hep-ph/0402025.

[19] A. Aguilar et al [LSND Coll.], Phys. Rev. D 64 (2001) 112007 hep-ex/0104049.

[20] A. Aguilar-Arevalo et al [MiniBooNE coll.], The MiniBooNE Run Plan (2003), available at http://www-boone.fnal.gov/publicpages/runplan.ps.gz

[21] K. Eitel, Nucl. Phys. Proc. Suppl. 143 (2005) 197.

[22] V.M. Lobashev, Nucl. Phys. A 719 (2003) 153.

[23] C. Kraus et al, Eur. Phys. J. C 40 (2005) 447 hep-ex/0412056.

[24] A. Osipowicz et al, hep-ex/0109033. KATRIN experiment homepage at http://www-ik.fzk.de/katrin/

[25] A. Strumia and F. Vissani, Nucl. Phys. B 726 (2005) 294 hep-ph/0503246.

[26] V.A. Rodin, A. Faessler, F. Šimkovic and P. Vogel, Nucl. Phys. A 766 (2006) 107 nucl-th/0503063.

[27] H.V. Klapdor-Kleingrothaus et al, Eur. Phys. J. A 12 (2001) 147 hep-ph/0103062.

[28] C.E. Aalseth et al [IGEX Coll.], Phys. Rev. C 59 (1999) 2108.

[29] C. Arnaboldi et al [Cuoricino Coll.], Phys. Rev. Lett. 95 (2005) 142501 hep-ex/0501034.

[30] R. Arnold et al [NEMO Coll.], Phys. Rev. Lett. 95 (2005) 182302 hep-ex/0507083.

[31] H.V. Klapdor-Kleingrothaus, I.V. Krivosheina, A. Dietz and O. Chkvorets, Phys. Lett. B 586 (2004) 198 hep-ph/0404088.

[32] S.T. Petcov, New J. Phys. 6 (2004) 109.

[33] S. Pascoli, S.T. Petcov and T. Schwetz, Nucl. Phys. B 734 (2006) 24 hep-ph/0505226.

[34] E. Nardi and J.I. Zuluaga, Nucl. Phys. B 731 (2005) 140 hep-ph/0412104.

[35] A.D. Dolgov et al, Nucl. Phys. B 632 (2002) 363 hep-ph/0201287.

[36] Y.Y.Y. Wong, Phys. Rev. D 66 (2002) 025015 hep-ph/0203180.

[37] K.N. Abazajian, J.F. Beacom and N.F. Bell, Phys. Rev. D 66 (2002) 013008 astro-ph/0203442. 
[38] P.D. Serpico and G.G. Raffelt, Phys. Rev. D $\quad 71 \quad$ (2005) 127301 astro-ph/0506162.

[39] V. Barger et al, Phys. Lett. B 569 (2003) 123 hep-ph/0306061.

[40] A. Cuoco et al, Int. J. Mod. Phys. A 19 (2004) 4431 astro-ph/0307213.

[41] E.W. Kolb and M.S. Turner, The Early Universe (Addison-Wesley, 1990).

[42] S. Dodelson, Modern Cosmology (Academic Press, 2003).

[43] S. Hannestad and J. Madsen, Phys. Rev. D 52 (1995) 1764 astro-ph/9506015.

[44] A.D. Dolgov, S.H. Hansen and D.V. Semikoz, Nucl. Phys. B 503 (1997) 426 hep-ph/9703315.

[45] S. Esposito et al, Nucl. Phys. B 590 (2000) 539 astro-ph/0005573.

[46] G. Mangano, G. Miele, S. Pastor and M. Peloso, Phys. Lett. B 534 (2002) 8 astro-ph/0111408.

[47] G. Mangano et al, Nucl. Phys. B 729 (2005) 221 hep-ph/0506164.

[48] S. Hannestad, Phys. Rev. D 65 (2002) 083006 astro-ph/0111423.

[49] U. Seljak and M. Zaldarriaga, Astrophys. J. 469 (1996) 437 astro-ph/9603033. See also the webpage http://cmbfast.org

[50] A. Lewis, A. Challinor and A. Lasenby, Astrophys. J. 538 (2000) 473 astro-ph/9911177. See also the webpage http://camb.info

[51] V.F. Shvartsman, Pisma Zh. Eksp. Teor. Fiz. 9 (1969) 315 [JETP Lett. 9 (1969) 184].

[52] G. Steigman, D.N. Schramm and J.R. Gunn, Phys. Lett. B 66 (1977) 202.

[53] S. Sarkar, Rep. Prog. Phys. 59 (1996) 1493 hep-ph/9602260.

[54] M. Cirelli, G. Marandella, A. Strumia and F. Vissani, Nucl. Phys. B 708 (2005) 215 hep-ph/0403158.

[55] D.N. Spergel et al [WMAP Coll.], Astrophys. J. Suppl. 148 (2003) 175 astro-ph/0302209.

[56] S. Hannestad, JCAP 0305 (2003) 004 astro-ph/0303076.

[57] V. Barger et al, Phys. Lett. B 566 (2003) 8 hep-ph/0305075.

[58] R.H. Cyburt, B.D. Fields, K.A. Olive and E. Skillman, Astropart. Phys. 23 (2005) 313 astro-ph/0408033.

[59] G. Steigman, Int. J. Mod. Phys. E 15 (2006) 1 astro-ph/0511534.

[60] K.A. Olive and E.D. Skillman, Astrophys. J. 617 (2004) 29 astro-ph/0405588.

[61] P.D. Serpico et al, JCAP 0412 (2004) 010 astro-ph/0408076. 
[62] P. Crotty, J. Lesgourgues and S. Pastor, Phys. Rev. D 67 (2003) 123005 astro-ph/0302337.

[63] E. Pierpaoli, Mon. Not. Roy. Astron. Soc. 342 (2003) L63 astro-ph/0302465.

[64] S. Hannestad, JCAP 0601 (2006) 001 astro-ph/0510582.

[65] D.N. Spergel et al [WMAP Coll.], astro-ph/0603449.

[66] A. Ringwald and Y.Y.Y. Wong, JCAP 0412 (2004) 005 hep-ph/0408241.

[67] S.S. Gershtein and Ya.B. Zeldovich, JETP Lett. 4 (1966) 120 [Pisma Zh. Eksp. Teor. Fiz. 4 (1966) 174].

[68] R. Cowsik and J. McClelland, Phys. Rev. Lett. 29 (1972) 669.

[69] J.R. Primack, SLAC Beam Line 31N3 (2001) 50 astro-ph/0112336.

[70] J.R. Bond, G. Efstathiou and J. Silk, Phys. Rev. Lett. 45 (1980) 1980.

[71] J.R. Bond and A.S. Szalay, Astrophys. J. 274 (1983) 443.

[72] S.D.M. White, C.S. Frenk and M. Davis, Astrophys. J. 274 (1983) L1.

[73] R. Valdarnini, T. Kahniashvili and B. Novosyadlyj, Astron. Astrophys. 336 (1998) 11 astro-ph/9804057.

[74] C.P. Ma and E. Bertschinger, Astrophys. J. 455 (1995) 7 astro-ph/9506072.

[75] W.T. Hu, Ph.D. thesis (Univ. of California at Berkeley, 1995), astro-ph/9508126.

[76] A.R. Liddle and D.H. Lyth, Cosmological inflation and large-scale structure (Cambridge Univ. Press, 2000).

[77] E. Bertschinger, Proceedings of Cosmology 2000, Lisbon, Portugal (July 2000), astro-ph/0101009.

[78] M. Bucher, K. Moodley and N. Turok, Phys. Rev. D 62 (2000) 083508 astro-ph/9904231.

[79] R. Trotta, A. Riazuelo and R. Durrer, Phys. Rev. Lett. 87 (2001) 231301 astro-ph/0104017.

[80] D.H. Lyth, C. Ungarelli and D. Wands, Phys. Rev. D 67, 023503 (2003) astro-ph/0208055.

[81] P. Crotty, J. García-Bellido, J. Lesgourgues and A. Riazuelo, Phys. Rev. Lett. 91 (2003) 171301 astro-ph/0306286.

[82] M. Bucher et al, Phys. Rev. Lett. 93 (2004) 081301 astro-ph/0401417.

[83] K. Moodley et al, Phys. Rev. D 70 (2004) 103520 astro-ph/0407304.

[84] M. Beltrán, J. García-Bellido, J. Lesgourgues and A. Riazuelo, Phys. Rev. D 70 (2004) 103530 astro-ph/0409326. 
[85] S. Dodelson, E. Gates and A. Stebbins, Astrophys. J. 467 (1996) 10 astro-ph/9509147.

[86] K. Ichikawa and T. Takahashi, astro-ph/0510849.

[87] L.A. Kofman, N.Y. Gnedin and N.A. Bahcall, Astrophys. J. 413 (1993) 1.

[88] J.M. Bardeen, J.R. Bond, N. Kaiser and A.S. Szalay, Astrophys. J. 304 (1986) 15.

[89] D.J. Eisenstein et al, Astrophys. J. 633 (2005) 560 astro-ph/0501171.

[90] M.Yu. Khlopov, Cosmoparticle physics (World Scientific, 1999)

[91] S. Bashinsky and U. Seljak, Phys. Rev. D 69 (2004) 083002 astro-ph/0310198.

[92] R. Trotta and A. Melchiorri, Phys. Rev. Lett. 95 (2005) 011305 astro-ph/0412066.

[93] W. Hu and D.J. Eisenstein, Astrophys. J. 498 (1998) 497 astro-ph/9710216.

[94] W. Hu, D.J. Eisenstein and M. Tegmark, Phys. Rev. Lett. 80 (1998) 5255 astro-ph/9712057.

[95] J.A. Holtzman, Astrophys. J. Suppl. 71 (1989) 1.

[96] D.Y. Pogosyan and A.A. Starobinsky, Astrophys. J. 447 (1995) 465 astro-ph/9409074.

[97] C.P. Ma, Astrophys. J. 471 (1996) 13 astro-ph/9605198.

[98] D.J. Eisenstein and W. Hu, Astrophys. J. 511 (1997) 5 astro-ph/9710252.

[99] B. Novosyadlyj, R. Durrer and V.N. Lukash, Astron. Astrophys. 347 (1999) 799 astro-ph/9811262.

[100] P. Crotty, J. Lesgourgues and S. Pastor, Phys. Rev. D 69 (2004) 123007 hep-ph/0402049.

[101] J. Lesgourgues, S. Pastor and L. Perotto, Phys. Rev. D 70 (2004) 045016 hep-ph/0403296.

[102] M. Takada, E. Komatsu and T. Futamase, Phys. Rev. D 73 (2006) 083520 astro-ph/0512374.

[103] A. Slosar, astro-ph/0602133.

[104] R. Trotta, A. Riazuelo and R. Durrer, Phys. Rev. D 67 (2003) 063520 astro-ph/0211600.

[105] A. Upadhye, M. Ishak and P.J. Steinhardt, Phys. Rev. D 72 (2005) 063501 astro-ph/0411803.

[106] C. Yèche et al, Astron. Astrophys. 448 (2006) 831 astro-ph/0507170.

[107] G. Zech, Eur. Phys. J. direct C 4 (2002) 12 hep-ex/0106023. 
[108] R. Trotta, Ph.D. thesis (Univ. of Geneva, 2004), astro-ph/0410115.

[109] W.L. Freedman et al, Astrophys. J. 553 (2001) 47 astro-ph/0012376.

[110] M. Beltrán et al, Phys. Rev. D 71 (2005) 063532 astro-ph/0501477.

[111] R. Trotta, astro-ph/0504022.

[112] G. Hinshaw et al, Astrophys. J. Suppl. 148 (2003) 135 astro-ph/0302217.

[113] A. Kogut et al, Astrophys. J. Suppl. 148 (2003) 161 astro-ph/0302213.

[114] G. Hinshaw et al [WMAP Coll.], astro-ph/0603451.

[115] L. Page et al [WMAP Coll.], astro-ph/0603450.

[116] C.L. Kuo et al [ACBAR coll.], Astrophys. J. 600 (2004) 32 astro-ph/0212289.

[117] C. Dickinson et al, Mon. Not. Roy. Astron. Soc. 353 (2004) 732 astro-ph/0402498.

[118] A.C.S. Readhead et al, Astrophys. J. 609 (2004) 498 astro-ph/0402359.

[119] N.W. Halverson et al, Astrophys. J. 568 (2002) 38 astro-ph/0104489.

[120] M. Tristram et al, Astron. Astrophys. 436 (2005) 785 astro-ph/0411633.

[121] W.C. Jones et al, astro-ph/0507494.

[122] F. Piacentini et al, astro-ph/0507507.

[123] T.E. Montroy et al, astro-ph/0507514.

[124] S. Hanany et al, Astrophys. J. 545 (2000) L5 astro-ph/0005123.

[125] M. Tegmark et al, Phys. Rev. D 69 (2004) 103501 astro-ph/0310723.

[126] Ø. Elgarøy and O. Lahav, JCAP 0304 (2003) 004 astro-ph/0303089.

[127] K. Ichikawa, M. Fukugita and M. Kawasaki, Phys. Rev. D 71 (2005) 043001 astro-ph/0409768.

[128] M. Fukugita, contribution to the proceedings of NuFact'05, Frascati (Italy), June 2005 hep-ph/0511068

[129] A.G. Sánchez et al, Mon. Not. Roy. Astron. Soc. 366 (2006) 189 astro-ph/0507583.

[130] R. Rebolo and J.A. Rubiño-Martín, private communication.

[131] C.J. MacTavish et al, astro-ph/0507503.

[132] R. Rebolo et al, Mon. Not. Roy. Astron. Soc. 353 (2004) 747 astro-ph/0402466.

[133] W.J. Percival et al [The 2dFGRS Coll.], Mon. Not. Roy. Astron. Soc. 327 (2001) 1297 astro-ph/0105252. 
[134] S. Cole et al [The 2dFGRS Coll.], Mon. Not. Roy. Astron. Soc. 362 (2005) 505 astro-ph/0501174.

[135] M. Tegmark et al [SDSS Coll.], Astrophys. J. $606 \quad$ (2004) 702 astro-ph/0310725.

[136] L. Verde et al, Astrophys. J. Suppl. 148 (2003) 195 astro-ph/0302218.

[137] U. Seljak et al, Phys. Rev. D 71 (2005) 103515 astro-ph/0407372.

[138] U. Seljak et al, Phys. Rev. D 71 (2005) 043511 astro-ph/0406594.

[139] V. Barger, D. Marfatia and A. Tregre, Phys. Lett. B595 (2004) 55 hep-ph/0312065.

[140] G.L. Fogli et al, Phys. Rev. D 70 (2004) 113003 hep-ph/0408045.

[141] R.E. Smith et al [The Virgo Consortium Coll.], Mon. Not. Roy. Astron. Soc. 341 (2003) 1311 astro-ph/0207664.

[142] X. Wang, M. Tegmark and M. Zaldarriaga, Phys. Rev. D 65 (2002) 123001 astro-ph/0105091.

[143] W. Saunders et al, Mon. Not. Roy. Astron. Soc. 317 (2000) 55 astro-ph/0001117.

[144] Ø. Elgarøy et al, Phys. Rev. Lett. 89 (2002) 061301 astro-ph/0204152.

[145] S. Perlmutter et al, Astrophys. J. 517 (1999) 565 astro-ph/9812133.

[146] A.G. Riess et al, Astrophys. J. 607 (2004) 665 astro-ph/0402512.

[147] J.L. Tonry et al, Astrophys. J. 594 (2003) 1 astro-ph/0305008.

[148] P. Astier et al, Astron. Astrophys. 447 (2006) 31 astro-ph/0510447.

[149] R.A.C. Croft et al, Astrophys. J. 581 (2002) 20 astro-ph/0012324.

[150] N.Y. Gnedin and A.J.S. Hamilton, Mon. Not. Roy. Astron. Soc. 334 (2002) 107 astro-ph/0111194.

[151] S. Hannestad, in Proceedings of SEESAW25, Paris, France (June 2004) hep-ph/0409108.

[152] A. Goobar, S. Hannestad, E. Mortsell and H. Tu, astro-ph/0602155

[153] H. Bi, Astrophys. J. 405 (1993) 479.

[154] M. Viel et al, Mon. Not. Roy. Astron. Soc. 329 (2002) 848 astro-ph/0105233.

[155] M. Zaldarriaga, R. Scoccimarro and L. Hui, Astrophys. J. 590 (2003) 1 astro-ph/0111230.

[156] L. Hui, N.Y. Gnedin and Y. Zhang, Astrophys. J. 486 (1997) 599 astro-ph/9608157. 
[157] R.A.C. Croft, W. Hu and R. Davé, Phys. Rev. Lett. 83 (1999) 1092 astro-ph/9903335.

[158] M. Viel, M.G. Haehnelt and V. Springel, Mon. Not. Roy. Astron. Soc. 354 (2004) 684 astro-ph/0404600.

[159] P. McDonald et al, Astrophys. J. Suppl. 163 (2006) 80 astro-ph/0405013.

[160] P. McDonald et al, Astrophys. J. 635 (2005) 761 astro-ph/0407377.

[161] E. Pierpaoli, S. Borgani, D. Scott and M.J. White, Mon. Not. Roy. Astron. Soc. 342 (2003) 163 astro-ph/0210567.

[162] E. Semboloni et al, astro-ph/0511090.

[163] E. Pierpaoli, Proceedings of the "Multiwavelength Cosmology" conference, Mykonos Island, Greece (June 2003). Ed. by M. Plionis (Kluwer Academic Pub., 2004) p. 93

[164] M. Fukugita, G.C. Liu and N. Sugiyama, Phys. Rev. Lett. 84 (2000) 1082 hep-ph/9908450.

[165] T. Kahniashvili, E. von Toerne, N.A. Arhipova and B. Ratra, Phys. Rev. D 71 (2005) 125009 astro-ph/0503328.

[166] S.W. Allen, R.W. Schmidt and S.L. Bridle, Mon. Not. Roy. Astron. Soc. 346 (2003) 593 astro-ph/0306386.

[167] S. Hannestad and G. Raffelt, JCAP 0404 (2004) 008 hep-ph/0312154.

[168] M. Lattanzi, R. Ruffini and G.V. Vereshchagin, Phys. Rev. D 72 (2005) 063003 astro-ph/0509079.

[169] S. Dodelson, A. Melchiorri and A. Slosar, astro-ph/0511500.

[170] S. Hannestad, Phys. Rev. Lett. 95 (2005) 221301 astro-ph/0505551.

[171] A. Blanchard, M. Douspis, M. Rowan-Robinson and S. Sarkar, Astron. Astrophys. 412 (2003) 35 astro-ph/0304237.

[172] A.D. Dolgov and A.Yu. Smirnov, Phys. Lett. B 621 (2005) 1 hep-ph/0501066.

[173] A.D. Dolgov, S.H. Hansen and A.Yu. Smirnov, JCAP 0506 (2005) 004 astro-ph/0503612.

[174] M. Kawasaki, K. Kohri and N. Sugiyama, Phys. Rev. Lett. 82 (1999) 4168 astro-ph/9811437.

[175] M. Kawasaki, K. Kohri and N. Sugiyama, Phys. Rev. D 62 (2000) 023506 astro-ph/0002127.

[176] G.F. Giudice et al, Phys. Rev. D 64 (2001) 043512 hep-ph/0012317.

[177] P. Adhya, D.R. Chaudhuri and S. Hannestad, Phys. Rev. D 68 (2003) 083519 astro-ph/0309135. 
[178] S. Hannestad, Phys. Rev. D 70 (2004) 043506 astro-ph/0403291.

[179] K. Ichikawa, M. Kawasaki and F. Takahashi, Phys. Rev. D 72 (2005) 043522 astro-ph/0505395.

[180] S. Hannestad, Phys. Rev. D 59 (1999) 125020 astro-ph/9903475.

[181] M. Kaplinghat, R.E. Lopez, S. Dodelson and R.J. Scherrer, Phys. Rev. D 60 (1999) 123508 astro-ph/9907388.

[182] D. Kirilova, Int. J. Mod. Phys. D 13 (2004) 831 hep-ph/0209104.

[183] K. Abazajian, N.F. Bell, G.M. Fuller and Y.Y.Y. Wong, Phys. Rev. D 72 (2005) 063004 astro-ph/0410175.

[184] A. Cuoco, J. Lesgourgues, G. Mangano and S. Pastor, Phys. Rev. D 71 (2005) 123501 astro-ph/0502465.

[185] J.F. Beacom, N.F. Bell and S. Dodelson, Phys. Rev. Lett. 93 (2004) 121302 astro-ph/0404585.

[186] S. Hannestad, JCAP 0502 (2005) 011 astro-ph/0411475.

[187] S. Hannestad and G. Raffelt, Phys. Rev. D 72 (2005) 103514 hep-ph/0509278.

[188] N.F. Bell, E. Pierpaoli and K. Sigurdson, Phys. Rev. D 73 (2006) 063523 astro-ph/0511410.

[189] P.Q. Hung, hep-ph/0010126.

[190] R. Fardon, A.E. Nelson and N. Weiner, JCAP 0410 (2004) 005 astro-ph/0309800.

[191] E.J. Copeland, M. Sami and S. Tsujikawa, hep-th/0603057.

[192] A.W. Brookfield, C. van de Bruck, D.F. Mota and D. Tocchini-Valentini, Phys. Rev. D 73 (2006) 083515 astro-ph/0512367.

[193] M. Tegmark, A. Taylor and A. Heavens, Astrophys. J. 480 (1997) 22 astro-ph/9603021.

[194] M. Tegmark, Phys. Rev. D 56 (1997) 4514 astro-ph/9705188.

[195] G. Jungman, M. Kamionkowski, A. Kosowsky and D.N. Spergel, Phys. Rev. Lett. 76 (1996) 1007 astro-ph/9507080.

[196] D.J. Eisenstein, W. Hu and M. Tegmark, Astrophys. J. 518 (1998) 2 astro-ph/9807130.

[197] M. Kaplinghat, L. Knox and Y.S. Song, Phys. Rev. Lett. 91 (2003) 241301 astro-ph/0303344.

[198] J. Lesgourgues, L. Perotto, S. Pastor and M. Piat, Phys. Rev. D 73 (2006) 045021 astro-ph/0511735. 
[199] J. Ruhl et al [SPT Coll.], in Astronomical Structures and Mechanisms Technology, ed. by J. Antebi and D. Lemke, proceedings of the SPIE 5498 (2004) 11.

[200] B.G. Keating et al, in Polarimetry in Astronomy, ed. by S. Fineschi, Proceedings of the SPIE 4843 (2003) 284.

[201] S. Church et al [QUaD Coll.], New Astron. Rev. 47 (2003) 1083.

[202] M. Piat et al [BRAIN-CLOVER Coll.], SF2A 2004 proceedings (2004) astro-ph/0412590.

[203] B. Maffei et al [BRAIN-ClOVER Coll.], EAS Publications Series 14 (2005) 251.

[204] J.A. Tauber, Adv. Space Res. 34 (2004) 491.

[205] F.R. Bouchet et al, SF2A 2005 proceedings (2005) astro-ph/0510423.

[206] Y.S. Song and L. Knox, Phys. Rev. D 70 (2004) 063510 astro-ph/0312175.

[207] S. Hannestad, Phys. Rev. D 67 (2003) 085017 astro-ph/0211106.

[208] J. Lesgourgues, S. Pastor and S. Prunet, Phys. Rev. D 62 (2000) 023001 hep-ph/9912363.

[209] L. Popa, C. Burigana, F. Finelli and N. Mandolesi, Astron. Astrophys. 363 (2000) 825 astro-ph/0009417.

[210] M. Tegmark, Phys. Rev. Lett. 79 (1997) 3806 astro-ph/9706198.

[211] M. Moles et al, astro-ph/0504545.

[212] B. Flaugher [Dark Energy Survey Coll.], Int. J. Mod. Phys. A 20 (2005) 3121.

[213] J. Annis et al, astro-ph/0510195.

[214] T. Abbott et al [Dark Energy Survey Coll.], astro-ph/0510346.

[215] P. Fosalba, E. Gaztañaga and F. Castander, Astrophys. J. 597 (2003) L89 astro-ph/0307249.

[216] R. Scranton et al [SDSS Coll.], astro-ph/0307335.

[217] F. Bernardeau, Astron. Astrophys. 324 (1997) 15. astro-ph/9611012.

[218] M. Zaldarriaga and U. Seljak, Phys. Rev. D $58 \quad$ (1998) 023003 astro-ph/9803150.

[219] U. Seljak and M. Zaldarriaga, Phys. Rev. Lett. 82 (1999) 2636 astro-ph/9810092.

[220] A. Lewis and A. Challinor, Phys. Rep. 429 (2006) 1 astro-ph/0601594.

[221] C.M. Hirata and U. Seljak, Phys. Rev. D 68 (2003) 083002 astro-ph/0306354. 
[222] A. Cooray, M. Kamionkowski and R.R. Caldwell, Phys. Rev. D 71 (2005) 123527 astro-ph/0503002.

[223] W. Hu, Astrophys. J. 557 (2001) L79 astro-ph/0105424.

[224] W. Hu and T. Okamoto, Astrophys. J. 574 (2002) 566 astro-ph/0111606.

[225] T. Okamoto and W. Hu, Phys. Rev. D 67 (2003) 083002 astro-ph/0301031

[226] A. Amblard, C. Vale and M.J. White, New Astron. 9 (2004) 687 astro-ph/0403075.

[227] J. Lesgourgues, M. Liguori, S. Matarrese and A. Riotto, Phys. Rev. D 71 (2005) 103514 astro-ph/0412551.

[228] N. Bartolo, E. Komatsu, S. Matarrese and A. Riotto, Phys. Rep. 402 (2004) 103 astro-ph/0406398.

[229] U. Seljak, Astrophys. J. 463 (1996) 1 astro-ph/9505109.

[230] A. Cooray and M. Kesden, New Astron. 8 (2003) 231 astro-ph/0204068.

[231] K.M. Smith, W. Hu and M. Kaplinghat, Phys. Rev. D 70 (2004) 043002 astro-ph/0402442.

[232] W. Hu, Phys. Rev. D 64 (2001) 083005 astro-ph/0105117.

[233] W. Hu, Astrophys. J. 522 (1999) L21 astro-ph/9904153.

[234] J.A. Tyson, D.M. Wittman, J.F. Hennawi and D.N. Spergel, Nucl. Phys. Proc. Suppl. 124 (2003) 21 astro-ph/0209632.

[235] J.A. Tyson [the LSST Coll.], Proc. SPIE Int. Soc. Opt. Eng. 4836 (2002) 10 astro-ph/0302102.

[236] A.R. Cooray, Astron. Astrophys. 348 (1999) 31 astro-ph/9904246.

[237] K.N. Abazajian and S. Dodelson, Phys. Rev. Lett. 91 (2003) 041301 astro-ph/0212216.

[238] S. Hannestad, H. Tu and Y.Y.Y. Wong, astro-ph/0603019.

[239] B. Jain and A. Taylor, Phys. Rev. Lett. 91 (2003) 141302 astro-ph/0306046.

[240] G.M. Bernstein and B. Jain, Astrophys. J. 600 (2004) 17 astro-ph/0309332.

[241] S. Wang et al, Phys. Rev. Lett. 95 (2005) 011302 astro-ph/0505390.

[242] S. Wang, J. Khoury, Z. Haiman and M. May, Phys. Rev. D 70 (2004) 123008 astro-ph/0406331.

[243] K. Jahoda [The DUET Coll.], Astron. Nachr. $324 \quad$ (2003) 132 astro-ph/0211287

[244] S. Hannestad, A. Mirizzi and G. Raffelt, JCAP 0507 (2005) 002 hep-ph/0504059. 
[245] W. Buchmüller, P. Di Bari and M. Plümacher, Annals Phys. 315 (2005) 305 hep-ph/0401240.

[246] S. Dodelson and L.M. Widrow, Phys. Rev. Lett. 72 (1994) 17 hep-ph/9303287.

[247] A.D. Dolgov and S.H. Hansen, Astropart. Phys. 16, 339 (2002) hep-ph/0009083.

[248] K. Abazajian, Phys. Rev. D 73, 063506 (2006) astro-ph/0511630.

[249] A. Boyarsky et al, astro-ph/0603660

[250] K. Abazajian, G.M. Fuller and W.H. Tucker, Astrophys. J. 562 (2001) 593 astro-ph/0106002.

[251] S.H. Hansen, J. Lesgourgues, S. Pastor and J. Silk, Mon. Not. Roy. Astron. Soc. 333, 544 (2002) astro-ph/0106108.

[252] M. Viel et al, Phys. Rev. D 71, 063534 (2005) astro-ph/0501562.

[253] K. Abazajian, Phys. Rev. D 73 (2006) 063513 astro-ph/0512631.

[254] U. Seljak, A. Makarov, P. McDonald and H. Trac, astro-ph/0602430.

[255] U. Seljak, A. Slosar and P. McDonald, astro-ph/0604335.

[256] M. Fukugita, K. Ichikawa, M. Kawasaki and O. Lahav, astro-ph/0605362. 